\title{
Comparison of the Role of Beamwidth in Biological and Engineered Sonar
}

\author{
Bryan D. Todd \\ Thesis submitted to the Faculty of the \\ Virginia Polytechnic Institute and State University \\ in partial fulfillment of the requirements for the degree \\ Master of Science \\ in \\ Mechanical Engineering \\ Rolf Müller, Chair \\ Alexander Leonessa \\ Pinhas Ben-Tzvi
}

September 29, 2017

Blacksburg, Virginia

Keywords: Bat, Beamwidth, Bioinspired, Sonar

Copyright 2017, Bryan D. Todd 


\title{
Comparison of the Role of Beamwidth in Biological and Engineered Sonar
}

\author{
Bryan D. Todd
}

\author{
(ABSTRACT)
}

Sonar is an important sensory modality for engineers as well as in nature. In engineering, sonar is the dominating modality for underwater sensing. In biology, it is likely to have been a central factor behind the unprecedented evolutionary success of bats, a highly diverse group that accounts for over $20 \%$ of all mammal species. However, it remains unclear to what extent engineered and biosonar follow similar design and operational principles. In the current work, the key sonar design characteristic of beamwidth is examined in technical and biosonar. To this end, beamwidth data has been obtained for 23 engineered sonar systems and from numerical beampattern predictions for 151 emission and reception elements (noseleaves and pinnae) from bat biosonar. Beamwidth data from these sources is compared to the beamwidth of a planar ellipsoidal transducer as a reference. The results show that engineered and biological both obey the basic physical limit on beamwidth as a function of the ratio of aperture size and wavelength. However, beyond that, the beamwidth data revealed very different behaviors between the engineered and the biological sonar systems. Whereas the beamwidths of the technical sonar systems were very close to the planar transducer limit, the biological samples showed a very wide scatter away from this limit. This scatter was as large - if not wider - than what was seen in a small reference data set obtained with random aluminum cones. A possible interpretation of these differences in the variability could be that whereas sonar engineers try to minimize beamwidth subject to constraints on device size, the evolutionary optimization of bat biosonar beampatterns has been directed at other factors that have left beamwidth as a byproduct. Alternatively, the biosonar systems may require beamwidth values that are larger than the physical limit and differ between species and their sensory ecological niches.

This work was supported by grants from the National Science Foundation (NSF Grant No. ID 1362886) and the Naval Engineering Education Consortium (NEEC, contract number N00174-16-C-0026). 


\title{
Comparison of the Role of Beamwidth in Biological and Engineered Sonar
}

\author{
Bryan D. Todd
}

(GENERAL AUDIENCE ABSTRACT)

Sonar is an important method of sensing for engineers in undersea environments, but it is also used by several species of animals for for everyday use. The most prominent species that uses sonar, or echolocation, are bats, one of the most diverse groups of mammals. The study of bat biosonar systems serves as a counterpoint to many of the concepts in technical sonar. In technical sonar, arrays are made to be larger in size, with more elements, and operate at higher frequencies in order to decrease their beamwidth which increases their resolution. Unlike technical sonars bats must rely on smaller sized systems that they can carry around and they operate in air which has worse qualities for propagating sound waves. Even with these disadvantages, bats are able to operate in complex environments, such as dense vegetation, with ease. This work compared 151 emission and reception elements of bat biosonar systems with 23 engineered sonars to find that the biosonar had very different behavior from the engineered sonars. The engineered sonars, as well as a set of experimental baffles, closely followed the curve for the beamwidth limit of planar transducers but the biosonar samples had a large scatter from the curve. These results could be interpreted to show that while the engineered sonars attempt to minimize the beamwidth in order to maximize the resolution, the biosonar did not place much importance on having low beamwidths and high resolutions during its evolution. Alternatively, the results could indicate that it is preferable for biosonar to have larger beamwidths, a contrast to standard sonar design. 


\section{Acknowledgements}

I would like to thank Dr. Rolf Müller for having provided me with the opportunity to perform this research and teaching me so much along the way. I would also like to thank my committee members, Dr. Alexander Leonessa and Dr. Pinhas Ben-Tzvi. Additionally, I would like to thank Dr. Jason Gaudette from the Naval Undersea Warfare Center for the original idea of comparing technical and bat biosonar beamwidths and the Naval Engineering Education Consortium for sponsoring my research. 


\section{Contents}

1 Introduction 1

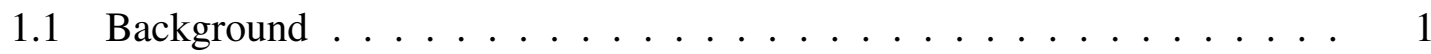

1.2 Review of Literature . . . . . . . . . . . . . . . 3

1.2.1 Bioinspiration and Biomimetics ............ 3

1.2.2 Bats and Biosonar .................. 4

1.2.3 Review of Technical Sonar Literature . . . . . . . . . . . . 5

1.2.4 Sonar Concepts . . . . . . . . . . . . . . . 6

1.2.5 Trends in Sonar Research . . . . . . . . . . . . 6

1.2.6 Conclusion .................... 7

1.3 Problem Statement and Research Objectives . . . . . . . . . . . . 8

1.3.1 The Case for Studying Biosonar . . . . . . . . . . . . . 8

1.3.2 Research Objectives and Hypotheses . . . . . . . . . . 8

2 Methods 11

2.1 Biosonar Apertures and Conversion to Engineering Analogues . . . . . . 11

2.1.1 Biosonar Aperture Data . . . . . . . . . . . . 11

2.1.2 Conversion from $\mu \mathrm{CT}$ Scans to Engineering Analogue . . . . . . 12

2.2 Beamwidth Determination for Biosonar Apertures . . . . . . . . . . . 14

2.2.1 Importance of Beamwidth . . . . . . . . . . . . . . . 14 
2.2.2 Planar Array Reference Beamwidth . . . . . . . . . . . . . . 14

2.2.3 Generation of Numerically Derived Beampatterns . . . . . . . . . 15

2.2.4 Biosonar Beamwidth Evaluation . . . . . . . . . . . 15

2.2.5 Half-Power vs Quarter-Power Beamwidths . . . . . . . . . . . 16

2.3 Acquisition of Technical Sonar Data . . . . . . . . . . . . . 17

2.3.1 Military Sonar vs Commercial Sonar _. . . . . . . . . . . 17

2.3.2 Technical Sonars Used for Comparison . . . . . . . . . . . 18

2.4 Aluminum Random Emission Baffle Experiment . . . . . . . . . . . 18

2.4.1 Equipment and Setup . . . . . . . . . . . . . . . . 19

2.4.2 Creation of Random Emission Baffles . . . . . . . . . . . . . 19

3 Results $\quad 21$

3.1 Half-Power Beamwidths and Quarter-Power Beamwidths . . . . . . . 21

3.2 Beamwidth Comparison of Engineered and Biosonar . . . . . . . . . . . 24

3.3 Noseleaf Simulate Experiment Results Compared to Biosonar . . . . . 28

3.4 Comparison of Bat Families With and Without Active Biosonar . . . . . . 31

3.5 Emission vs Reception Elements . . . . . . . . . . . . . . 32

3.6 Additional Supporting Analysis _. . . . . . . . . . . . . 35

3.6.1 Maximum vs Minimum Beamwidth . . . . . . . . . . . 35

3.6.2 Geometric Effects on Beamwidth . . . . . . . . . . . 37

3.6.3 Distance To Limit . . . . . . . . . . . . . . . . . . . . . 38

4 Discussion $\quad 43$

4.1 Importance of Beamwidth in Biosonar . . . . . . . . . . . . . . . . 43

4.2 Differences in Emission and Reception . . . . . . . . . . . . . 44

5 Conclusion $\quad 46$ 
5.1 Summary .............................. 46

5.2 Conclusion . . . . . . . . . . . . . . . . 47

5.3 Importance of Results . . . . . . . . . . . . . . . . . . . . 48

5.4 Achievements . . . . . . . . . . . . . . . . . . 49

Bibliography $\quad \mathbf{5 0}$

$\begin{array}{ll}\text { Appendix } & 54\end{array}$

Appendix A: Code ......................... 54

Appendix A.1: Geometric Processing Code . . . . . . . . . . . 54

Appendix A.2: Beampattern Processing Code . . . . . . . . . . 65

Appendix A.3: Beampattern Post Processing Code . . . . . . . . . . 78

Appendix B: Raw Data Table . . . . . . . . . . . . . . . . . . . . 88 


\section{List of Figures}

1.1 A look at an engineered sonar system vs biosonar elements. A) An Edgetech 4200 Side Scan sonar system that contains sensors along it's 125 cm length [5] (Picture Copyright Edgetech, reproduced with permission) and B) A bat from the Rhinolophidae family with its right pinnae (outer ear) and noseleaf circled in purple. . . . . . . . . . . . . . .

1.2 Visual representation of the first hypothesis tested in the current work: A) shows the first form of the hypothesis, that the engineered sonars, represented by black triangles, and biosonars, grey dots, have similar scatter and closeness to the reference limit represented by the black line. B) shows the alternative hypothesis that the biosonar has a larger scatter. . . . . . . . .

1.3 Visual representation of the second hypothesis to be tested in the current work: A) shows the first form of the hypothesis, that the aluminum baffles, represented by black triangles, and biosonars, grey dots, have similar scatter and closeness to the reference limit represented by the black line. B) shows the alternative hypothesis that either the reference baffles or biosonar has less scatter. . . . . . . . . . . . . . .

2.1 Approximation of the biological shape samples by a planar elliptical transducer: A) $\mu \mathrm{CT}$ model of a noseleaf from a Sulawesi horseshoe bat (Rhinolophus celebensis), B) Point cloud resulting from detecting edge vertices in the mesh, C) Edge vertices projected into the fitting plane with lines along the minimum and maximum aperture lengths (dashed lines). . 
2.2 Conversion of two-dimensional, numerical beampattern estimates into onedimensional beampattern functions: A) a numerical beampattern estimate derived from the Sulawesi horseshoe bat (Rhinolophus celebensis) shown in Figure 2.1. B) One of the beampattern slices obtained at different rotation angles, shown in polar coordinates. C) The same beampattern slice shown in B) converted in to Cartesian coordinates. The horizontal lines indicate the $-3 \mathrm{~dB}$ and $-6 \mathrm{~dB}$ threshold levels . . . . . . . . . . . .

2.3 Setup for the experiment with the random aluminum baffles: A) Diagram of the experimental setup with dimensions of the loudspeaker, waveguide (shown in blue), and random aluminum foil baffles with the microphone in the far-field. B) Picture of the experiment set up. . . . . . . . . . .

3.1 Comparison of half-power (-3dB) beamwidth (A) and quarter-power ($6 \mathrm{~dB}$ ) beamwidth (B) for the bat biosonar elements. Theoretical minimum beamwidth values are shown as solid black lines for both levels. $D$ is the bat aperture diameter. . . . . . . . . . . . . . . .

3.2 Impact of sidelobes on the beamwidth of the biosonar beampattern samples for noseleaves (gray circles) and pinnae (black x's). Points on the diagonal line do not have sidelobes amplitudes that are large enough to effect the beamwidth estimates. . . . . . . . . . . . . . . . . . .

3.3 Half-power beamwidth values with respect to the aperture diameter $(D)$ to wavelength $(\lambda)$ ratio. A) The HP beamwidths calculated using the first point where the beampattern amplitudes crossed the HP threshold and B) the HP beamwidths calculated using the furthest point where the beampattern crossed the HP threshold. . . . . . . . . . . . . . . . . . . . . .

3.4 Quarter-power beamwidth values with respect to the aperture diameter $(D)$ to wavelength $(\lambda)$ ratio. A) The QP beamwidths calculated using the first point where the beampattern amplitudes crossed the QP threshold and B) the QP beamwidths calculated using the furthest point where the beampattern crossed the QP threshold. 
3.5 Comparison of biosonar and engineered sonar half-power beamwidths to the elliptical-transducer limit values (solid line) as a function of the ratio characteristic dimension $(D)$ over wavelength $(\lambda)$. Biosonar samples are represented by light gray circles and engineered sonars by black triangles. $D$ is a characteristic dimension of the sonar, i.e., array-length for technical sonar and noseleaf or pinna diameter for bat biosonar. . . . . . . . . . .

3.6 Results of the analysis of variance test comparing the scatter of the engineered sonars and biosonars to the reference limit at half-power. For each set of data, the red line inside of the blue box indicates the mean of that set, with the upper and lower edges of the blue box indicating the first and third quantiles. The red crosses represent data points considered outliers within the set. . . . . . . . . . . . . . . . . . . .

3.7 Comparison of the beamwidths of the biological samples with randomly shaped aluminum baffles as a function of the ratio aperture diameter over wavelength $\left(\frac{D}{\lambda}\right)$. Biological samples are shown as gray circles, aluminum baffles as black triangles. . . . . . . . . . . . . . . . . .

3.8 Plot results of the analysis of variance test comparing the scatter of the noseleaf simulates and biosonars to the reference limit at quarter-power. For each set of data, the red line inside of the blue box indicates the mean of that set, with the upper and lower edges of the blue box indicating the first and third quantiles. The red crosses represent data points considered outliers within the set. . . . . . . . . . . . .

3.9 Comparison of beamwidths in two bat families with heavy reliance on active biosonar (horseshoe bats, Rhinolophidae, and Old World roundleaf bats, Hipposideridae, A) and a bat family where the use of active biosonar only occurs as an exception in a few species (Old World fruit bats, Pteropodidae, B). For both families, the QP beamwidth associated with the samples as a function of the ratio of aperture diameter $(D)$ to wavelength $(\lambda)$ are shown along with the elliptical-transducer limit. . . . . . . . . . .

3.10 Results of the analysis of variance test comparing the scatter of the echolocating families (Rhinolophidae and Hipposideridae) and non-echolocating family (Pteropodidae) to the reference limit at quarter-power. For each set of data, the red line inside of the blue box indicates the mean of that set, with the upper and lower edges of the blue box indicating the first and third quantiles. The red crosses represent data points considered outliers within the set. . . . . . . . . . . . . . . . . . . . . 
3.11 Comparison of the distributions for noseleaf (light gray) and pinna samples (dark gray) with respect to the ratio aperture diameter $(D)$ over wavelength $(\lambda) \ldots \ldots \ldots \ldots \ldots \ldots$

3.12 Distance between the beamwidth values of the biosonar samples and the respective lower beamwidth limit as a function of the ratio of characteristic dimension $(D)$ over wavelength $(\lambda)$. A) noseleaf samples, B) pinna samples. $D$ is a characteristic dimension, i.e., aperture diameter for the bat biosonar samples. . . . . . . . . . . . . . . .

3.13 Results of the analysis of variance test comparing the scatter of the reception elements (pinnae) and emission elements (noseleaves) to the reference limit at quarter-power. The comparison was performed only for values of $D / \lambda$ less than 4 to keep the comparison in the regime where noseleaves were typically located. For each set of data, the red line inside of the blue box indicates the mean of that set, with the upper and lower edges of the blue box indicating the first and third quantiles. The red crosses represent data points considered outliers within the set. . . . . . . . . . 36

3.14 Comparison of minimum and maximum beamwidths: A) Minimum beamwidth values matched with the maximum aperture diameters. B) Maximum beamwidth values matched with the minimum aperture diameters. . . . . 38

3.15 The reference beamwidth values is plotted as the black line with respect to aperture diameter $(D)$ to wavelength $(\lambda)$. The scattered points are on a grey scale, where the darker a point is, the flatter the aperture is. . . . . .

3.16 The reference beamwidth value is plotted as the black line with respect to aperture diameter $(D)$ to wavelength $(\lambda)$. Eccentricity is measured by the greyscale of the points on the graph, where the darker a point is, the lower it's eccentricity, i.e. the less circular the aperture is. . . . . . . . . . .

3.17 The eccentricity of the geometry is compared to the eccentricity of the beampattern. The black line in the middle represents the expected behaviour for elliptical-transducers. The grey o's represent noseleaves while the black x's are pinnae. Each of the points on the graphs is the average eccentricity of the beamwidths for the aperture over its 10 measured frequencies. . . . . . . . . . . . . . . . . 
3.18 Distance between the beamwidths of the biosonar samples and the respective elliptical-transducer limit as a function of the diameter to wavelength ratio $\left(\frac{D}{\lambda}\right)$. The beamwidth data has been arranged in bins of width $0.25 \frac{D}{\lambda}$. The averages for all data points in each bin are indicated by open circles; the error bars mark the standard deviations. . . . . . . . . . . . . . 


\section{List of Tables}

3.1 Results of the analysis of variance test comparing the scatter from the limit of the biosonar apertures and the engineered sonars. All comparisons were performed on the half-power beamwidth data. SS is the sum of squares, $\mathrm{df}$ is the degrees of freedom, MS is the mean squared error and $\mathrm{F}$ is the $\mathrm{f}$ statistic, which is the ratio of the mean squared error of the groups to the mean squared error of the error. . . . . . . . . . . . . . . . .

3.2 Results of the analysis of variance test comparing the scatter from the limit of the biosonar apertures and the noseleaf simulates. All comparisons were performed on the quarter-power beamwidth data. SS is the sum of squares, $\mathrm{df}$ is the degrees of freedom, MS is the mean squared error and $\mathrm{F}$ is the $\mathrm{f}$ statistic, which is the ratio of the mean squared error of the groups to the mean squared error of the error. . . . . . . . . . . . . . . . .

3.3 Results of the analysis of variance test comparing the scatter from the limit of the echolocating families (Rhinolophidae and Hipposideridae) and the non-echolocating family (Pteropodidae). All comparisons were performed on the quarter-power beamwidth data. SS is the sum of squares, df is the degrees of freedom, MS is the mean squared error and F is the f statistic, which is the ratio of the mean squared error of the groups to the mean squared error of the error. . . . . . . . . . . . . . . 
3.4 Results of the analysis of variance test comparing the scatter of the reception elements (pinnae) and emission elements (noseleaves). All comparisons were performed on the quarter-power beamwidth data. The comparison was performed only for values of $D / \lambda$ less than 4 to keep the comparison in the regime where noseleaves were typically located. SS is the sum of squares, $\mathrm{df}$ is the degrees of freedom, MS is the mean squared error and $\mathrm{F}$ is the $\mathrm{f}$ statistic, which is the ratio of the mean squared error of the groups to the mean squared error of the error. . . . . . . . . . . . .

5.1 Table Containing Geometry and Beamwidth Data for Samples sorted by Scientific Name. Species denoted as sp. represent an unknown species within a genera. Beamwidths given as the half-power beamwidths for that aperture at a given frequency excluding sidelobes. The 10 frequencies are equally spaced between the minimum and maximum frequency. . . . . .

5.2 Table Containing Geometry and Beamwidth Data for Samples sorted by Scientific Name. Species denoted as sp. represent an unknown species within a genera. Beamwidths given as the quarter-power beamwidths for that aperture at a given frequency excluding sidelobes. The 10 frequencies are equally spaced between the minimum and maximum frequency. . . . 


\section{Chapter 1}

\section{Introduction}

\subsection{Background}

Engineered sonar is of pivotal importance for sensing in underwater environments, where sound waves are able to propagate over long distances at high speeds, whereas electromagnetic waves such as visible light are severely limited in range [49].

In-air sonar follows the same basic principles as underwater sonar, but has to deal with higher absorption and lower sound speed which result in less favorable conditions [49]. Nevertheless, the capabilities of echolocating bat species show that sonar can support the sensory information needs of highly mobile mammals [29] - although at ranges that are short compared to the operational ranges of underwater sonar.

A system characteristics frequently used to describe biosonar and technical sonar alike is the beampattern $[2,29]$, i.e., the distribution of emitted signal energy or the receiver sensitivity over direction angle and frequency [49].

In engineered sonar, the primary criterion for judging the utility of a given beampattern is the concentration of pulse energy or receiver sensitivity over direction angle. The narrower the beam, i.e., the smaller the beamwidth, the higher the sonar's resolution [49]. The ability of any given sonar emitter or receiver to produce a narrow beam is limited by how large or small its aperture is compared to the employed wavelengths. A lower limit on the beamwidth can be derived from the ratio of wavelength to diameter of the sonar aperture [2]; the larger the aperture of a sonar is compared to the wavelengths it operates on, the narrower a beam it is capable of creating. 

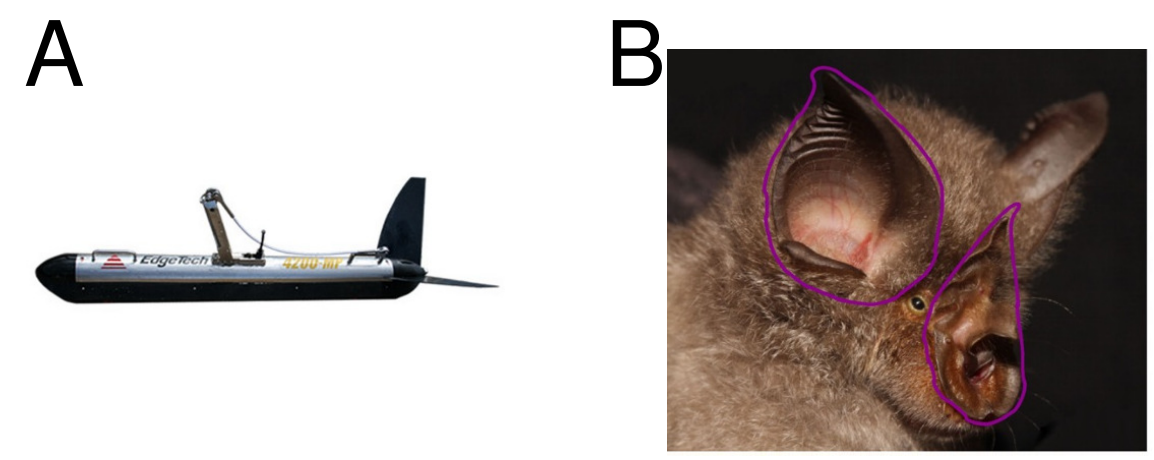

Figure 1.1: A look at an engineered sonar system vs biosonar elements. A) An Edgetech 4200 Side Scan sonar system that contains sensors along it's $125 \mathrm{~cm}$ length [5] (Picture Copyright Edgetech, reproduced with permission) and B) A bat from the Rhinolophidae family with its right pinnae (outer ear) and noseleaf circled in purple.

In order to create large apertures and the corresponding narrow beams, engineered sonars tend to consist of large numbers of emitting and receiving elements working together in an array. The incoming and outgoing signals are combined across all elements in the array to produce a narrow sonar beam that is used to scan the environment [2]. The downsides of this approach are the large size of the array and the large number of elements that are needed to cover it, as well was the computationally expensive operations that are needed to combine the signals from a large number of elements.

In contrast to engineered sonar, the biosonar of bats operates with only three elements [29]: one emitter, the nose or the mouth (depending on species), and two receivers, the outer ears (pinnae). Bat biosonar operates in air and the overall system size is much smaller compared to the wavelengths used than is typically the case for engineered sonar (s.Figure 1.1). The technical sonars presented in this work range from operating frequencies of $42 \mathrm{kHz}$ [21] to $1.8 \mathrm{MHz}$ [23], though a majority of the sonars discussed have operating frequencies between $200 \mathrm{kHz}$ and $900 \mathrm{kHz}$ [33, 17, 47, 45, 46, 40, 51, 5]. Bat biosonar has a frequency range between $11 \mathrm{kHz}$ and $212 \mathrm{kHz}$ [13] but most bats call with frequencies between $20 \mathrm{kHz}$ and $60 \mathrm{kHz}$ [13]. Due to their substantially lower frequencies combined with much smaller sizes, bats are in a much less favorable conditions than engineered sonars when it comes to forming narrow beams.

The goal of the work presented here has been to compare beamwidth in biosonar and engineered sonar with each other and to the theoretical beamwidth limit for the respective ratio of sonar aperture size and wavelength. Closeness to the theoretical limit could be 
seen as an indication that a small beamwidth is an important parameter for the function of the respective system. In addition, an experimental data set with noseleaf simulate baffle shapes with random internal geometry and imperfections has been acquired to serve as a reference for how close random shapes of a given size will cluster around the limit threshold. All these comparisons should shed light on the question whether beamwidth has been as critical to the evolution of bat biosonar as it is has been to the design of engineered sonar.

\subsection{Review of Literature}

\subsubsection{Bioinspiration and Biomimetics}

Bioinspiration is the study of materials, designs, structures and behaviors of biological specimens with the goal of adapting these ideas into technical and synthetic designs. Similarly, biomimetics, or biomimicry, is the study of imitating, or mimicking, natural designs to solve complex problems [50]. Both of these ideas form from the same base concept: taking advantage of nature's billions of years of evolution to gain insight into how natural organisms or systems have solved complex or difficult problems. While a search of the literature reveals the keywords of bioinspiration and biomimetics didn't start regularly appearing in literature until the turn of the century, the concept of using nature as guidance for design was not a new idea. A very widely used and well known example of this, Velcro, first came about due to the inventor noticing burdocks (seeds from the genus Arctium), sticking to his clothes [12].

Many contemporary researchers however actively look into natural and biological systems for inspiration. One of the largest fields currently benefiting from bioinspiration is the field of robotics [36]. Bioinspiration has lead to new developments and ideas such as soft robotics which uses highly flexible materials, instead of rigid materials, to allow a larger and more natural set of motion [16] or the creation of new artificial polymers from studying artificial muscle [31]. Bioinspiration has been applied to more than just mechanical problems. It has been used heavily in chemistry as a basis for creating composite materials [7] or materials with unusual properties such as superwettability [43]. It has also been used to design new types of sensors and sensing paradigms, such as spider-inspired

flexible hair sensors [22] or, of important relevance to this work, bat biosonar inspired sensing platforms [4].

Bioinspiration and biomimetics have been trending in engineering research. For the past 
twenty years, the number of conference papers, journal papers, and patents using bioinspired or biomimetic technology has continually increased each year as the importance and relevance of looking at nature for inspiration in engineering designs has continually proven its worth. Bioinspiration and biomimetics have been applied to an incredibly large and varied array of different engineering problems as engineers and scientists continue to use nature's billions of years of evolution as a springboard for new ideas and concepts.

\subsubsection{Bats and Biosonar}

Bats have proven to be a highly diverse and successful group of mammals, accounting for over $20 \%$ of all mammal species [29]. While they are not the only species to use echolocation to navigate, bats are the most researched species in terms of echolocation and biosonar. There has been significant research conducted into the echolocation behaviors of bats, particularly the hunting of insectile prey, stretching back to the 1960s [10] and continuing throughout the twentieth century and into the twenty-first century with other researchers performing similar investigations and analysis of echolocation behaviors except for differing families or genera of bat [38, 15, 37]. Much of this early work in biosonar $[10,38,15,37]$ has had an emphasis on collection of field data in order to describe how the differing families of bat operate within their environments, focusing on their operating conditions, such as flight speed, distance needed to acquire targets, etc, and the capabilities of the bats themselves rather than having a focus on what could be learned and applied from how the biosonar performs.

In addition to the study of the natural environments, there was initial research done in the early 1990s into using bat-like sonars on robots [19]. These robots were setup with a sonar system that mimicked the sensor configuration of bats [1]. The bat-like system was used in experimentation based on the context of prey capture [19] as well as obstacle localization [1] and the results of the experiments were that the bat-like sonar represented an efficient sensing system.

An article published in 2007 [28] is one of the first sources discussing the differences between biosonar and technical sonars as well as the merits of incorporating biosonarinspired approaches. This article brought up many important challenges facing the design of biosonar, such as dynamics in the sensors during emission and reception, challenges in transducer performance, the actuation and integration of beamforming baffle shapes, and the miniaturization of the sonar system among others [28].

Since the publication of the 2007 article, there has been a significant amount of research invested into examining what can be learned from biosonar and applied to technical sonar. 
One of the differences between technical and biosonar that has been observed and studied in literature since has been the dynamics of the biosonar system [3, 26, 25, 9]. This has been of particular interest due to the novelty of the idea: bending the biosonar apertures to dynamically alter a sonar signal [3]. The apertures have been found to change their shape by up to $20 \%$ of their total length within 100 miliseconds while emitting or receiving sound [25]. The effect of the dynamics on the emission signal has been studied and found to produce strong time-dependent characteristics in elliptical sound outlets similar in size and shape to those used by horseshoe bats [9].

In addition to information on changes in the beampattern due to dynamics of the system, recent research has begun to show that these changes in beampattern can dynamically encode target information into the signal [44]. This branch of research has changed from simply showing differences to showing that the differences in signal is able to encode additional information, an exceedingly useful tool for technical sonars that is not used or well understood and hasn't been extensively studied. Sonar systems designed to use these dynamics and outfitted with biomimetic apertures have been developed and show the potential of the bioinspired system as a new paradigm in sonar sensing, though significant research is still required to be able to make full use of the biosonars unique characteristics [4].

Other research has been conducted into recreating biosonar beampatterns from the geometry of the biosonar apertures [54]. Combined with analysis of the static biosonar beamforming mechanism and strategies [24], there is now the ability to create acurate, numerically derived beampatterns and beam information for biosonar apertures that can be used to gain new insights into how to incorporate bioinspired strategies to help solve problems facing sonar today.

\subsubsection{Review of Technical Sonar Literature}

Research in sonar and the propagation of sound underwater is an old field of study, dating as far back in literature as the discovery and documentation of primitive passive sonars by Leonardo da Vinci in 1490, but began to resemble its modern form of research starting in World War II, where quantitative and precise measurements of undersea environments became more and more important [49]. Since World War II there has been a large amount

of research on sonar and sonar capabilities due to the important nature of the marine environment in warfare and navigation. 


\subsubsection{Sonar Concepts}

As previously mentioned, the basis of sonar is the study of the propagation of acoustic waves in a medium, typically water [2]. Sonars can be split broadly into two categories, passive and active, where passive sonars only listen to incoming acoustic waves while active sonars emit their own acoustic waves and listen for the echoes of their signals to return [2]. This work and most of the following section will focus on discussions as they relate to active sonars, but that isn't to dismiss the importance of passive sonar or to imply that the same physical principles and research does not also apply to passive sonar.

With an important goal of active sonar being to survey the surroundings of the craft, whether looking at the local topography or attempting to find and track other objects, such as undersea mines or submersibles [8], there has been a large amount of focus placed on controlling the beams of the sonar systems. Beamforming is a signal processing technique that is utilized to increase the directivity of sonar arrays by controlling the phase and amplitudes of the many transmitters in an array in order to create constructive and destructive interference in the acoustic wave [48]. As beamwidths, a measure of sonar resolution, are dependent on the size and frequencies at which a transmitter is operated at [49] and there are physical constraints imposed in the creation and use of transmitters, beamforming has an important role in the development of very high resolution sonars.

Beamwidths are primarily measured in the main lobe of a sonar signal, which is the lobe of the sonar signal that contains the maximum gain, and is typically sent in the direction that the sonar is attempting to look at. However, many sonars have naturally occurring, or induced through beamforming, sidelobes in the signal that are lobes of the sonar signal with local maxima that are not the main lobe. Sidelobes, especially larger sidelobes, have potential to interfere with the sonar signal and research has been conducted on how to reduce them in arrays, such as by using older methods like nonuniform element spacing in the array [11] or newer methods like flexible genetic algorithms for pattern synthesis [52]. Whichever method is used, the end goal is to reduce the magnitude of the sidelobes so that they do not interfere with the signal detection of the main lobe. While still an important subject in undersea sonar, much of the contemporary research for sibelobe reduction is in the area of antenna arrays and wireless communication.

\subsubsection{Trends in Sonar Research}

In recent years, research in sonar arrays has become very wide and varied to match the many innovations in the field. There has been an increasing amount of research into 
miniaturization of submersible systems and the addition of sonar to unmanned underwater vehicles (UUVs). Even narrowing down research to these areas results in research with drastically different aims, such as creating UUVs with small, economically efficient sonars [14], or creating miniaturized UUV's able to operate in alien or non-terrestrial environments like the beneath the frozen crust of Europa [30]. In these situations the large arrays in use by contemporary sonar engineers would be too expensive or too large for the mission, so research continues into finding ways of making the sonar systems smaller while still retaining the resolution and capabilities of larger sonar.

Much of the current research increasing the capabilities of sonar is being focused on the processing of sonar signals rather than development of new hardware. Researchers are attempting to use methods such as matched filtering theory [53] or Bayesian localization [32] with simple sensors or sensor arrays in order to increase the performance of the sonar that is typically reliant on the size and number of sensors, not processing. A quick search of literature reveals that in the previous year, 2016, there were more than twice as many articles published relating to sonar software than sonar hardware.

These two trends in publications and research in sonar, miniaturization for UUV and increased sensing and processing capabilities, also point towards another emerging trend in sonar research: the use of autonomous UUVs to perform dangerous or tedious tasks. Of particular interest in the rising study of autonomous sonar controlled systems are those used for clearing undersea mine fields [42] on the military side of research and using autonomy in UUVs for marine archaelogy [27] and surveillance of natural resources and sites, such as coral reefs [20].

\subsubsection{Conclusion}

Throughout much of the contemporary research the bat biosonar system has been approximated or represented as an elliptical transducer or a baffled elliptical transducer. There hasn't been any direct comparisons between the expected performance of appropriately sized transducers or sonar arrays and the biosonar arrays while several publications have sought to look into factors, such as dynamics, that could contribute to shortening a perceived performance gap between biosonar and technical sonar. Meanwhile, engineered sonar is constantly seeking new methods of improving performance without increasing the size or cost of the sonar. In the following work, biosonar apertures will be compared to engineered sonars, showing the performance gap in terms of sensing resolution between the biosonar systems and contemporary engineered sonars. 


\subsection{Problem Statement and Research Objectives}

\subsubsection{The Case for Studying Biosonar}

There are many works on biosonar that attempt to use measurements of bats in their natural habitat to make statements about broad capabilities of biosonar [38, 15, 37]. It is difficult to obtain precise numerical beampatterns directly from bats which would require having a bat naturally emitting its sonar into a large microphone array. Difficulties with creating experimental beampatterns directly from bats led to development of numerical methods that can be used to simulate the beampatterns of biosonar apertures [54].

Much of the contemporary research on biosonar is focused on operational differences between biosonar and technical sonar, such as the dynamic motion of the biosonar aperture while emitting or receiving sound [25]. Part of the reason for this research is that there are perceived, or known but not quantified, differences between biosonar and technical sonar. A quick inspection of sizes shows that there is indeed a significant size difference between biosonar apertures and technical sonar arrays, lending merit to the concept that biosonar and technical sonar operate differently. However, to this point prior research has not quantified this difference to see if the perceived difference is as large as initial inspection would make it out to be. Additionally, it is known that engineered sonars attempt to minimize their beamwidths as much as physically possible, but it is unknown whether bats attempt to have the same minimization.

By creating a method for comparing biosonar systems to equivocal engineered sonar systems or reference baffles, the amount of difference between biological and technical sonar can be quantified. Additionally, creation of a large data set of beampatterns and examination in terms of their beamwidths allows examination in new areas to investigate biosonar and better understand how it works.

\subsubsection{Research Objectives and Hypotheses}

Prior to the research presented here, it was qualitatively obvious that bats operated in much smaller regimes of diameter over wavelength. This operational difference can be seen in the size of the biosonar apertures (5mm to $20 \mathrm{~mm}$ typical, s. Table 5.1 ) compared to engineered sonars $(300 \mathrm{~mm}$ to $1.25 \mathrm{~m}$ typical for sonars used in this research $[33,17,34,18,51,47,41,45,46,35,40])$ and the operating frequencies $(20 \mathrm{kHz}$ and $60 \mathrm{kHz}$ typical for bats [13] vs $200 \mathrm{kHz}$ to $900 \mathrm{kHz}$ typical for the sonar investigated in this 

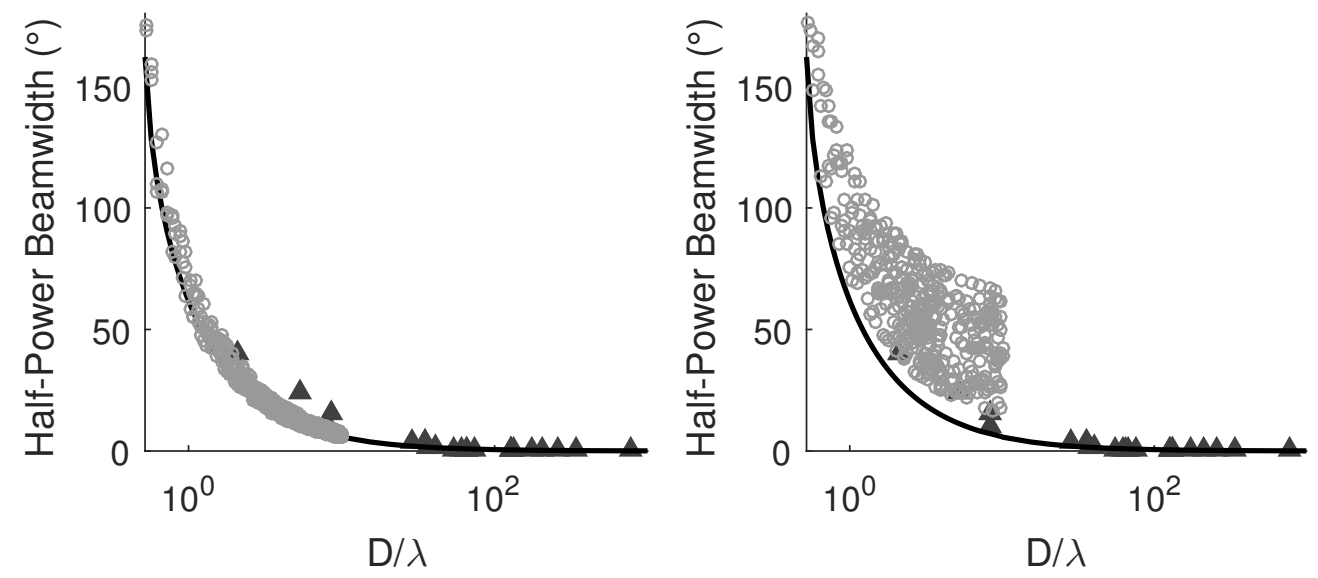

Figure 1.2: Visual representation of the first hypothesis tested in the current work: A) shows the first form of the hypothesis, that the engineered sonars, represented by black triangles, and biosonars, grey dots, have similar scatter and closeness to the reference limit represented by the black line. B) shows the alternative hypothesis that the biosonar has a larger scatter.

research $[33,17,47,45,46,40,51,5])$. As beamwidth can be measured as a function of diameter over wavelength, with larger values of diameter over wavelength leading to narrower beamwidths [2], it can be surmised that the biosonar beamwidths will be larger than the engineered beamwidths. Nevertheless, the following four main research questions remain open when comparing bat biosonar and engineered sonar:

Engineered sonars follow reference equations for beamwidth as a function of diameter over wavelength and have very little scatter from these limits. The first hypothesis that was tested is that biosonar will have similar scatter from the limit as engineered sonar, with the alternative hypothesis being that the biosonar will have more scatter (s. Figure 1.2).

The second hypothesis that was tested is that aluminum noseleaf simulates with random imperfections and internal geometry will have the same scatter as the biosonar apertures do, with the alternative hypothesis being that either the biosonar or noseleaf simulates will have less scatter (s. Figure 1.3).

The third hypothesis that was tested is that families of bats that use echolocation have biosonar apertures that operate in the same regimes of diameter over wavelength and beamwidth as families of bat that do not use echolocation, with the alternative hypoth- 

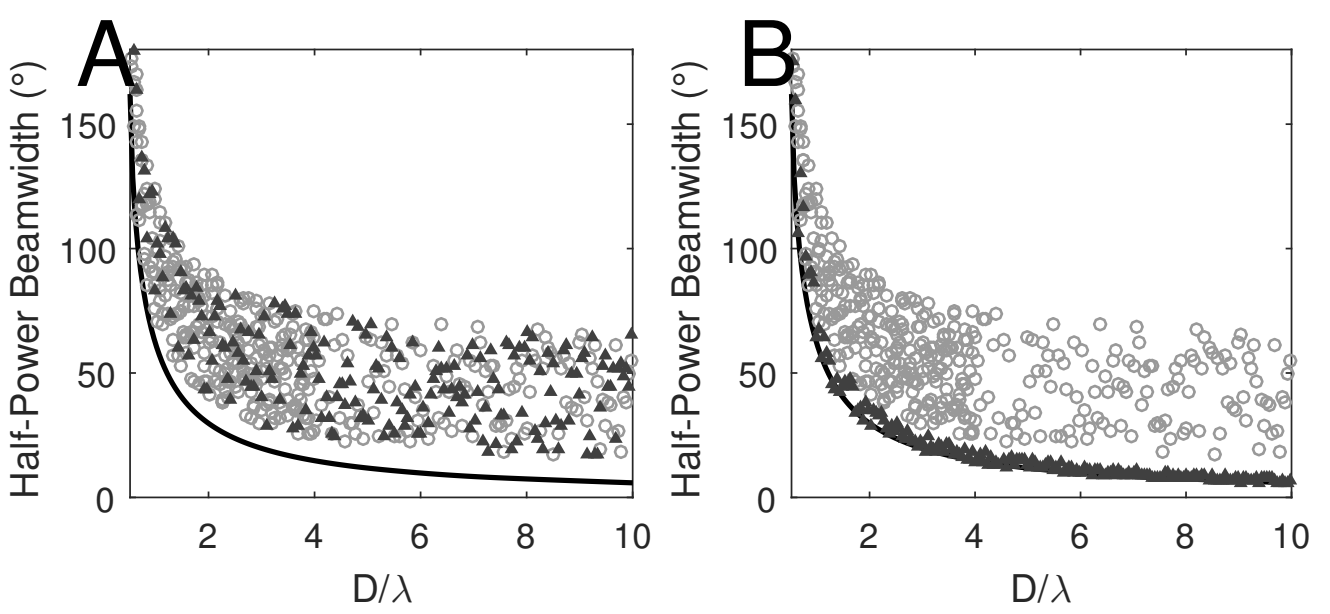

Figure 1.3: Visual representation of the second hypothesis to be tested in the current work: A) shows the first form of the hypothesis, that the aluminum baffles, represented by black triangles, and biosonars, grey dots, have similar scatter and closeness to the reference limit represented by the black line. B) shows the alternative hypothesis that either the reference baffles or biosonar has less scatter.

esis being that families that use echolocation and families that do not use echolocation operate in different regimes of diameter over wavelength or beamwidth.

The final hypothesis that was tested is that noseleaf (emission) elements operate in the same regimes and with the same beamwidths and scatter as pinnae (reception) elements, with the alternative hypothesis being that they operate in different regimes.

In order to test these hypotheses, several sets of research objectives were created. The first was the creation of a framework within which the biosonar apertures could be compared to engineering sonars. This objective included selection of suitable engineering analogues for the biosonar apertures as well as the creation of a methodology for converting the biosonar into the chosen analogue. The second objective of the research was to take numerically generated biosonar beampatterns and find the corresponding beamwidths. The final objectives of the research were to use the large database created to test the hypotheses and look for additional insights or areas for future research. 


\section{Chapter 2}

\section{Methods}

\subsection{Biosonar Apertures and Conversion to Engineering Analogues}

\subsubsection{Biosonar Aperture Data}

The biosonar apertures used in this work are all taken from computer micro-tomographic $(\mu \mathrm{CT})$ scans of bat noseleaves and pinnae. The bats scanned were dead and had been preserved in a low-concentration ethanol solution prior to scanning. There were over 500 different $\mu \mathrm{CT}$ scans of bat apertures from over 300 different bats. After scanning a specimen the relevant apertures were cut out from the rest of the mesh so they could be examined by themselves.

After all of the apertures were scanned, it was necessary to check meshes manually in order to verify that there were no problems with the scan. While there were some $\mu \mathrm{CT}$ meshes that had easily identifiable problems, such as artifacts or other problems with the scan, a more time consuming aspect of filtering was making sure that the examined samples themselves appeared to be in good condition. In this context, good condition meant that the aperture was completely intact and in an upright neutral position. Pictures and videos of families of bats were used to identify whether pinnae or noseleafs were bent, damaged, or had other problems that would make them poor choices for comparison.

Additionally, all of the samples that were found by manual inspection to be in good condition had beampatterns generated using methods described later in Chapter 5. Some 
meshes had problems not apparent to visual inspection that caused the numerically generated beampatterns to fail to converge, resulting in meshes that geometry could be obtained from but not beampattern data. Since both geometry and beampattern data would be required for full comparisons of the biosonar apertures, meshes that failed to produce numerical beampatterns were also discarded.

The final set of apertures were comprised of a total of 151 shape samples taken from 137 different bats. There was only one sample where both pinnae and the noseleaf was well preserved enough to use all three of its apertures for comparison, but there were 12 different bats were both ears were preserved well enough to be used. Of the 151 aperture samples there were 61 noseleaves and 90 pinnae. While it might be expected that there would be twice as many pinnae compared to noseleaves, since bats have two pinnae and only one noseleaf and there were more pinnae in the scans, the noseleaves appeared to withstand preservation and scanning better than the pinnae resulting in a higher percentage of them being usable for comparison.

The pinnae and noseleaves came from 8 different bat families, with the most prominent family being the horseshoe bats (family Rhinolophidae) from which 21 noseleaf samples and 24 pinna samples were analyzed. The other bat families represented in the data set were: Hipposideros (23 samples), Emballonuridae (5 samples), Molossidae (2 samples), Megadermatidae (1 sample), Nycteridae (15 samples), Phyllostomidae (20 samples), Pteropodidae (4 samples), and Vespertilionidae (36 samples).

\subsubsection{Conversion from $\mu$ CT Scans to Engineering Analogue}

In order to approximate the 3D mesh of the biological structures by a $2 \mathrm{D}$ transducer the point cloud mesh is taken through a series of simplifications and processing. The first step is edge filtering the mesh, finding edges whose dihedral angle is greater than $15^{\circ}$. The result of this is that only heavily curved edges, such as those along the outer edge of the aperture, are kept, discarding much of the internal geometry. A point along the outer edge of the aperture was manually selected and the rest of the points along that edge are selected by using a point clustering, nearest neighbor algorithm. The majority of the apertures were able to use a hop-through distance in the point clustering algorithm of $0.1 \mathrm{~mm}$, but 17 of the 151 samples required additional manual cleaning or different filtering in order to select just the outer edges of the aperture. Once the outer edges of the structure were determined, a plane of best fit was found using least squared of normal distance. Finally, the points from the edge were projected onto the plane of best fit and then the minimum and maximum length of the aperture through the centroid were found (s. Figure 2.1). The 

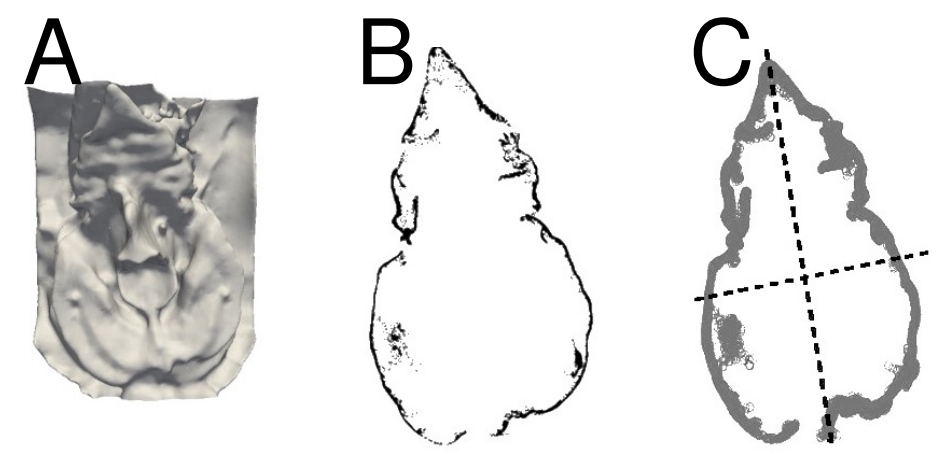

Figure 2.1: Approximation of the biological shape samples by a planar elliptical transducer: A) $\mu$ CT model of a noseleaf from a Sulawesi horseshoe bat (Rhinolophus celebensis), B) Point cloud resulting from detecting edge vertices in the mesh, C) Edge vertices projected into the fitting plane with lines along the minimum and maximum aperture lengths (dashed lines).

code used to convert the $\mu \mathrm{CT}$ scans to the $2 \mathrm{D}$ engineering analogues is given in Appendix A.1: Geometric Processing Code. This code is provided so that other researchers can replicate the results or use the code to generate additional data points for new apertures.

The minimum and maximum diameters were chosen to be found through the centroid for easier comparison with technical sonars and transducers. As will be discussed in more depth later, beamwidth for a sonar is typically measured through maximum gain of the signal, which in transducers and other planar arrays aligns with the centroid of the transducer or array. Consideration was given on whether to use the measured maximum and minimum or to use the length perpendicular to the maximum as the minimum, as would be the case of a perfectly elliptical transducer, but the decision was made to calculate maximum and minimum separately due to the non-uniformity of the shapes and beampatterns. This choice in calculating minimum and maxmimum seperately had a larger effect on noseleaves than pinnae, as the pinnae have relatively uniform and consistent shape while some noseleafs, such as those of the trident bats (Aselliscus stoliczkanus), have peculiar and nonuniform shapes. 


\subsection{Beamwidth Determination for Biosonar Apertures}

\subsubsection{Importance of Beamwidth}

Beamwidth is a widely used metric by sonar engineers for evaluating the performance of sonars. Beamwidths represent the resolution with which a sonar can sense its environment and is measured in degrees. Of the two categories of sonar, active and passive, this work is concerned primarily with active sonar, that is sonars that emit sound pulses and listen for the echoes of those pulses [2]. Having a narrower beamwidth represents the ability to more accurately and precisely direct energy, which in addition to the benefit of increasing your sensing resolution can also help reduce interference between multiple sonar systems and help reduce the signature of the sonar carrier. These advantages to having narrow beamwidth has lead to beamwidth being a heavily discussed and documented metric for engineered sonars which makes it a valuable metric for being able to make quick performance comparisons to a wide array of sonar systems.

\subsubsection{Planar Array Reference Beamwidth}

For comparison with this reference, the noseleaves and pinnae were treated as unbaffled 2D elliptical transducers. To compute the ratio of diameter $(D)$ over wavelength $(\lambda)$, the maximum diameter of the biosonar element was matched with the minimum beamwidth and the minimum diameter of the biosonar with the maximum beamwidth.

A reference lower beamwidth limit used for comparison to the data was derived from the equation for a continuous planar circular array in an infinite baffle (s. Equation 1, [49]). This equation also covers infinitely baffled circular planar arrays of diameter $D$ or a single element of diameter $D$ and a wavelength $\lambda$ :

$$
b(\theta)=\left[\frac{2 J_{1}[(\pi D / \lambda) \sin (\theta)]}{(\pi D / \lambda) \sin (\theta)}\right]^{2}
$$

where $b(\theta)$ is the beampattern response at angle $\theta$ and $J_{1}[]$ is the first-order Bessel function.

The choice to use an infinite baffled circular planar array as the reference instead of a specifically baffled array or a conical horn was made to find common ground between biosonar apertures and technical sonar. An infinitely baffled system was chosen so that an arbitrary baffling would not be used. While some biosonar apertures do appear from visual inspection to bear resemblance to baffled transducers, the amount of baffling and 
it's arrangement varies from species to species. The choice to use a planar array rather than a conical horn was made for similar reasons. By using a planar array, the dimensions that need to be controlled for are decreased to just minimum and maximum diameters, so there is no need to choose arbitrary dimensions for length, curvature, etc, that vary heavily from one bat species to another.

\subsubsection{Generation of Numerically Derived Beampatterns}

Predictions of bat biosonar beampatterns were derived numerically from digital models of the geometries of bat noseleaves (emission baffles used by bats with nasal emission of biosonar pulses) and pinnae (outer ears, i.e., the sound receivers in bat biosonar) [3]. The digital models were taken from $\mu \mathrm{CT}$ scans and the geometries were treated as a cubic finite-element mesh with a perfectly reflecting boundary condition to represent the boundary between the biological tissue and air [24]. Using these meshes and boundary conditions, the near field was solved for by using the wave equation which, given the stationary and space-dependent nature of the beampattern, was expressed by the Helmholtz equation [54]. Using the complex field values from the finite-element model, the propagation of the acoustic wave into the far-field was handled using the Kirchoff integral formulation [24].

The ultrasonic beampatterns of each of the 151 pinnae and noseleaves samples were evaluated at 10 frequencies evenly spaced throughout the operating frequency band that has been documented for respective bat species in the literature.

\subsubsection{Biosonar Beamwidth Evaluation}

The minimum and maximum beamwidths of the biosonar samples were determined from the numerical beampattern estimates as follows: First the beampatterns were rotated so that the angle associated with the maximum gain, i.e., the maximum response angle (MRA) was pointed in a known direction. This was done both to make the next steps of the process simpler and so that the beampatterns could all be aligned in the same direction for visual inspection and comparison of size and shape. After this, the beampattern was cut by a series of planes each of which contained the vector from the origin to the MRA. A family of planes was created by rotation around the vector of the MRA. A plane was placed every $0.5^{\circ}$ of rotation angle to sample the entire two-dimensional beampattern (gain as a function of two angles, e.g., azimuth and elevation) with high resolution. Each slice of the beampattern produced a one-dimensional function (normalized beam gain as a function of a single angle). A beamwidth was calculated for each of the slices, looking for 
the minimum and maximum beamwidth across all slices. The maximum and minimum beamwidths are found at two different levels: $-3 \mathrm{~dB}$ (half-power, HP) and -6dB (quarterpower, QP, or half-voltage, s. Figure 2.2).

In addition, some of the beampatterns had prominent sidelobes which contained values higher than HP or QP that are separate from the main lobe of the sonar signal. For the purpose of the comparison in this work, beamwidths have been calculated in two different ways - with and without taking sidelobes into account. Beamwidth estimates not including sidelobes were obtained by traversing the one-dimensional beampattern from the MRA outward until the amplitude dropped below the respective threshold $(-3$ or $-6 \mathrm{~dB})$ for the first time. In order to get beamwidth estimates including sidelobes, each beampattern was traversed starting at angles 180 degree away from the MRA and the beamwidth was determined by the first angles were the beampattern gain exceeded the threshold for the first time.

There were two primary pieces of code used in the beamwidth evaluation. The first piece is given in Appendix A.2: Beampattern Processing Code and takes a beampattern, numerically or experimentally generated, in the form of gain as a function of azmiuth and elevation angles and performs the interpolation to create the 2D slices of the beampattern as well as solving for the HP beamwidth excluding sidelobes. An additional piece of code given in Appendix A.3: Beampattern Post Processing Code takes the 2D beampattern slices saved from the previous code and calculates several additional values, such as QP beamwidth, beamwidths including sidelobes, locations and values of the nulls, etc. These two pieces of code are provided so that readers and future researchers can either replicate the results seen in this research or use the code to generate additional data for beampatterns not used in this research.

\subsubsection{Half-Power vs Quarter-Power Beamwidths}

In engineered sonar, it is standard to use the half-power beamwidth [2], however some of the comparisons for the biosonar in this paper were done using the quarter-power beamwidth. The reason for this was that some of the sampled biosonars have sidelobes near the MRA that were included in the beamwidth at the - $6 \mathrm{~dB}$ level, but not for the $3 \mathrm{~dB}$ level. Additionally, some biosonars have ripple in the signal thresholds where there is noise in the signal that is too small to be accurately described as a sidelobe but can have small impacts on the measurement values. The $-6 \mathrm{~dB}$ threshold is used for many comparisons between biosonars due to the beamwidths being larger and having had more

time for ripple and noise in the signal around the MRA to have dispersed, resulting in a 

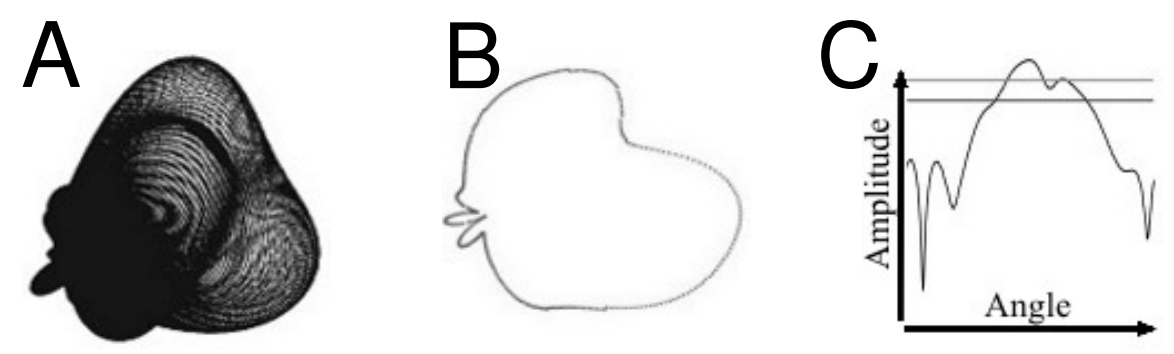

Figure 2.2: Conversion of two-dimensional, numerical beampattern estimates into onedimensional beampattern functions: A) a numerical beampattern estimate derived from the Sulawesi horseshoe bat (Rhinolophus celebensis) shown in Figure 2.1. B) One of the beampattern slices obtained at different rotation angles, shown in polar coordinates. C) The same beampattern slice shown in B) converted in to Cartesian coordinates. The horizontal lines indicate the $-3 \mathrm{~dB}$ and $-6 \mathrm{~dB}$ threshold levels

smaller impact on the beamwidth. Comparisons with technical sonar were performed using the half-power value as many manufacturers do not have public information on their quarter-power beamwidth.

\subsection{Acquisition of Technical Sonar Data}

\subsubsection{Military Sonar vs Commercial Sonar}

Engineered sonars additionally can be split into two categories: commercial and military. This work will use metrics solely from commercial sonars for comparison to biosonar due to the difficulty in obtaining publishable specifications for military sonars. However, it should be noted that military sonars tend to be of larger size and higher quality than their commercial counterparts. Of the commercial sonars used in this thesis, the largest sonar is the Sonardyne Solstice SSS, which is $1.25 \mathrm{~m}$ long [5]. For comparison, the bow array of a Seawolf SSN-21 submarine has a 24 foot (7.3 meter) diameter making it more than five times as large. Due to the physics governing size and beamwidth, it is easy to understand even without seeing any technical specifications and capabilities that these massive sonar arrays would likely be an order of magnitude higher in terms of their resolution from the 
discussed commercial sonars. Additionally, the technical sonars discussed in this paper are contemporary sonar, commercially available at the time of the comparison.

\subsubsection{Technical Sonars Used for Comparison}

The data set of engineered sonar was compiled from 23 different technical sonar devices. The beamwidths and sizes for the engineered sonar were taken directly from manufacturers' specifications $[33,21,17,6,34,18,51,47,41,45,46,35,40]$. These sonars had operating frequencies ranging from $42 \mathrm{kHz}$ [21] to $1.8 \mathrm{MHz}$ [23]. Several of the sonars were dual-beam or multi-beam, meaning that they had two or more operating frequencies which would produce two different sized beamwidths [33,35,34]. Most commonly for these sonars the higher frequency would be double the lower, which resulted in the higher frequency having half the beamwidth of the lower frequency [33, 34], which highlights the relative linearity of decreases in beamwidth with respect to array-length to wavelength at high values of array-length to wavelength. The smallest of the sonars had a diameter of $16.4 \mathrm{~mm}$ [21], which is comparable to some of the larger biosonar apertures, and with it's low frequency placed it at scale of diameter to wavelength ratio equivocal to the biosonar apertures. The largest of the engineered sonars was $1.25 \mathrm{~m}$ long [5] which, combined with its high operating frequency, resulted in a diameter to wavelength two magnitudes larger than the average biosonar.

\subsection{Aluminum Random Emission Baffle Experiment}

Having compared the biosonar apertures to technical sonar and found differences in performance, both in how close to the limit the apertures were and the area of operation, an experiment was designed to test and see if the biosonar apertures performed better than randomly produced aluminum baffles, which would be operated in the same aperture diameter $(D)$ to wavelength $(\lambda)$ ratio as the biosonar apertures. If the biosonar was found to operate similarly, or worse than, randomly generated baffles it would serve as additional indication that beamwdith may not have been an important evolutionary consideration for biosonar. Additionally, the aluminum baffles provide a reference point for how randomly generated baffles of the approximate size of bats compare to the reference equations for an unbaffled planar array used as the reference limit. The baffles being close to the limit would indicate that the unbaffled planar array serves as an acceptable approximation of short, conical, baffled transducers. 


\subsubsection{Equipment and Setup}

The chirp for the experiment was produced by two Senscomp 600 loudspeakers. The loudspeakers had a $100 \mathrm{~mm}$ long waveguide attached to them that went from the two $42 \mathrm{~mm}$ loudspeaker diameters to two separate $2 \mathrm{~mm}$ diameter exits. The noseleaf simulate baffles were attached to the ends of the waveguide. The acoustic properties of the noseleaf simulates were tested using a linear-frequency modulated chirp ranging from 25 to $105 \mathrm{kHz}$, a range which covered most operating frequencies of the bat species represented in the analyzed data set. The chirps were recorded at a distance of 1 meter from the baffle with a measurement microphone (Brüel and Kjær, 1/8 inch). The microphone output was digitized at 16 bit resolution and $500 \mathrm{kHz}$ sampling rate (National Instruments NI pci-e 6351). The signals emitted through the baffles were recorded over an azimuth angular range of $176^{\circ}$ in steps of $1^{\circ}$ and an elevation angular range of $75^{\circ}$ in steps of $1^{\circ}$ to create a frontfacing beam pattern for the baffle. At each location a set of 20 chirps was recorded. A Fourier transform was applied to the chirps and the resulting frequency spectrum was converted to power. The resulting bin width of the power calculation was $500 \mathrm{~Hz}$, and five evenly spaced bins throughout the chirp were chosen for calculating beampatterns. At each azimuth and elevation point, the power of the 20 chirps were averaged within each frequency bin to create a front-facing beampattern at the five different frequencies. From the front-facing beampattern the beamwidth of the baffle was determined using the same method used to determine the beamwidth of the biosonar apertures.

\subsubsection{Creation of Random Emission Baffles}

There were five different emission baffles created from a piece of aluminum foil. The foil was first cut to size and them crumpled into a ball. The foil was uncrumpled into an oblong horn, similar in shape to biosonar apertures, before being mounted to the waveguide. By crumpling and uncrumpling the aluminum foil the interior geometry of the baffle was able to have entirely randomized crevices and veins that changed each test. The simulates had diameters ranging from $25 \mathrm{~mm}$ to $55 \mathrm{~mm}$ and were a mix of both elliptical baffles, with one diameter significantly larger than the other, and circular baffles with similar diameters. The length of the simulate, when measured from the waveguide to the edge of the simulate, ranged from $20 \mathrm{~mm}$ to $35 \mathrm{~mm}$. In order to help randomize the geometry of the baffles, three of the five baffles were created by other individuals to decrease any bias in their construction and shape. 


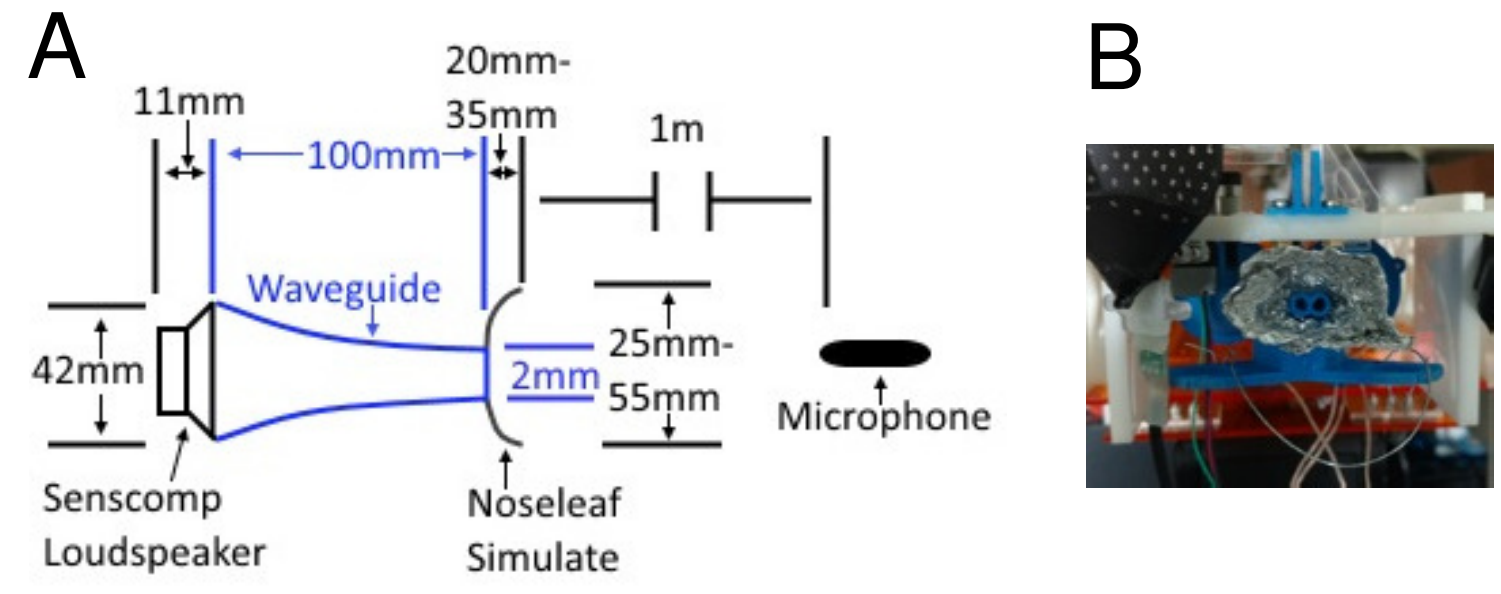

Figure 2.3: Setup for the experiment with the random aluminum baffles: A) Diagram of the experimental setup with dimensions of the loudspeaker, waveguide (shown in blue), and random aluminum foil baffles with the microphone in the far-field. B) Picture of the experiment set up. 


\section{Chapter 3}

\section{Results}

\subsection{Half-Power Beamwidths and Quarter-Power Beamwidths}

For bat biosonar, the half-power (HP) beamwidths tended to follow the theoretical limit closer than the quarter-power (QP) beamwidths, with the HP beamwidths having an average distance to the limit of $10^{\circ}$ and the QP beamwidths having an average distance to limit of $42^{\circ}$ (s. Figure 3.1). The HP beamwidths, however, had more values fall below the limit with $14 \%$ of the values below the limit compared to $1 \%$ of the QP beamwidths below the limit. The majority of the HP beamwidths below the limit were associated with comparatively low diameter to wavelength ratios (e.g., less than 4, s. Figure 3.1). Many of these biological baffle shapes created sidelobes in the beampatterns that extended up to the $-3 \mathrm{~dB}$ level but not the $-6 \mathrm{~dB}$ level.

Another difference between the half-power and quarter-power beamwidths was that in the region of lower array length to wavelength values the $\mathrm{QP}$ values increased compared to the limit more rapidly than the HP values (s. Figure 3.1). Overall, the results obtained with both amplitude threshold levels showed that the biosonar beamwidths did follow a tendency that matches the behavior of the theoretical beamwidth limit with the ratio of diameter to wavelength.

Comparing the beamwidth values obtained starting from the beam gain maximum (i.e., sidelobes excluded) with those starting from the periphery (i.e., sidelobes included) provided evidence for a role that sidelobes play in determining the beamwidth of the biosonar beampatterns. 14\% of the QP beamwidth values were affected by sidelobes with amplitudes that were large enough to effect either the maximum or the minimum beamwidth 

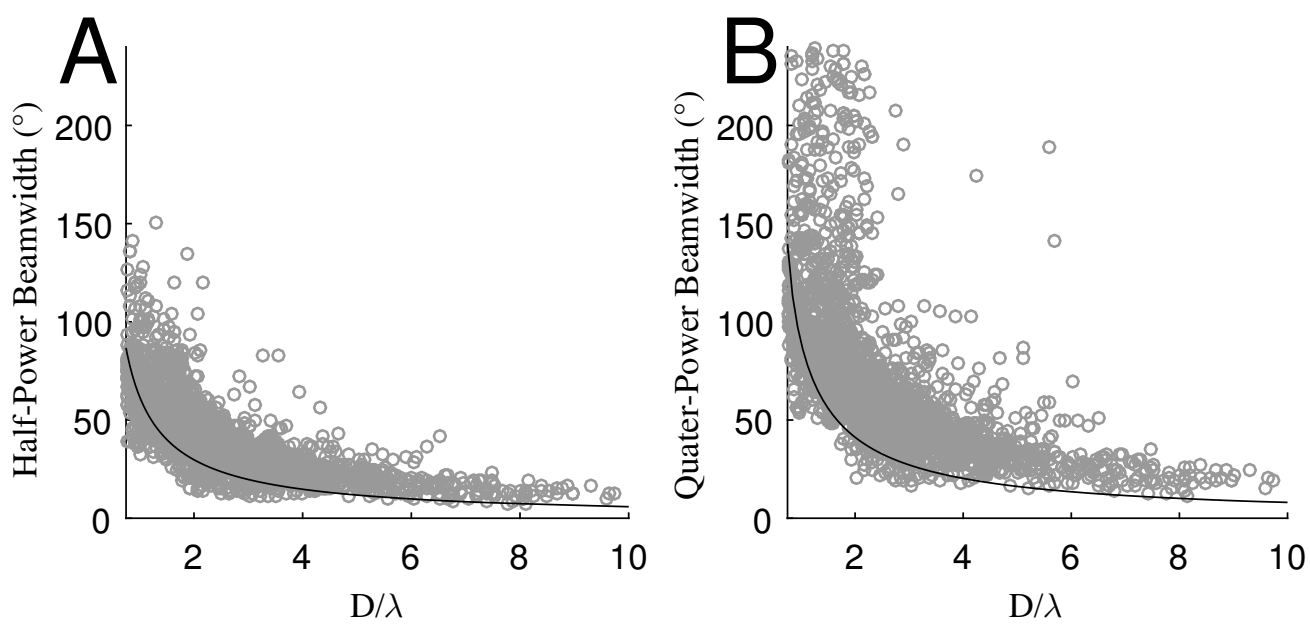

Figure 3.1: Comparison of half-power $(-3 \mathrm{~dB})$ beamwidth $(\mathrm{A})$ and quarter-power $(-6 \mathrm{~dB})$ beamwidth (B) for the bat biosonar elements. Theoretical minimum beamwidth values are shown as solid black lines for both levels. $D$ is the bat aperture diameter.

when included (s. Figure 3.2).

When examining the effect of sidelobes, a threshold of $10^{\circ}$ was used to determine whether a change in size was due to a sidelobe or ripple in the signal being unresolved. Sidelobes were more common in noseleaf beampatterns, in which $22 \%$ had sidelobes compared to $9 \%$ of the pinnae. The average increase in beamwidth due to sidelobes was $62^{\circ}\left( \pm 52^{\circ}\right.$ standard deviation). The data has a large standard deviation in part due to $20 \%$ of the sidelobes being very large $\left(>100^{\circ}\right)$.

The raw data for the half-power beamwidths excluding sidelobes is shown in Table 5.1 while the raw data for the quarter-power beamwidths excluding sidelobes is shown in Table 5.2. Both of these tables are located in Appendix B: Raw Data Tables. The raw data is provided so that so the analyses performed in this work can be duplicated as well as so that researchers interested in specific families, genera, or species can find information on the samples of particular interest.

When comparing sidelobes in half-power to sidelobes in quarter-power it was found that sidelobes had a larger impact in the QP region. 14\% of the QP beampatterns had significant sidelobes (s. Figure 3.4) compared to only $8 \%$ of the HP beamwidths (s. Figure 3.3). In the $\mathrm{HP}$ beamwidths the average increase in sidelobe was also smaller at $44^{\circ}\left( \pm 31^{\circ}\right.$ standard deviation) and had a smaller standard deviation due to only $6 \%$ of the sidelobes being very large $\left(>100^{\circ}\right)$. However, another distinction between the two beamwidths is that the QP 


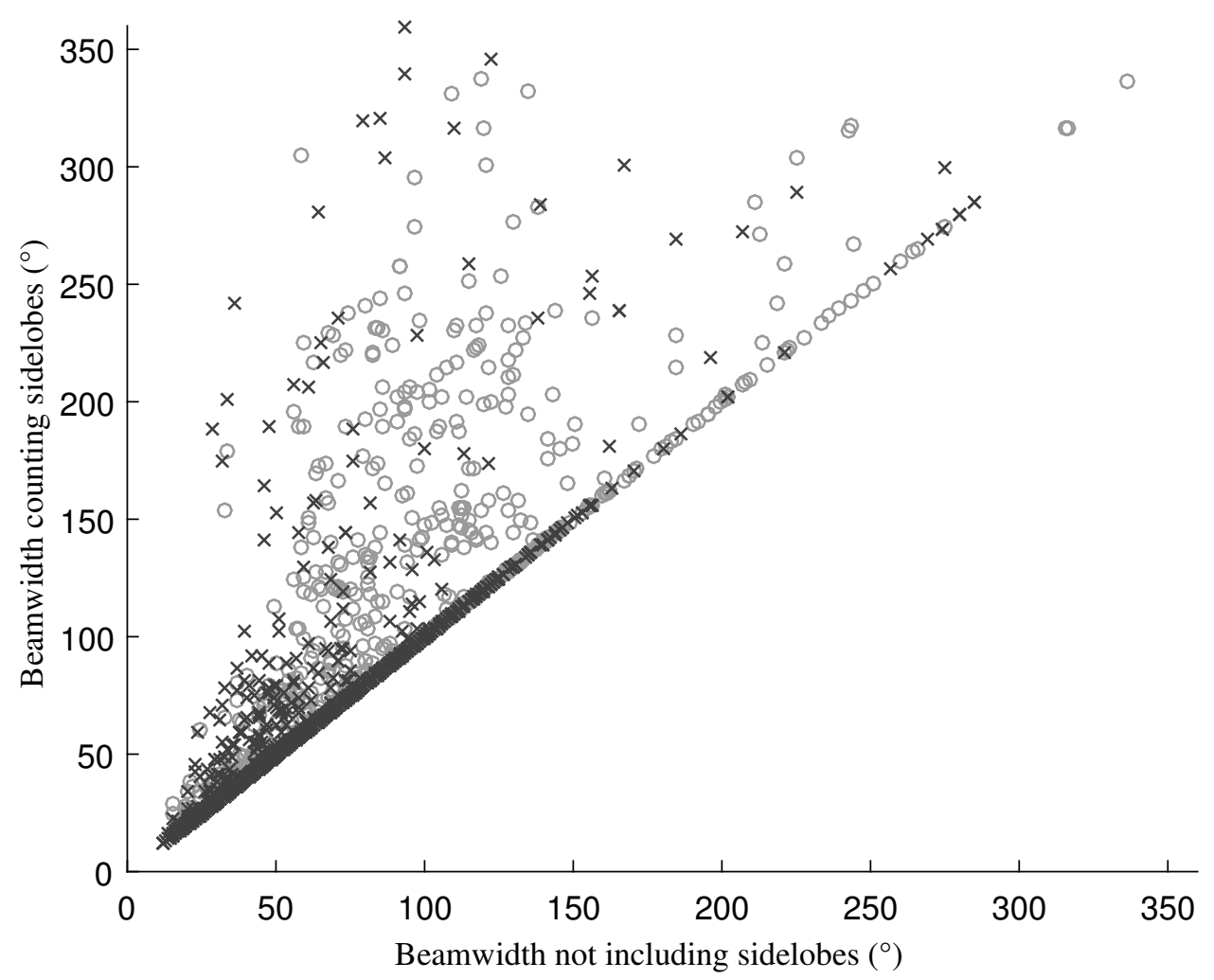

Figure 3.2: Impact of sidelobes on the beamwidth of the biosonar beampattern samples for noseleaves (gray circles) and pinnae (black x's). Points on the diagonal line do not have sidelobes amplitudes that are large enough to effect the beamwidth estimates. 

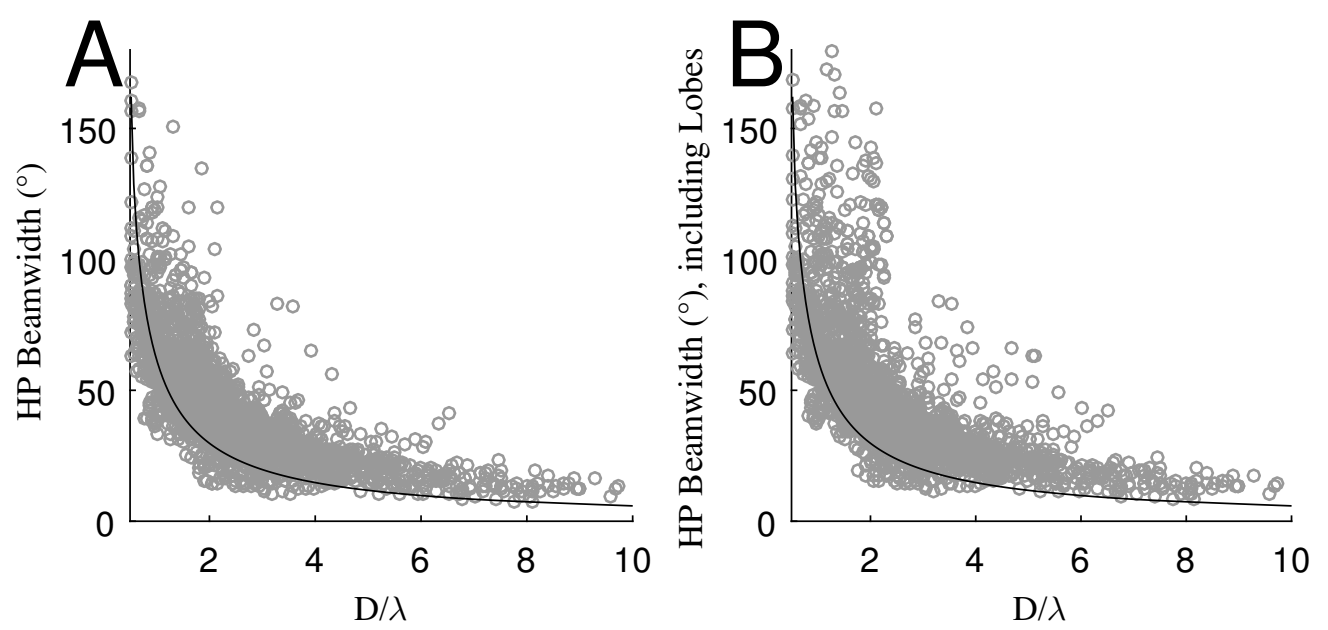

Figure 3.3: Half-power beamwidth values with respect to the aperture diameter $(D)$ to wavelength $(\lambda)$ ratio. A) The HP beamwidths calculated using the first point where the beampattern amplitudes crossed the HP threshold and B) the HP beamwidths calculated using the furthest point where the beampattern crossed the HP threshold.

beamwidths had ripple in the signal around the threshold resulting in small changes to the beamwidth in $6 \%$ of the samples while the HP beamwidths had similar ripple in $15 \%$ of beamwidths.

\subsection{Beamwidth Comparison of Engineered and Biosonar}

The HP beamwidths of the biosonar and engineered sonar samples both showed a tendency similar to the theoretical beamwidth limit, in that larger ratios of array-length to wavelength tended to produce smaller beamwidths. However, the majority of the engineered sonars were located in a region of much higher array-length to wavelength ratios than the biosonars. In the engineered sonars, the beamwidth stayed very close to the theoretical limit, especially for systems with larger ratios of array-length to wavelength. In contrast to this, the biosonar samples showed a much larger scatter away from the theoretical limit curve in the direction towards larger beamwidths (s. Figure 3.5).

While it can be seen from Figure 3.5 that the biosonar apertures appear to scatter further from the reference limit than the technical sonars do, an analysis of variance (ANOVA) test was conducted in order to determine if this difference was statistically significant. The 

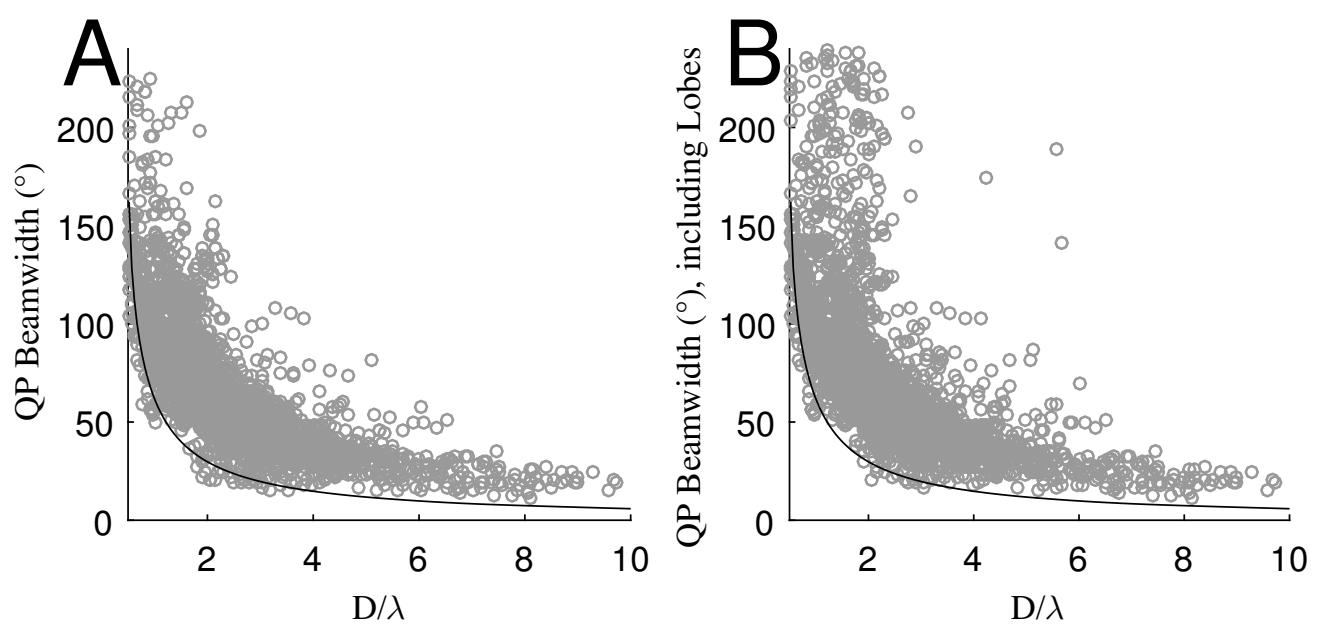

Figure 3.4: Quarter-power beamwidth values with respect to the aperture diameter $(D)$ to wavelength $(\lambda)$ ratio. A) The QP beamwidths calculated using the first point where the beampattern amplitudes crossed the QP threshold and B) the QP beamwidths calculated using the furthest point where the beampattern crossed the QP threshold.

null hypothesis for the ANOVA test was that the biosonar and engineered sonar had similar scatter from the limit, and the scatter from the distance was calculated using the half-power beamwidths. The $\mathrm{p}$-value for the test was found to be significantly smaller than a 0.01 significance level (s. Table 3.1), resulting in rejecting the null hypothesis. Additionally, the mean value of the scatter of the biosonar was significantly larger than the mean scatter of the engineered sonar (s. Figure 3.6). These results corroborate the inspection of the graph, showing the increased scatter of the biosonar is statistically significant.

Table 3.1: Results of the analysis of variance test comparing the scatter from the limit of the biosonar apertures and the engineered sonars. All comparisons were performed on the half-power beamwidth data. SS is the sum of squares, df is the degrees of freedom, MS is the mean squared error and $\mathrm{F}$ is the $\mathrm{f}$ statistic, which is the ratio of the mean squared error of the groups to the mean squared error of the error.

\begin{tabular}{cccccc} 
Source & SS & df & MS & F & Prob $>$ F \\
\hline Groups & 2419.1 & 1 & 2419.07 & 21.4 & $3.89 \times 10^{-6}$ \\
Error & 308102.8 & 2726 & 113.02 & & \\
Total & 310521.8 & 2727 & & &
\end{tabular}




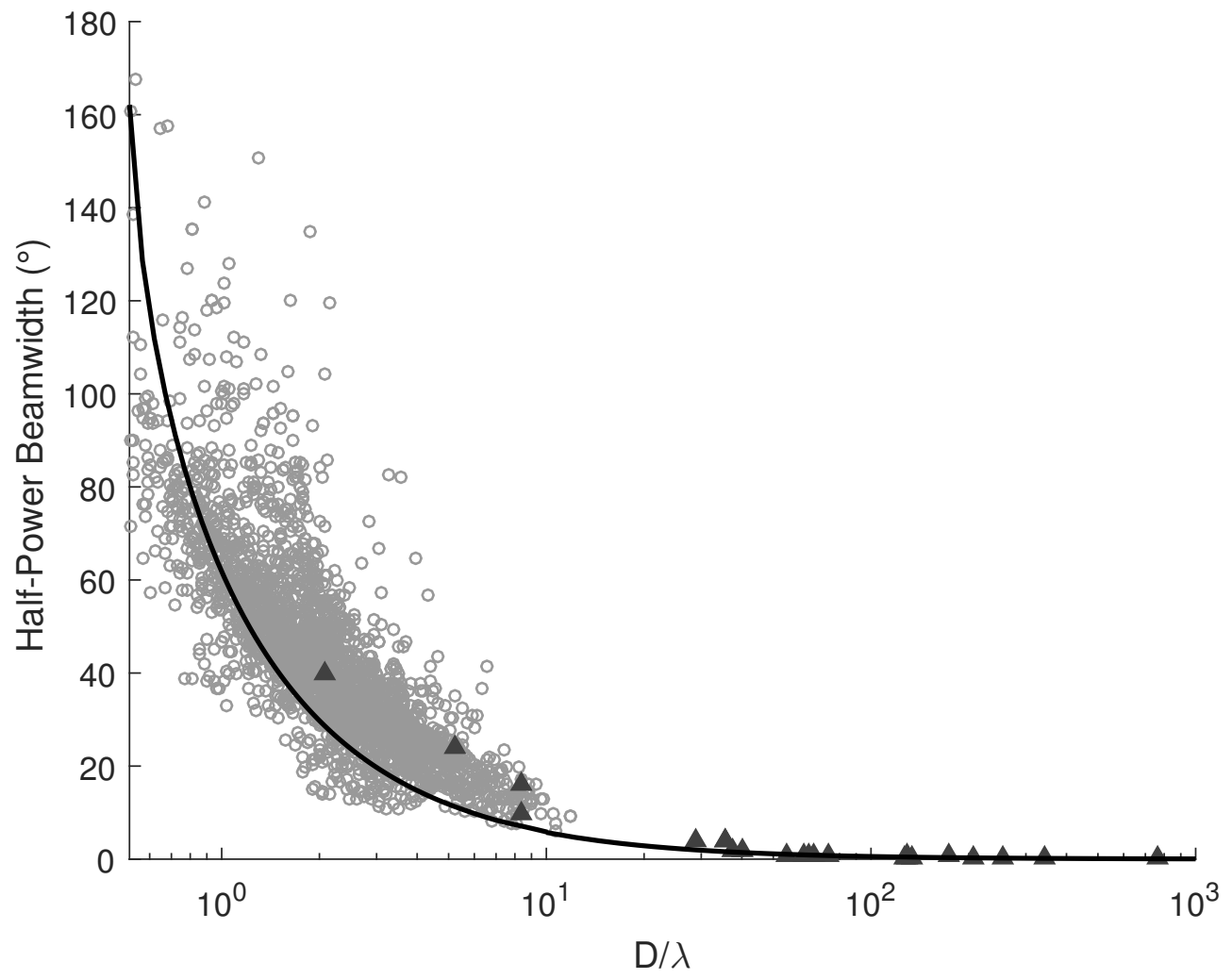

Figure 3.5: Comparison of biosonar and engineered sonar half-power beamwidths to the elliptical-transducer limit values (solid line) as a function of the ratio characteristic dimension $(D)$ over wavelength $(\lambda)$. Biosonar samples are represented by light gray circles and engineered sonars by black triangles. $D$ is a characteristic dimension of the sonar, i.e., array-length for technical sonar and noseleaf or pinna diameter for bat biosonar. 


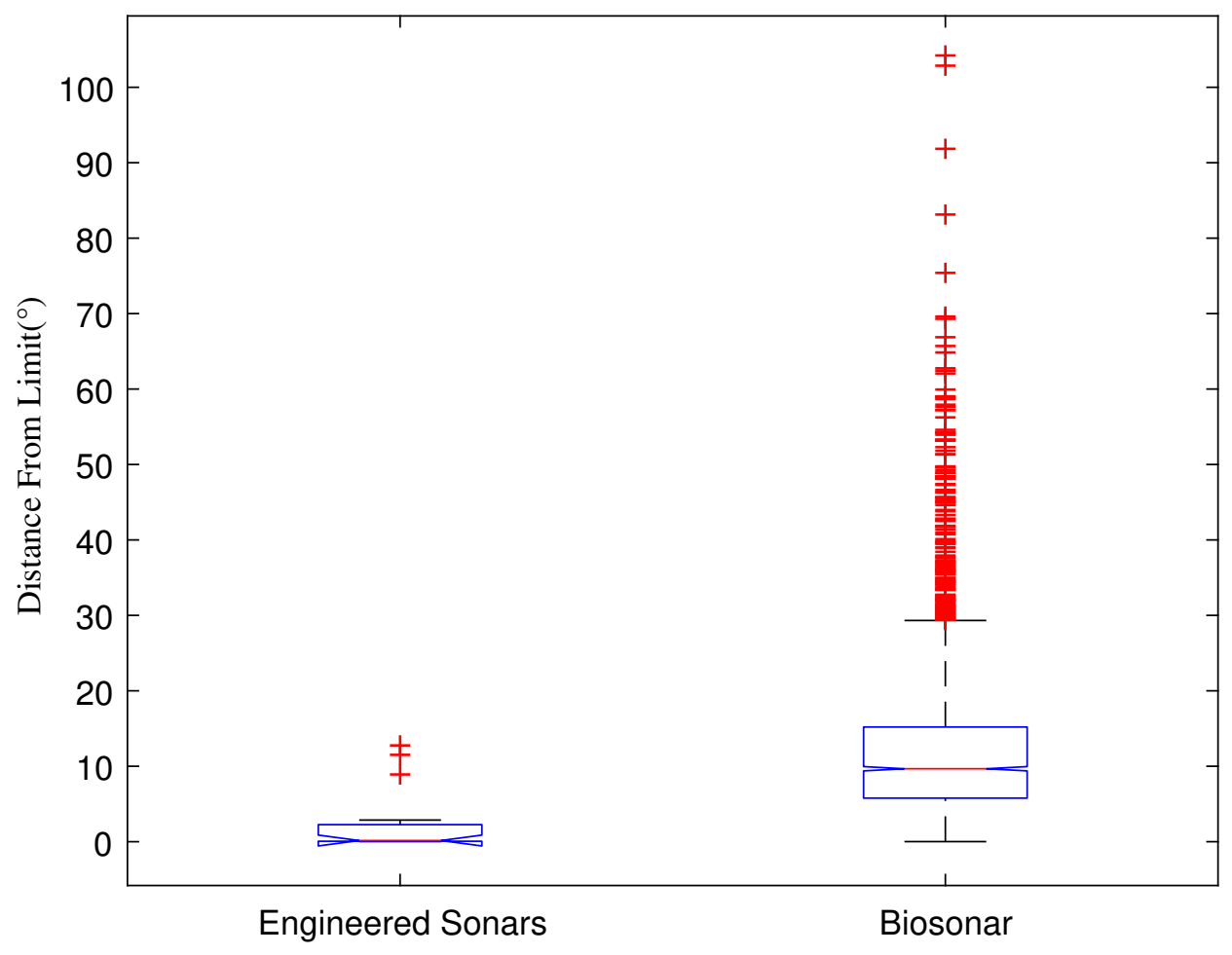

Figure 3.6: Results of the analysis of variance test comparing the scatter of the engineered sonars and biosonars to the reference limit at half-power. For each set of data, the red line inside of the blue box indicates the mean of that set, with the upper and lower edges of the blue box indicating the first and third quantiles. The red crosses represent data points considered outliers within the set. 


\subsection{Noseleaf Simulate Experiment Results Compared to Biosonar}

The experimental beamwidth results obtained from the noseleaf simulate baffles showed a similar scatter away from the elliptical-transducer limit as the bat samples (s. Figure 3.7). The scatter associated with the biological samples appeared to be somewhat bigger than that associated with the aluminum baffles, especially towards lower values of the ratio aperture diameter $(D)$ to wavelength $(\lambda)$ where most of the experimental data points were concentrated. For ratios around the lower end of this region, the biological samples appeared to show a larger variability than the random shapes, but it is not clear if this is a genuine trend in the data or just an effect of the small sample size.

An ANOVA test was performed to determine if there were statistically significant differences between the scatter of the biosonar and the scatter of the noseleaf simulates. The null hypothesis to be tested was that the biosonar and noseleaf simulates had similar scatter. The scatter from the reference limit for both sets was calculated at quarter-power, where they were compared in Figure 3.7. The p-value of the ANOVA test results was found to be significantly smaller than a significance value of 0.01 , causing the null hypothesis to be rejected (s. Table 3.2). By looking at the results of the ANOVA test it can be seen that the noseleaf simulates have a lower mean scatter (s. Figure 3.8). This indicates that the biosonar have statistically significant additional scatter from the reference limit compared to the noseleaf simulates.

Table 3.2: Results of the analysis of variance test comparing the scatter from the limit of the biosonar apertures and the noseleaf simulates. All comparisons were performed on the quarter-power beamwidth data. SS is the sum of squares, df is the degrees of freedom, MS is the mean squared error and $\mathrm{F}$ is the $\mathrm{f}$ statistic, which is the ratio of the mean squared error of the groups to the mean squared error of the error.

\begin{tabular}{lrrrll} 
Source & \multicolumn{1}{l}{ SS } & df & \multicolumn{1}{l}{ MS } & F & Prob $>$ F \\
\hline Groups & 72981.7 & 1 & 72981.7 & 122.09 & $8.29 \times 10^{-28}$ \\
Error & 1662421 & 2781 & 597.8 & & \\
Total & 1735402 & 2782 & & &
\end{tabular}




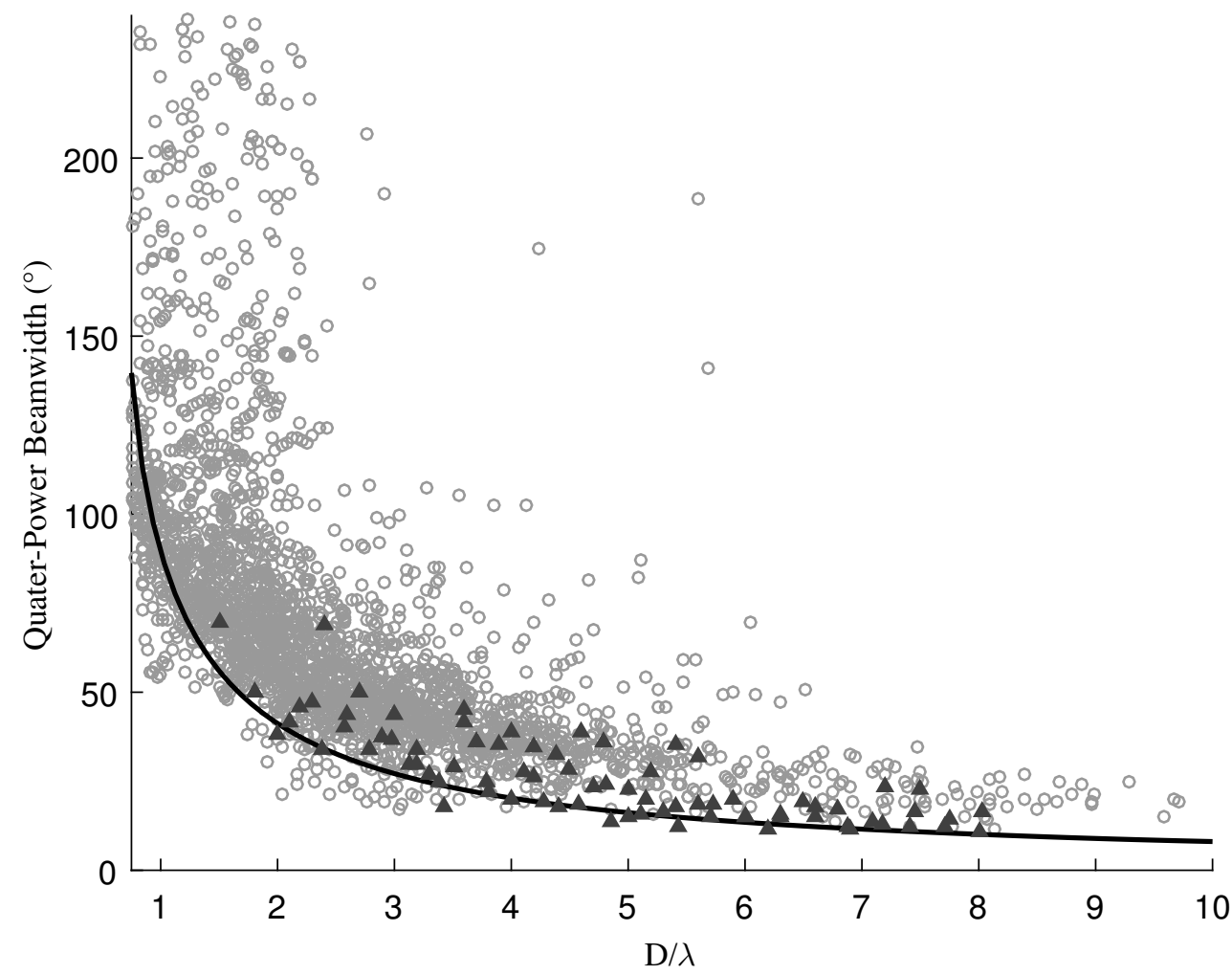

Figure 3.7: Comparison of the beamwidths of the biological samples with randomly shaped aluminum baffles as a function of the ratio aperture diameter over wavelength $\left(\frac{D}{\lambda}\right)$. Biological samples are shown as gray circles, aluminum baffles as black triangles. 


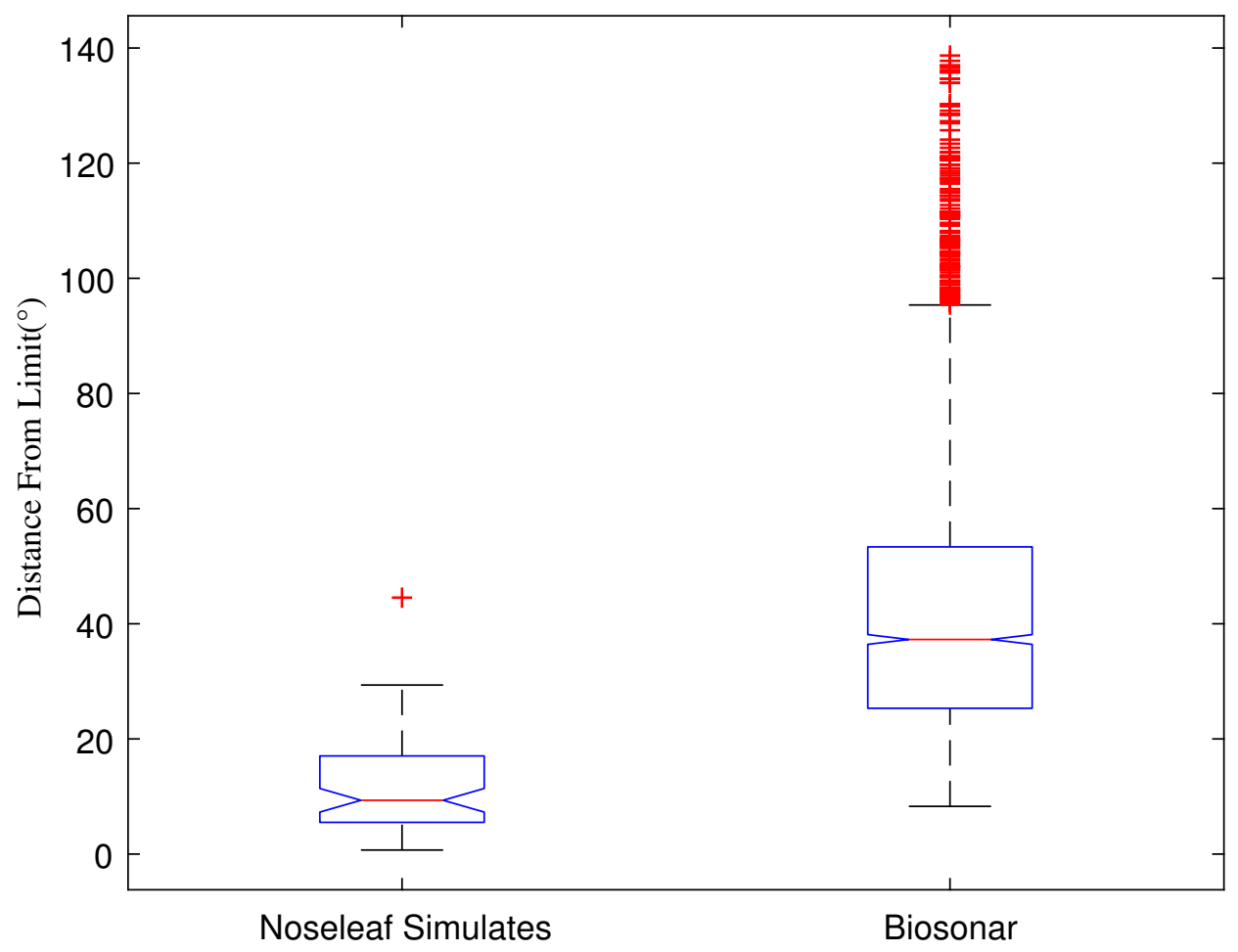

Figure 3.8: Plot results of the analysis of variance test comparing the scatter of the noseleaf simulates and biosonars to the reference limit at quarter-power. For each set of data, the red line inside of the blue box indicates the mean of that set, with the upper and lower edges of the blue box indicating the first and third quantiles. The red crosses represent data points considered outliers within the set. 

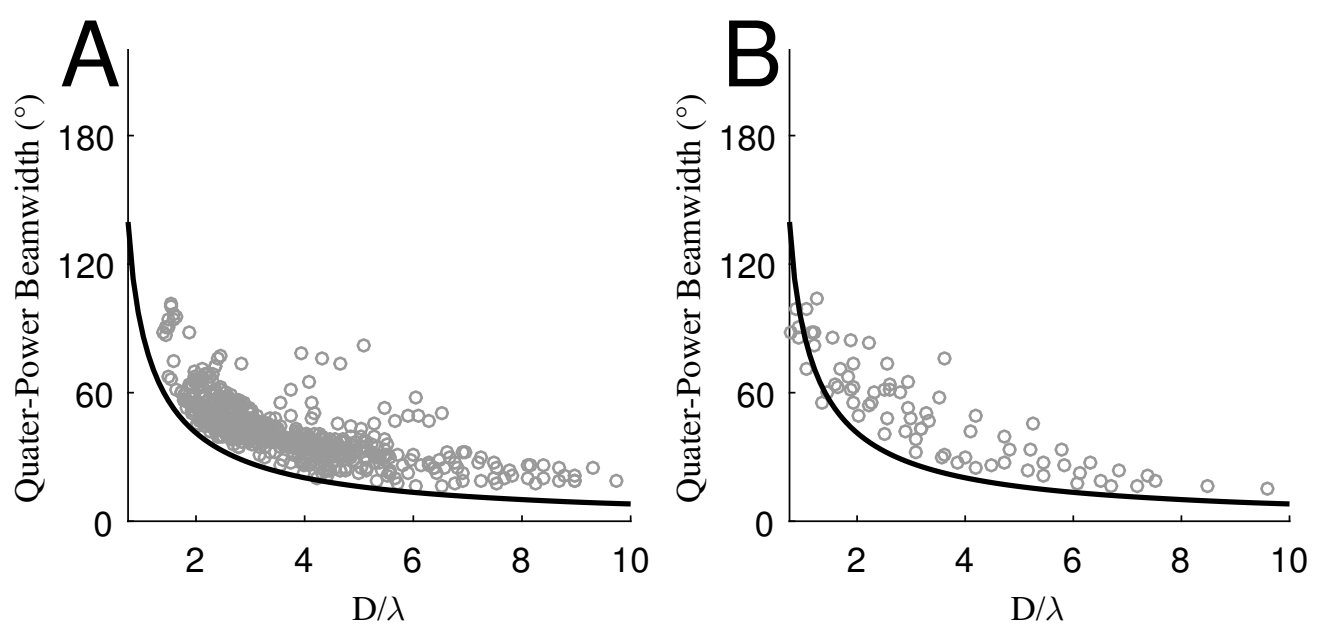

Figure 3.9: Comparison of beamwidths in two bat families with heavy reliance on active biosonar (horseshoe bats, Rhinolophidae, and Old World roundleaf bats, Hipposideridae, A) and a bat family where the use of active biosonar only occurs as an exception in a few species (Old World fruit bats, Pteropodidae, B). For both families, the QP beamwidth associated with the samples as a function of the ratio of aperture diameter $(D)$ to wavelength $(\lambda)$ are shown along with the elliptical-transducer limit.

\subsection{Comparison of Bat Families With and Without Active Biosonar}

The families of horseshoe bats (Rhinolophidae) and the Old World roundleaf bats (Hipposideridae) are known to rely on particularly sophisticated active sonar systems that enable them to navigate and hunt in dense vegetation [13]. As these two families of bats are known to use and rely on sonar systems, they can be used as a basis to compare to a family of bats (Old World fruit bats, family Pteropodidae) that - with few exceptions - do not use active biosonar [13]. These two families were compared, using only pinnae as the Old world fruit bats do not have noseleaves, to find that even though the horseshoe bats and roundleaf bats use a very sophisticated active sonar system they still show the same trends to beamwidth as a family that does not use active sonar (s. Figure 3.9).

To verify that were no statistically significant differences in the scatter from the reference limit of the echolocating and non-echolocating families an ANOVA test was performed. The null hypothesis for the ANOVA test was set as the echolocating and non-echolocating families having equal scatter from the reference limit, measured in quarter-power. The p- 
value was significantly larger than a significance value of 0.01 , causing the null hypothesis to fail to be rejected (s. Table 3.3). Looking at the ANOVA plot it can be seen that the means of the two sets are roughly equivilent though the non-echolocating families have a larger range of the first and third quantile (s. Figure 3.10). This difference in quantile position could be due to having fewer samples from the non-echolocating families.

Table 3.3: Results of the analysis of variance test comparing the scatter from the limit of the echolocating families (Rhinolophidae and Hipposideridae) and the non-echolocating family (Pteropodidae). All comparisons were performed on the quarter-power beamwidth data. SS is the sum of squares, $\mathrm{df}$ is the degrees of freedom, MS is the mean squared error and $\mathrm{F}$ is the $\mathrm{f}$ statistic, which is the ratio of the mean squared error of the groups to the mean squared error of the error.

\begin{tabular}{lrrrlr} 
Source & \multicolumn{1}{l}{ SS } & \multicolumn{1}{l}{ df } & \multicolumn{1}{c}{ MS } & F & Prob $>$ F \\
\hline Groups & 30.8 & 1 & 30.79 & 0.36 & 0.5484 \\
Error & 66457.5 & 778 & 85.421 & & \\
Total & 66488.3 & 779 & & &
\end{tabular}

\subsection{Emission vs Reception Elements}

Comparing the beamwidths belonging to pinna samples to those obtained from noseleaves showed two main differences. First, noseleaf samples tended to be smaller compared to their respective operating wavelengths than the pinnae; for the noseleaves, $63 \%$ of the samples had a diameter-to-wavelength ratio less than 2 . For the pinnae, this was the case for $40 \%$ of the samples (s. Figure 3.11). Second, the noseleaves tended to deviate further from the theoretical limit values. The noseleaf beamwidths deviated from the theoretical limit by $15^{\circ}$ on average (standard deviation $35^{\circ}$ ) compared to $5^{\circ}$ average distance (standard deviation $23^{\circ}$ ) for pinnae (s. Figure 3.12).

In Figure 3.12, and based on the above calculations of mean and standard deviation, it appears that there are significant differences between emission and reception elements in the biosonar. In order to test whether these differences were statistically significant, an ANOVA test was performed. The null hypothesis for the test was set that the emission and reception elements had similar scatter from the reference limit. The scatter was calculated in quarter power and only for values of $D / \lambda$ less than 4 where the majority of the noseleafs were located. The p-value was significantly smaller than a significane value of 0.01 , causing the null hypothesis to be rejected (s. Table 3.4). Upon inspection of the ANOVA 


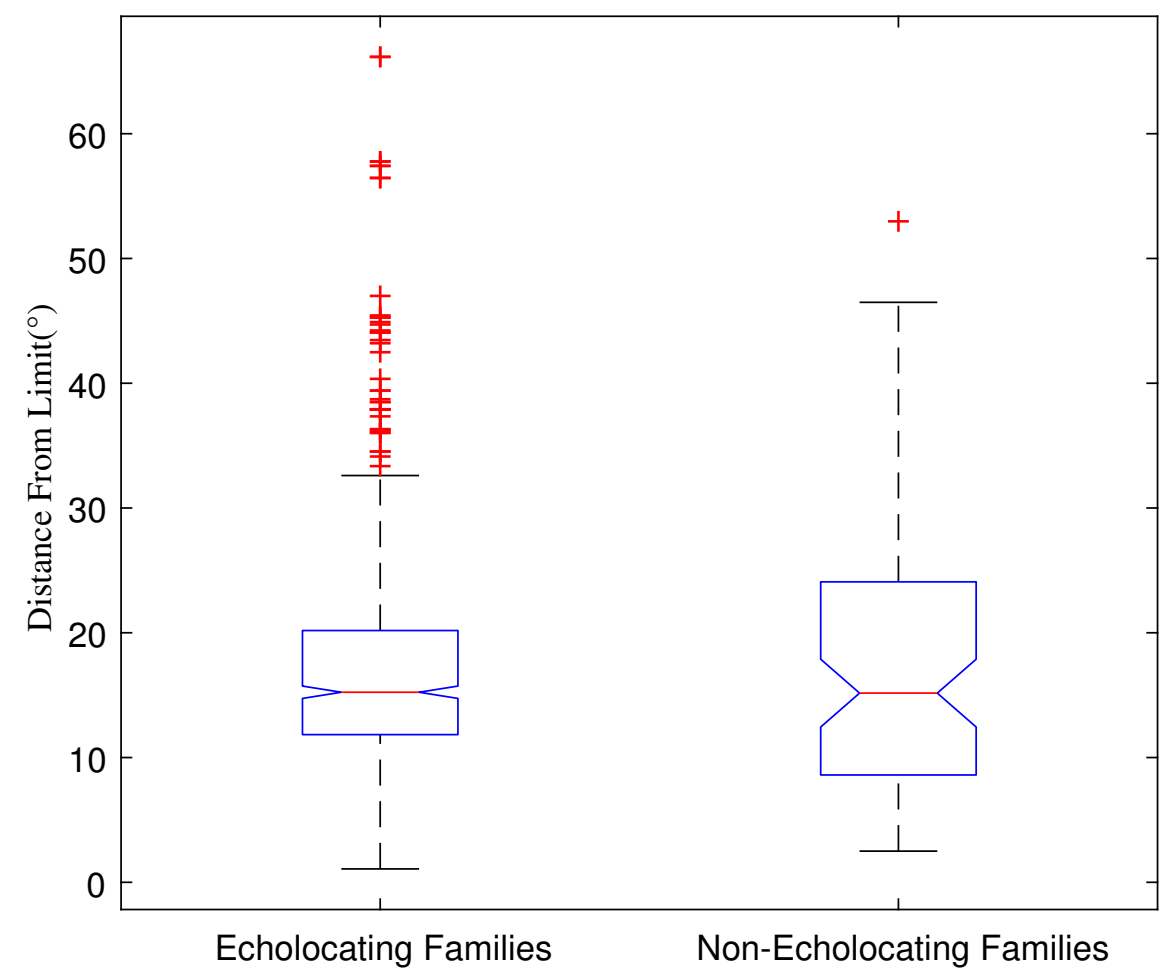

Figure 3.10: Results of the analysis of variance test comparing the scatter of the echolocating families (Rhinolophidae and Hipposideridae) and non-echolocating family (Pteropodidae) to the reference limit at quarter-power. For each set of data, the red line inside of the blue box indicates the mean of that set, with the upper and lower edges of the blue box indicating the first and third quantiles. The red crosses represent data points considered outliers within the set. 


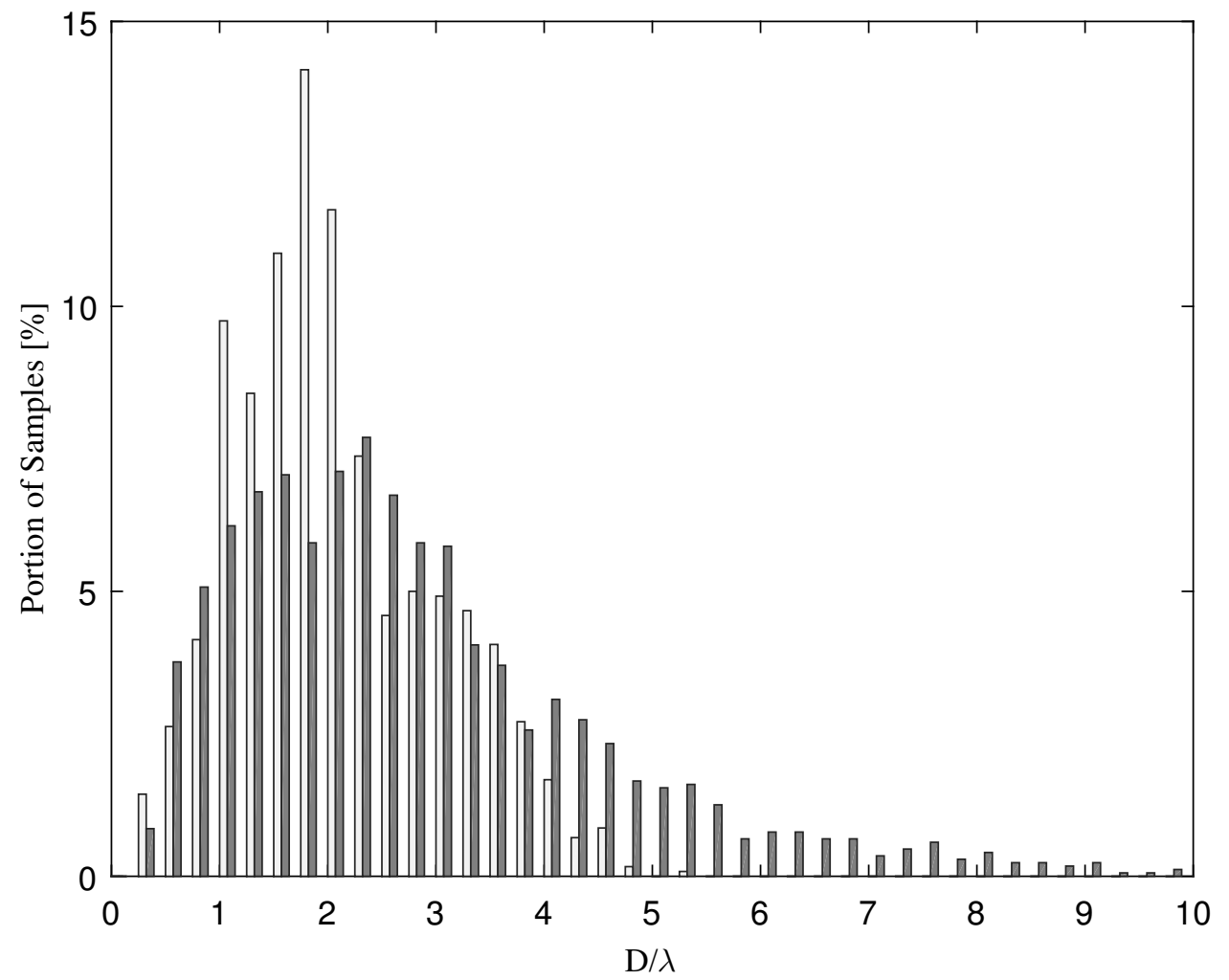

Figure 3.11: Comparison of the distributions for noseleaf (light gray) and pinna samples (dark gray) with respect to the ratio aperture diameter $(D)$ over wavelength $(\lambda)$. 

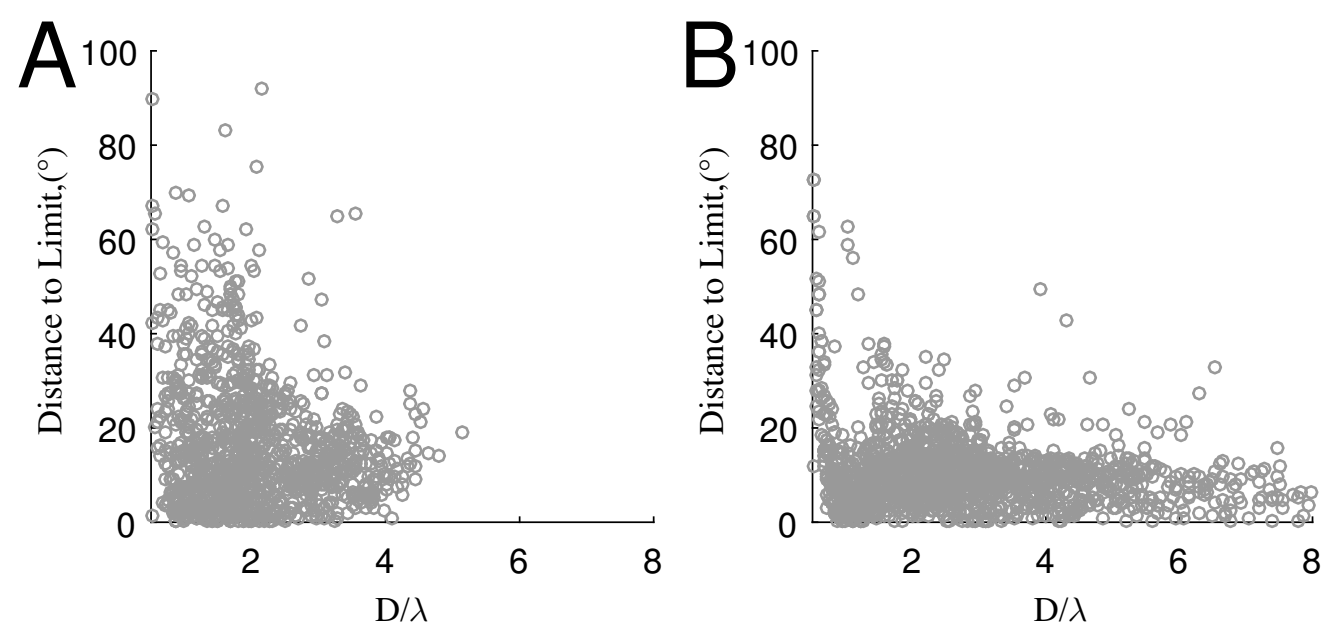

Figure 3.12: Distance between the beamwidth values of the biosonar samples and the respective lower beamwidth limit as a function of the ratio of characteristic dimension $(D)$ over wavelength $(\lambda)$. A) noseleaf samples, B) pinna samples. $D$ is a characteristic dimension, i.e., aperture diameter for the bat biosonar samples.

plot it is seen that the pinnae have a smaller mean scatter and smaller sizes of the quantiles (s. Figure 3.13).

\subsection{Additional Supporting Analysis}

The following sets of analysis did not have as strong results as those in previous sections, however the results still contain some insights into how biosonars operate and how they are potentially different from engineered sonar.

\subsubsection{Maximum vs Minimum Beamwidth}

The minimum and maximum beamwidths, matched with the maximum and minimum aperture sizes respectively, can be compared to see that the minimum beamwidths both occupy a much larger range of $D / \lambda$ values and over that range they tend to stay much closer to the limit. The maximum beamwidths were all found below a $D / \lambda$ of 7 and $78 \%$ were found to have a $D / \lambda$ of 3 or lower. In the half-power regime, it was found that the matched maximum beamwidths were on average $21^{\circ}$ larger $\left( \pm 15^{\circ}\right.$ standard deviation $)$, 


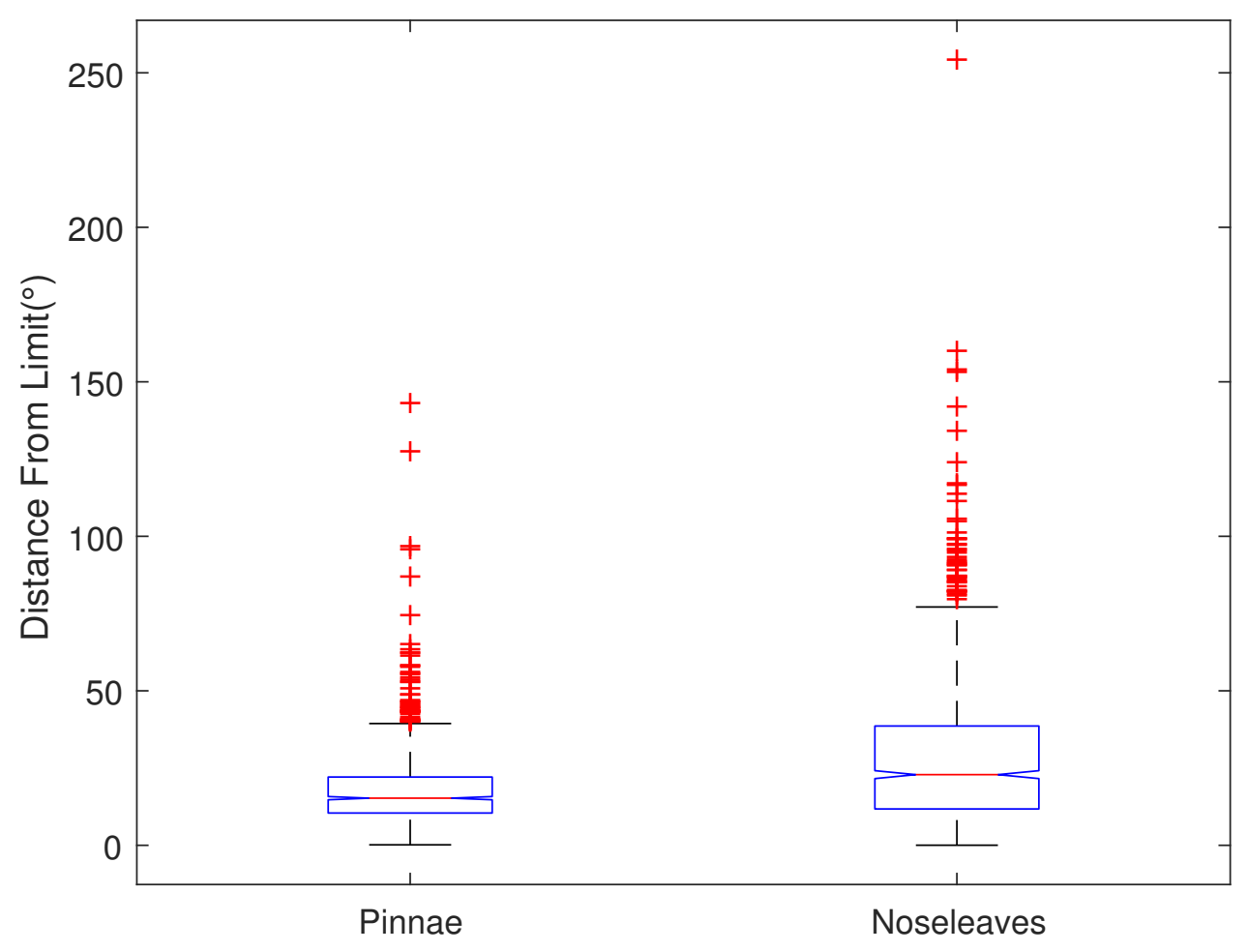

Figure 3.13: Results of the analysis of variance test comparing the scatter of the reception elements (pinnae) and emission elements (noseleaves) to the reference limit at quarterpower. The comparison was performed only for values of $D / \lambda$ less than 4 to keep the comparison in the regime where noseleaves were typically located. For each set of data, the red line inside of the blue box indicates the mean of that set, with the upper and lower edges of the blue box indicating the first and third quantiles. The red crosses represent data points considered outliers within the set. 
Table 3.4: Results of the analysis of variance test comparing the scatter of the reception elements (pinnae) and emission elements (noseleaves). All comparisons were performed on the quarter-power beamwidth data. The comparison was performed only for values of $D / \lambda$ less than 4 to keep the comparison in the regime where noseleaves were typically located. SS is the sum of squares, $\mathrm{df}$ is the degrees of freedom, MS is the mean squared error and $\mathrm{F}$ is the $\mathrm{f}$ statistic, which is the ratio of the mean squared error of the groups to the mean squared error of the error.

\begin{tabular}{lrrrll} 
Source & \multicolumn{1}{l}{ SS } & df & \multicolumn{1}{l}{ MS } & F & Prob $>$ F \\
\hline Groups & 73494.9 & 1 & 73494.9 & 205.85 & $8.71 \times 10^{-45}$ \\
Error & 825440.8 & 2312 & 357 & & \\
Total & 898935.7 & 2313 & & &
\end{tabular}

while in the quarter-power beamwidths the average difference was $47^{\circ}$ ( $\pm 47^{\circ}$ standard deviation). Pinnae in half-power had an average difference of $15^{\circ}$ compared to noseleaves with an average difference of $27^{\circ}$.

\subsubsection{Geometric Effects on Beamwidth}

Two different methods were used to test the effect of geometric factors on the accuracy of the reference model to the calculated beamwidths. The first comparison checked how well the apertures could be evaluated as 2 dimensional by calculating the root mean square distance traveled by the point clouds to the plane of best fit in order to determine the depth of the aperture (s. Figure 3.15). After comparison the depth of the aperture did not appear to have any noticeable affect or trend on the likelihood of the beamwidth falling closer to the reference limit.

Another property examined was the eccentricity, i.e. roundness, of the $2 \mathrm{D}$ transducer. Many of the biosonar apertures were much more elliptical, and similarly the eccentricity was measured and then plotted to look for trends based on location on the beamwidth curve (s. Figure 3.16). The eccentricity did not appear to effect how close a specific value fell towards the curve and there were not any apparent trends of eccentricity that were noted from the curve.

In circular plane arrays and engineered transducers there is an approximately linear relationship between the ratio of the minimum and maximum diameters of the array or aperture and the minimum and maximum beamwidth. This relationship was compared for the biosonar samples. For each of the biosonar samples, the ratio of minimum to maximum 

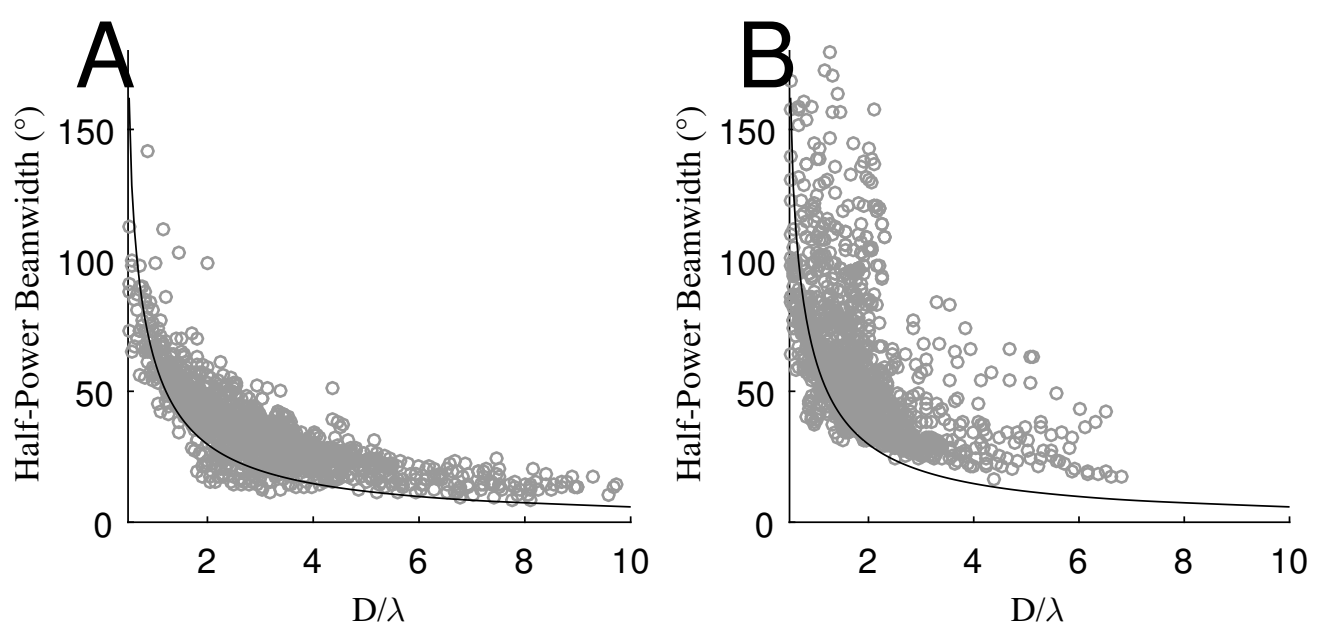

Figure 3.14: Comparison of minimum and maximum beamwidths: A) Minimum beamwidth values matched with the maximum aperture diameters. B) Maximum beamwidth values matched with the minimum aperture diameters.

beamwidth was averaged over the range of operating frequencies to compare to the technical reference (s. Figure 3.17). The averages were found to be scattered around the line of expected behavior, indicating that the biosonars do not follow the expected curve for eccentricity of geometry compared to eccentricity of beamwidth. Additionally, for a given aperture over the ten operating frequencies that beampatterns were generated for, the eccentricity of the beamwidth had an average increase of .21 eccentricity between the most elliptical and most circular of the beampatterns. These results indicate that the complex geometries of the biosonar apertures do not allow approximation of one beamwidth when given the other, unlike in elliptical transducers.

\subsubsection{Distance To Limit}

In the biosonar samples, the difference between the beamwidth values of the samples and the respective elliptical-transducer limit values was found to depend on the ratio between diameter and wavelength. In absolute terms, this difference decreased from about $15^{\circ}$ to about $5^{\circ}$, as the ratio diameter over wavelength increased, i.e., the larger the noseleaves or pinnae were compared to the wavelengths they operate on, the closer their beamwidths came to the limit (s. Figure 3.18). 


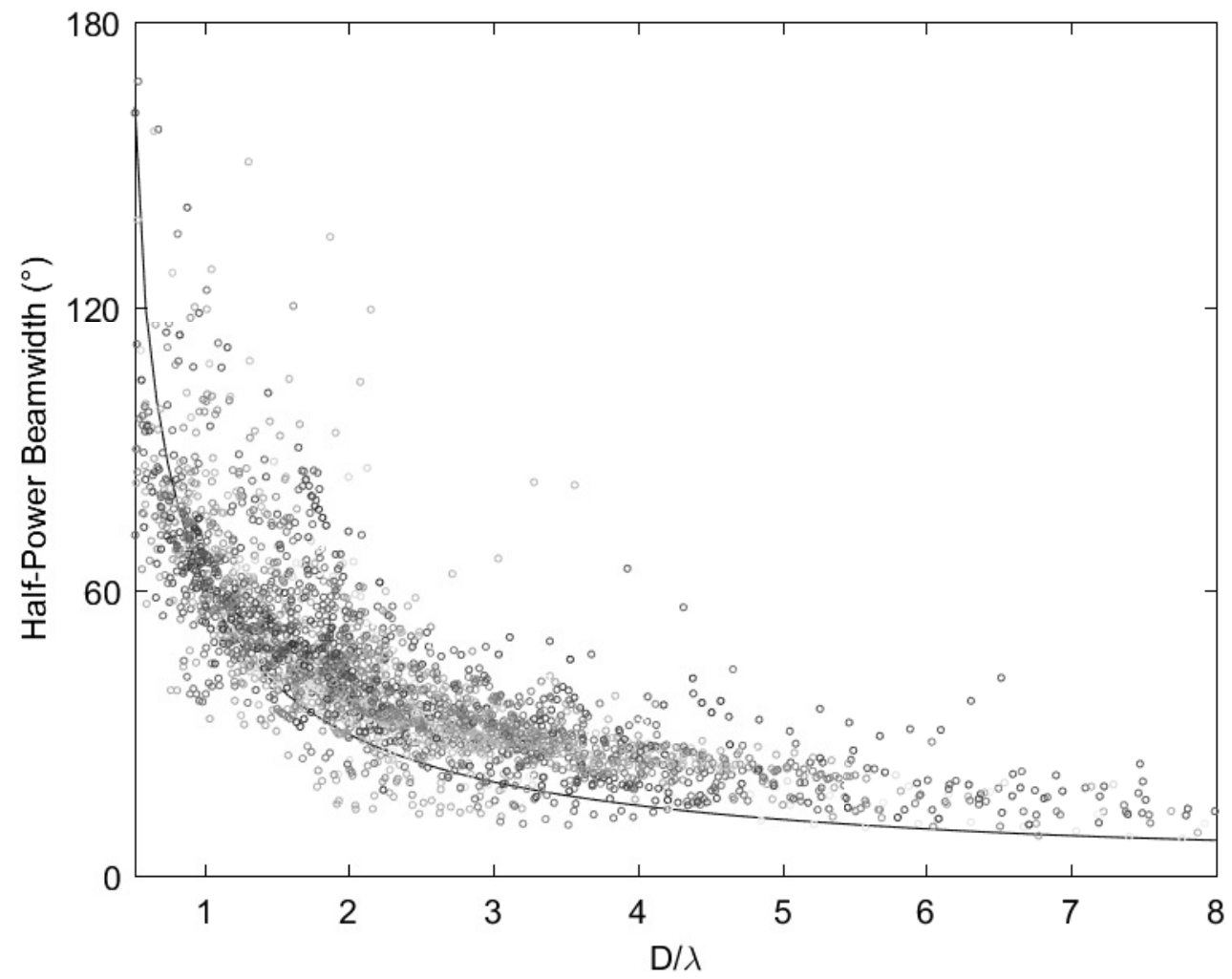

Figure 3.15: The reference beamwidth values is plotted as the black line with respect to aperture diameter $(D)$ to wavelength $(\lambda)$. The scattered points are on a grey scale, where the darker a point is, the flatter the aperture is. 


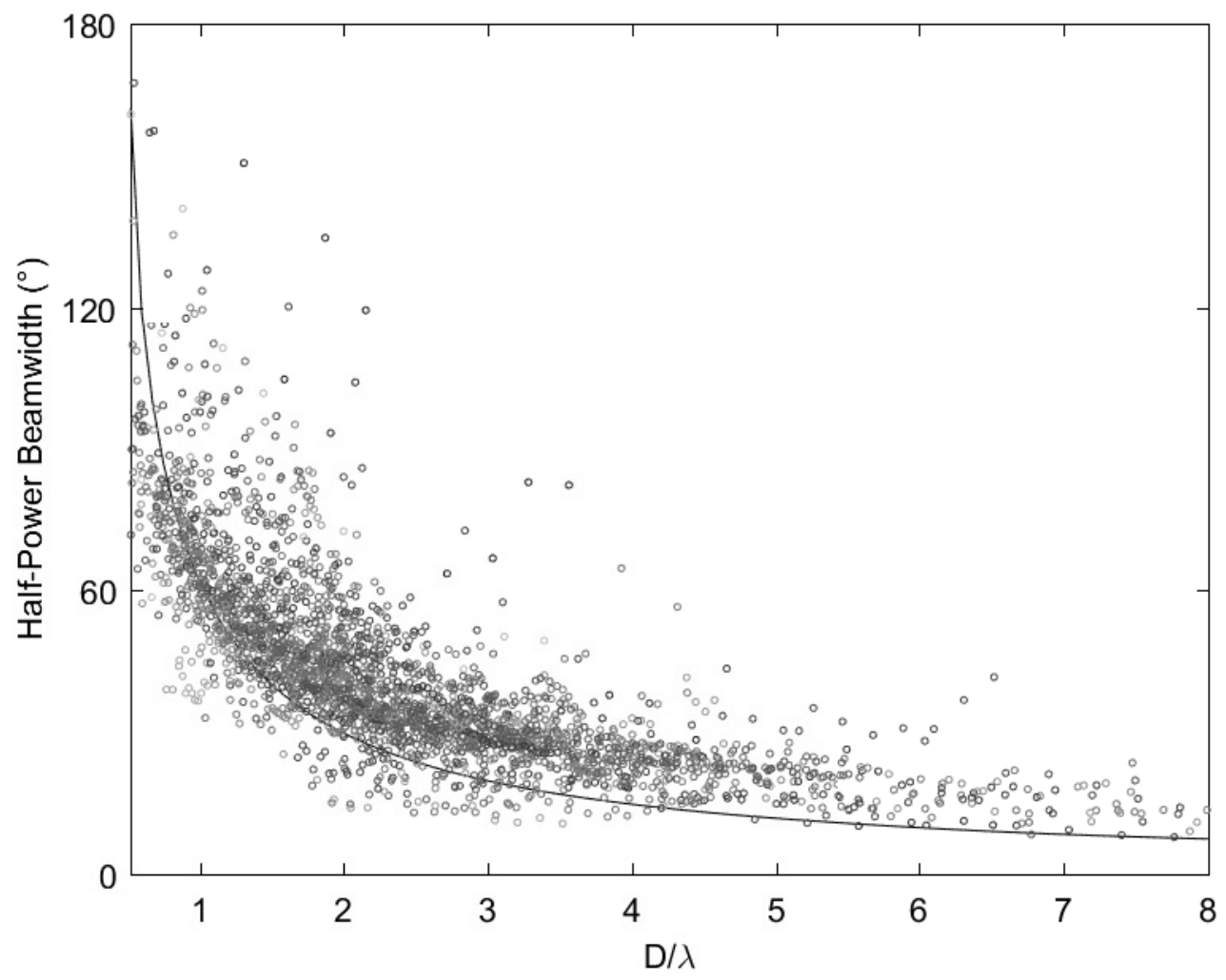

Figure 3.16: The reference beamwidth value is plotted as the black line with respect to aperture diameter $(D)$ to wavelength $(\lambda)$. Eccentricity is measured by the greyscale of the points on the graph, where the darker a point is, the lower it's eccentricity, i.e. the less circular the aperture is. 


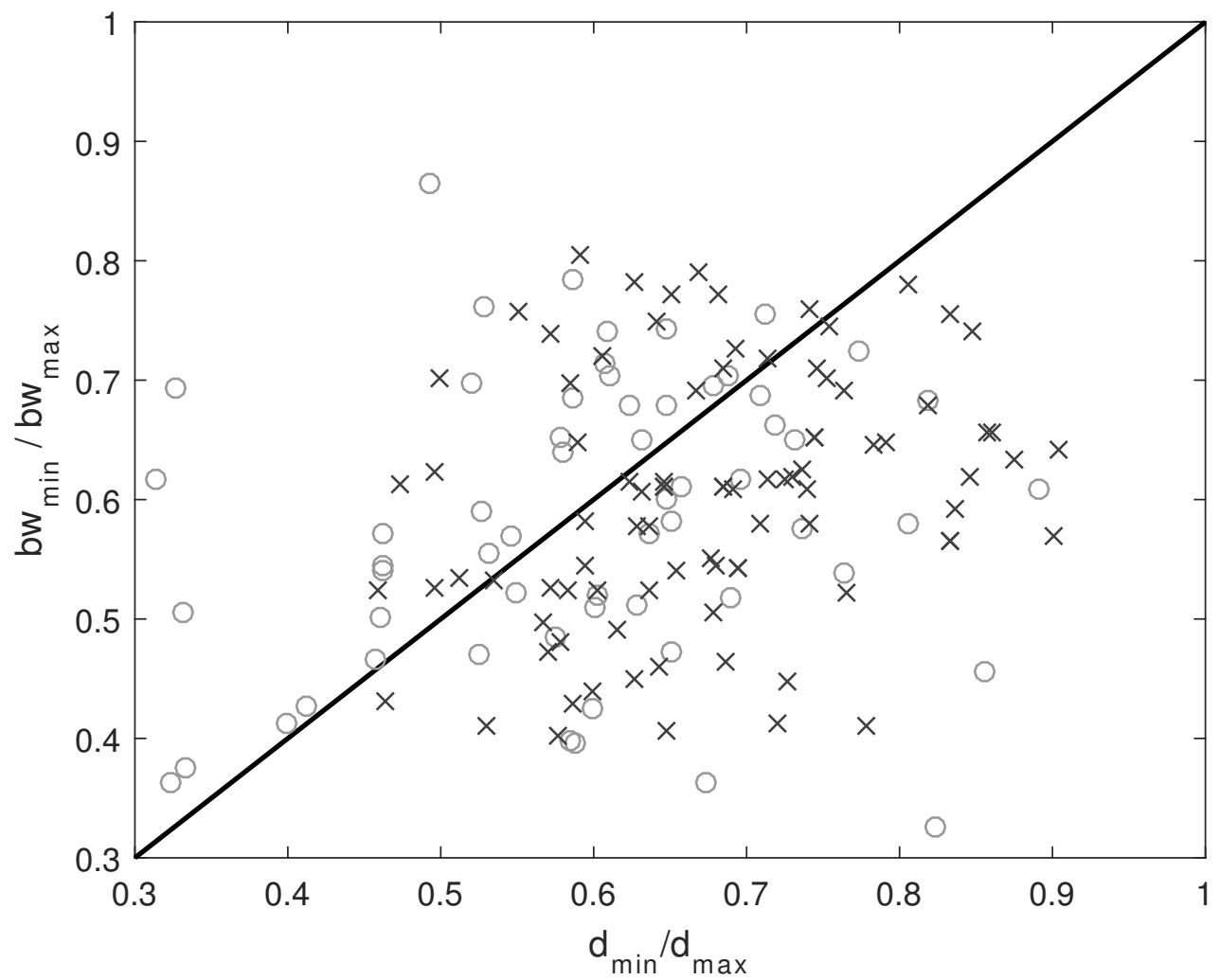

Figure 3.17: The eccentricity of the geometry is compared to the eccentricity of the beampattern. The black line in the middle represents the expected behaviour for ellipticaltransducers. The grey o's represent noseleaves while the black x's are pinnae. Each of the points on the graphs is the average eccentricity of the beamwidths for the aperture over its 10 measured frequencies. 


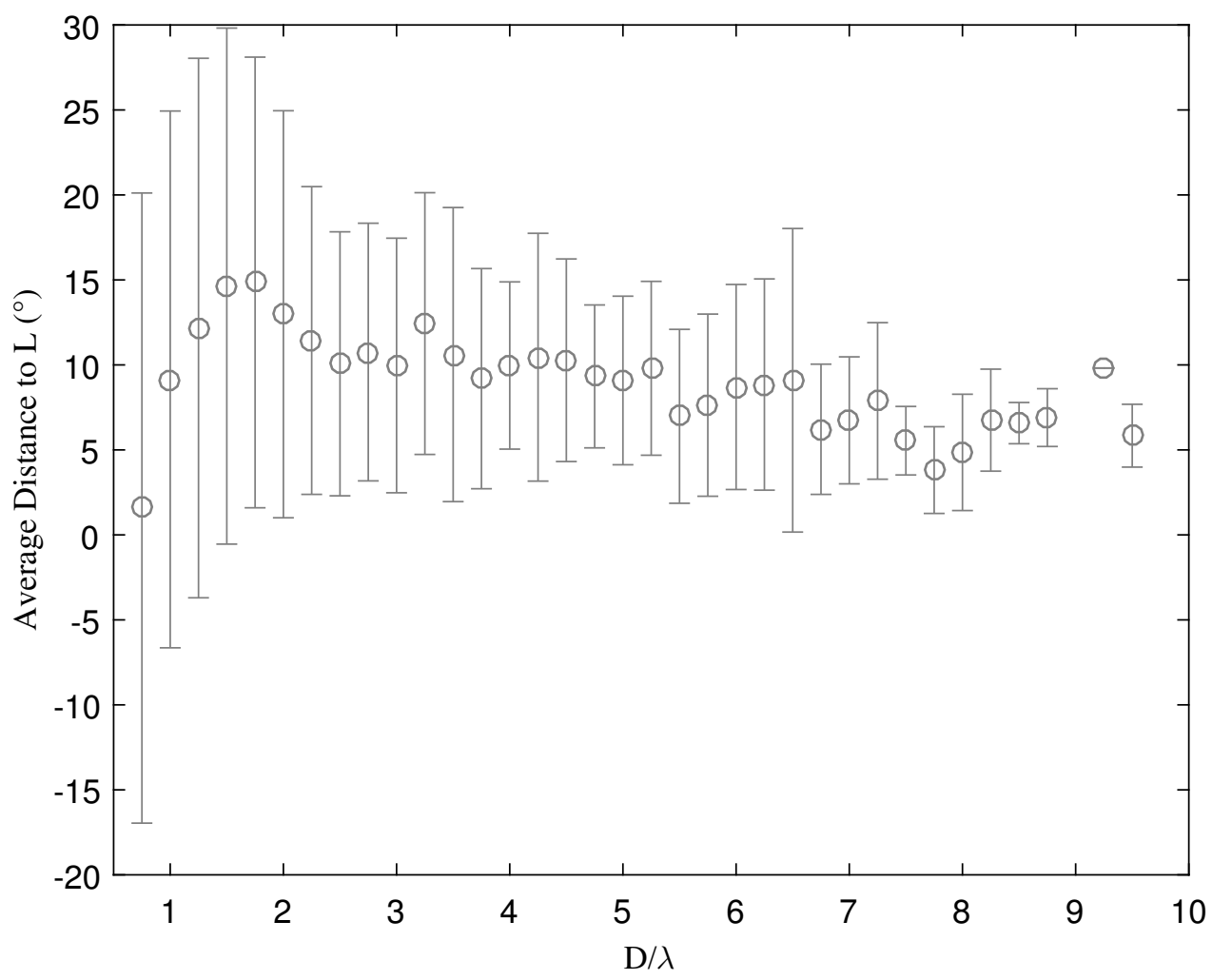

Figure 3.18: Distance between the beamwidths of the biosonar samples and the respective elliptical-transducer limit as a function of the diameter to wavelength ratio $\left(\frac{D}{\lambda}\right)$. The beamwidth data has been arranged in bins of width $0.25 \frac{D}{\lambda}$. The averages for all data points in each bin are indicated by open circles; the error bars mark the standard deviations. 


\section{Chapter 4}

\section{Discussion}

\subsection{Importance of Beamwidth in Biosonar}

The results obtained can be seen as indications that beamwidth may play very different roles in bat biosonar and technical sonar. With few exceptions, the biological and technical sonars included in the sample operated in very different diameter over wavelength regimes with technical sonars operating at much larger size to wavelength ratios than the bat biosonars. Only 4 out of the 23 technical sonars included in this study overlapped with the upper half of the range covered by the biosonar systems. Due to the basic inverse relationship between beamwidth and the ratio of aperture diameter and wavelength, the different operating regimes of biological and technical sonars were manifest in different beamwidths ranges: whereas the half-power bandwidths of technical sonars were typically much less than $10^{\circ}$, those of the biological sonars were typically much larger than $10^{\circ}$. This difference is to be expected based on the different sizes of biological and engineered sonar relative to the employed wavelength.

Indeed, the smallest beamwidths found in bats were predicted fairly well by the planar elliptical-transducer model. Small deviations towards smaller values, i.e., below the reference curve of the elliptical could be due to measurement errors, e.g., in determining the equivalent transducer diameters or in handling local shape features of the beampattern such as sidelobes. They could also be due to inadequacies the elliptical-transducer model may have in describing three-dimensional structures. In either case, it is noteworthy that the scatter of the data points above the reference curve was found to be much larger than that below the curve. If the scatter below the curve can be taken as a estimate for the effect 
of the various error sources associated with the current approach, it can be concluded that the much larger deviations in the biosonar data towards larger beamwidth were - to a large extent - not due to measurement error.

For the technical sonar systems the scatter away from the limit curve was found to be very small. This can be taken as an indication that beamwidth is seen as a critical system characteristic by sonar engineers who will increase the size of their devices until the beamwidth requirement of the respective application is met (or some other limit is reached). For the biological sonars, the large upward scatter can be seen as evidence that factors other than the need to minimize beamwidth have been important to the evolutionary beampattern design. Hence, bat species with a similar aperture-diameter-to-wavelength ratio can have very different beamwidths. It could be the case that the different species benefit from different beamwidths due to their specific sensory ecology or that the beamwidths are more of a byproduct of other beampattern characteristics and do not have a substantial impact on the animals' biosonar sensing capabilities by themselves.

The comparison between the bat biosonar structures and random aluminum baffles supports this hypothesis as it shows that biological samples have scatter away from the limit curve that is greater (or equal in places) than what would happen if the baffles were random and not designed for a specific beamwidth at all. Hence, it could be argued the beampatterns of the bats have been under the influence of evolutionary forces that were not aimed at beamwidth. An alternative explanation could be that the biological beamwidths are entirely the result of a random process (like the aluminum baffles), but that the randomness in the biological baffles produced a greater scatter, e.g., because the design space was wider than that of the crumpled aluminum cones.

A final argument against an evolutionary drive towards small beamwidth in bat biosonar comes from the comparison of bat families believed to have some of the most sophisticated biosonar systems in nature (hipposiderids and rhinolophids) and a family with little biosonar use (Pteropodidae). Since no difference was obvious in the beamwidths between those two groups, the results do not support the notion that narrow beamwidth is an adaptation for use of biosonar.

\subsection{Differences in Emission and Reception}

An interesting finding that could be interpreted in the direction of functional specializations in sonar beamwidth is beamwidth difference between emission (noseleaves) and reception (pinnae) beams, where noseleaves were found to be smaller relative to the em- 
ployed wavelengths and hence produced larger beamwidths. This systematic difference could be either the results of a functional specialization where biosonar systems benefit from operating with wider emission beams than what they use for reception. Alternatively, it could be a byproduct of other factors that push for noseleaves that are smaller than their respective ears. Examples of such factors could be feeding behaviors where, for example, the bat may have to inserts its snout into a flower for feeding on nectar. It could also be that a large noseleaf could interfere with forward vision in species where the eyes contain important sensory information. While bat species with large noseleaves do exist, e.g., the sword-nosed bats in the genus Lonchorhina, they seem to be few and far between [39]. On the functional side, detection of targets would benefit from emission and reception beams that act as "matched filters", i.e., have identical shapes so that the weighting imposed by the reception beam matches that of the emission. However, for other sensory tasks and signal-to-noise ratios that are not at a critical limit, this may not be the best solution. It may be noteworthy in this context that many bat species, such as the rhinolophids and hipposiderids that were prominently represented in the current sample, have very mobile pinnae that can be rotated over large angles, whereas the noseleaves can deform but are not subject to such large angular re-orientations [26]. In such a scenario, the wide emission beams could create an opportunity for conducting dynamic scans on the reception side where a larger volume is being swept over time. 


\section{Chapter 5}

\section{Conclusion}

\subsection{Summary}

Biosonar has typically been studied by itself and in relation to the environment of the user, typically bats. However, by creating a method for converting biosonar apertures into planar diameters, biosonar can be compared to engineered sonar in terms of commonly used metrics, particularly beamwidth. Beamwidth is commonly used by engineered sonar as a measure of the sonar's resolution and performance and can be calculated for the biosonar apertures using numerically generated beampatterns calculated from apertures derived from $\mu \mathrm{CT}$ scans of the bats.

Given the biosonar beamwidths and geometries a comparison between biosonar and technical sonar could be performed which identified three key differences between them: biosonar tended to have beamwidths at least an order of magnitude larger, biosonar beamwidths tended to be further away from the reference limit of an equivalent planar array, and biosonar tended to be located at much lower values of Aperture Diameter $(D)$, or array-length, to wavelength $(\lambda)$ ratios. The first two of these points were further highlighted upon conducting an experiment measuring the beamwidth of randomly generated aluminum baffles that were located in the same regime of aperture diameter to wavelength as the biosonar where, on average, it was shown that the randomly generated baffles were closer to the reference beamwidth than the biosonar.

The biosonar additionally showed differences between the emission and reception elements. The emission elements (noseleaves) tend to have smaller ratios of $D$ to $\lambda$ which in turn causes noseleaves to have larger beamwidths on average. This finding can be cou- 
pled with the finding that as the biosonar apertures increase in values of $D$ to $\lambda$ they tend to converge to the reference beamwidth limit resulting in smaller beamwidths compared to the limit. However, only reception elements are located at the higher values of $D$ to $\lambda$ where this convergence becomes more prevalent, indicating that narrow beamwidths may not have been evolutionarily important for biosonar emission elements or that large beamwidths may be beneficial in biosonar emission.

An additional finding that supports beamwidth potentially not being an evolutionary concern of bats can be seen by comparing the performance of pinnae used by families of bats with sophisticated active sonar to a family of bats that do not use active sonar. Both families were found to operate at equivalent $D$ to $\lambda$ ratios and have comparable beamwidths and distance from the reference limit at those $D$ to $\lambda$ regimes. This lends further credence to the concept that beamwidth may not be an important evolutionary factor for bats, unlike the importance that sonar engineers have placed on developing sonar arrays with smaller beamwidths.

\subsection{Conclusion}

The data present here supports the notion that technical and biological sonars may have radically different operational principles. Whereas high-resolution angular imaging of the world is key to technical sonars, bat biosonar tends to operate with beamwidth that are at least one order of magnitude larger than those of typical engineered systems. Part of the performance gap in terms of beamwidth and resolution observed in this research can be explained by the larger sizes of the engineered sonar compared to the biosonar, but even with engineered sonar that operate at the same values of array-length, or aperture diameter, to wavelength there was a noticeable increase in average biosonar beamwidth.

Nevertheless, the evolutionary success of bats and the ability of many bat species to thrive in complex environments, such as dense vegetation, indicates that the animals must have found a way to obtain the relevant sensory information in ways that does not rely on a narrow beamwidth and the high angular resolution it conveys. These strategies should be of great interest to engineers, because reproducing the abilities of bats could support bringing reliable navigation in complex environments to small platforms. 


\subsection{Importance of Results}

Prior to this research it had been quantitatively shown that biosonar beamwidths were larger than engineered sonar beamwidths. However, there were very few calculated beamwidths for the biosonar to use for comparisons, and the beamwidths calculated were for a single species of bat. This research created a large database of biosonar apertures and their beam information, across many different families and species of bat. This database contains all of the information used in comparison within this research, as well as additional information on the biosonar beampatterns such as locations and values of local minima and local maxima in the beampatterns and width of the main lobe of the biosonar. By using this database of information alongside the methods created for determining an engineering analogue for the biosonar apertures, it was able to be shown that the biosonar apertures have significant scatter from the reference limit.

This scatter is important for future research into understanding how biosonar operates. While it previously could be said that biosonar beamwidths were larger than engineered sonar beamwidths, it can now be shown in a large sample size that having a narrow beamwidth does not appear to be important to biosonar. The importance of this finding is that it means that bats may not be using alternative methods to supplement their wide beamwidths, but instead that narrow beamwidths are irrelevant or even potentially harmful to the operation of the biosonar. These results show that the future of biosonar research may not be in finding out how to use biosonar operating differences, such as dynamics of the baffle, to generate narrower beamwidths but instead attempting to understand these effects on their own and in relation to an entirely different sensing paradigm than what is used in engineered sonars.

These results are compounded by the additional tests and comparisons performed. The noseleaf simulate test further corroborated the idea that narrow beamwidths may actually be harmful to the operation of the biosonar apertures, as simulates of the same size and with random internal geometries and imperfections were found to have less scatter than their biosonar equivalents.

The importance of having a large sample set of data could be seen by the analysis of the emission and reception elements. Prior to this research there was not any known, quantifiable differences between the beampatterns of the emission and reception elements. Finding that the emission elements had statistically larger beamwidths than their reception counterparts places another piece in the understanding of how the biosonar systems operate and helps further focus future research into how engineering analogue systems can be created to mimic their performance. 


\subsection{Achievements}

In this research, I have devised a methodology for equating biosonar apertures to twodimensional transducers and calculating the beamwidth of numerically generated biosonar beampatterns. With these methods, I was able to show that biosonar apertures follow the general trend for beamwidth, though many samples did not follow the limit closely. Additionally, I was able to compare the biosonar to technical sonar to show that the biosonar had a performance gap not present in the technical sonar. An experiment I performed also showed that the biosonar apertures did not on average perform as well as randomly generated aluminum baffles, further highlighting a potential performance gap.

I am first author on one journal paper manuscript that has been submitted to Bioinspiration \& Biomimetics and I have presented posters on this research at two Naval Engineering Education Consortium (NEEC) conferences. 


\section{Bibliography}

[1] B. Barshan and R. Kuc. A bat-like sonar system for obstacle localization. IEEE T. Syst. Man Cyb., 22(4):636-646, Jul 1992.

[2] W. S. Burdic. Underwater Acoustic Systems Analysis. Prentice Hill, Englewood Cliffs, NJ, 2nd edition, 1991.

[3] P. Caspers, A. Leonessa, and R. Müller. Eigenbeam analysis of the diversity in bat biosonar beampatterns. J. Acoust. Soc. Am., 135, 2014.

[4] P. B. Caspers. Analysis of Bat Biosonar Beampatterns: Biodiversity and Dynamics. $\mathrm{PhD}$ thesis, Virginia Polytechnic and State University, 2017.

[5] EdgeTech. 4200 Side Scan Sonar System.

[6] Robot Electronics. Air Ultrasonic Ceramic Transducers.

[7] L. A. Estroff and A. D. Hamilton. At the interface of organic and inorganic chemistry: Bioinspired synthesis of composite materials. Chem. Mater, 13(10):32273235, 2001.

[8] T. Fortmann, Y. Bar-Shalom, and M. Scheffe. Sonar tracking of multiple targets using joint probabilistic data association. IEEE J.Oceanic Eng., 8(3):173-184, Jul 1983.

[9] Y. Fu, P. Caspers, and R. Müller. A dynamic ultrasonic emitter inspired by horseshoe bat noseleaves. Bioinspir. Boimim., 11(3):036007, 2016.

[10] D. R. Griffin, F. A. Webster, and C. R. Michael. The echolocation of flying insects by bats. Anim. Behav., 8:141-154, 1960.

[11] R. Harrington. Sidelobe reduction by nonuniform element spacing. IRE Transactions on Antennas and Propagation, 9(2):187-192, March 1961. 
[12] K.-Z. Ivanić, Z. Tadić, and M. A. Omazić. Biomimicry - an overview. The Holistic Approach to Environment, 5:19-36, 2015.

[13] G. Jones and M.W. Holderied. Bat echolocation calls: adaptation and convergent evolution. P. Roy. Soc. B-Biol. Sci., 274, 2007.

[14] J. Jonsson, E. Edqvist, H. Kratz, M. Almqvist, and G. Thornell. Simulation, manufacturing, and evaluation of a sonar for a miniaturized submersible explorer - correspondence. IEEE Xplore, 57, 2010.

[15] E. K.V. Kalko and H.-U. Schnitzler. The echolocation and hunting behavior of daubenton's bat, myotis daubentoni. Behav. Ecol. Sociobiol., 24:225-238, 1989.

[16] S. Kim, C. Laschi, and B. Trimmer. Soft robotics: a bioinspired evolution in robotics. Trends Biotechnol., 31(5):287 - 294, 2013.

[17] Kongsberg. Dual Frequency Side Scan Sonar.

[18] Kongsberg. High Resolution and Domed Sonar Heads.

[19] R. Kuc and B. Barshan. Bat-like sonar for guiding mobile robots. IEEE Contr.Sys., 12(4):4-12, Aug 1992.

[20] L. E. Llewellyn and S. J. Bainbridge. Getting up close and personal: The need to immerse autonomous vehicles in coral reefs. In OCEANS 2015 - MTS/IEEE Washington, pages 1-9, Oct 2015.

[21] MaxBotix. High Performance Sonar Range Finder.

[22] M. E. McConney, K. D. Anderson, L. L. Brott, R. R. Naik, and V. V Tsukruk. Bioinspired material approaches to sensing. Adv. Funct. Mater., 19(16):2527-2544, 2009.

[23] Sound Metrics. Specifications UL300.

[24] R. Müller. Numerical analysis of biosonar beamforming mechanisms and strategies in bats. J. Acoust. Soc. Am., 128(3):1414-1425, September 2010.

[25] R. Müller. Dynamics of biosonar systems in horseshoe bats. Eur. Phys. J. - Spec. Top., 224(17-18):3393-3406, December 2015.

[26] R. Müller, A.K. Gupta, H. Zhu, M. Pannala, U.S. Gillani, Y. Fu, P. Caspers, and J.R. Buck. Dynamic substrate for the encoding sensory information in bat biosonar. Phys. Rev. Lett., 118(15):158102, April 2017. 
[27] M. F. A. B. M. Murad, M. I. B. Samah, Z. H. Ismail, and K. Sammut. Surveillance of coral reef development using an autonomous underwater vehicle. In 2016 IEEE/OES Autonomous Underwater Vehicles (AUV), pages 14-19, Nov 2016.

[28] R. Müller and R. Kuc. Biosonar-inspired technology: goals, challenges and insights. Bioinspir. Biomim., 2(4):S146, 2007.

[29] G. Neuweiler. The Biology of Bats. Oxford University Press, New York, NY, 2000.

[30] H. Nguyen, J. Jonsson, E. Edqvist, and J. Sundqvist. Heavily miniaturized submerisible - a terrestrial kickoff. 2008.

[31] V. H. Pascual, T. F. Otero, and J. Schumacher. Biomimetic reactions in conducting polymers for artificial muscles: sensing working conditions. volume 10162, pages $10162-10162-8,2017$.

[32] D. J. Peters. A bayesian method for localization by multistatic active sonar. IEEE J Oceanic Eng, 42:135-142, 2016.

[33] R2Sonic. Sonic 2024/2022 Broadband Multibeam Echsounders.

[34] Reson. SeaBat 7125 Operator's Manual.

[35] Reson. SeaBat 7130 Operator's Manual.

[36] A. S. Rudolph, S. G. Wax, and L. Christodoulou. Bio-inspired dynamic robots, 2003.

[37] S. Schmidt, S. Hanke, and J. Pillat. The role of echolocation in the hunting of terrestrial prey - new evidence for an underestimated strategy in the gleaning bat, megaderma lyra. J. Comp. Physiol., 186:975-988, 2000.

[38] H U Schnitzler, E Kalko, L Miller, and A Surlykke. The echolocation and hunting behavior of the bat,pipistrellus kuhli. J. Comp. Physiol., 161:267-274, 1987.

[39] N. B. Simmons. Mammal Species of the World: A Taxonomic and Geographic Reference. Johns Hopkins University Press, Baltimore, 3rd edition, 2005.

[40] Sonardyne Solstice. Search and Classify Side Scan Sonar.

[41] Far Sounder. FarSounder-1000 Navigation Sonar.

[42] D. D. Sternlicht, J. E. Fernandez, J. L. Prater, J. N. Weaver, J. C. Isaacs, T. C. Montgomery, C. M. Loeffler, and M. Purcell. Advanced sonar technologies for high clearance rate mine countermeasures. 2016. 
[43] B. Su, Y. Tian, and L. Jiang. Bioinspired interfaces with superwettability: From materials to chemistry. J. Am. Chem. Soc., 138(6):1727-1748, 2016. PMID: 26652501.

[44] J. Sutlive, B. Todd, E. Sachdeva, B. Nguyen, A. Yu, A. Farley, and R. Müller. Biomimetic sonar for encoding target class at small scales. NEEC Annual Meeting 2017, April 2017.

[45] Teledyne. M900 Series.

[46] Teledyne. P450 Series.

[47] Teledyne. P900 Series Deepwater 2D Imaging Sonar.

[48] J. E. Thorner. Approaches to sonar beamforming. In IEEE Technical Conference on Southern Tier, pages 69-78, Apr 1990.

[49] R. J. Urick. Principles of Underwater Sound. McGraw-Hill Book Company, New York, NY, 3rd edition, 1983.

[50] Julian F.V Vincent, Olga A Bogatyreva, Nikolaj R Bogatyrev, Adrian Bowyer, and Anja-Karina Pahl. Biomimetics: its practice and theory. Journal of The Royal Society Interface, 3(9):471-482, 2006.

[51] Wassp. WMB-3230/WMB-5230/WMB-3250 Specifications.

[52] K.-K. Yan and Y. Lu. Sidelobe reduction in array-pattern synthesis using genetic algorithm. IEEE Transactions on Antennas and Propagation, 45(7):1117-1122, Jul 1997.

[53] A. Zhao, L. Ma, X. Ma, and J. Hui. An improved azimuth angle estimation method with a single acoustic vector sensor based on an active sonar detection system. Sensors, 17, 2017.

[54] Q. Zhuang and R. Müller. Noseleaf furrows in a horseshoe bat act as resonance cavities shaping the biosonar beam. Phys. Rev. Lett., 97(21):218701-4, November 2006. 


\section{Appendices}

\section{Appendix A: Code}

\section{Appendix A.1: Geometric Processing Code}

\%Main processing program used for turning edge filtered point clouds into $\% 2 \mathrm{D}$ geometry and calls subfunction for processing beampatterns to find \%beamwidths

\% A cell of strs (Referred to as str.mat) is loaded with each str $\%$ representing the file name associated with a point cloud and beampattern.

$\%$ Information for that aperture is loaded from the respective file

$\%$ location for processing.

$\%$ At the end of the program all of the information is loaded into and

$\%$ updated into a structure, containing the information generated on the

$\%$ aperture geometry and the beamwidths and beampatterns at the 10

$\%$ frequencies.

clear

$\mathrm{clc}$

close all

\%Load a cell of strings, str, corresponding to the aperture file names load ('str.mat', 'str')

\%Reassign the variable str to $1 \mathrm{str}$, as str was used in other places in the \%program

$1 \mathrm{str}=\mathrm{str}$

\%Load the current structure so that additional points can be added into \%the structure rather than made seperate

load vstruct.mat

for $i=1$ : length ( 1 str $)$ 
close all \%close all figure display windows for the previous run strelstr $\{i\} \%$ Udate str to represent the current file being process and \%display onscreen string for loading edge-filtered and edge-selected point \%cloud, changedirectory to match location string=strcat ('H: \Point Clouds \', str, 'PLY2.ply'); ptCloud=pcread ( string);

$\%$ String for loading beampattern, change directory to match location string $2=\operatorname{strcat}\left({ }^{\prime} \mathrm{H}: \backslash\right.$ allbeams $\backslash$ ', str ,' . mat' $)$;

load ( string 2 );

\%Reloact structure containing all data - Unsure why this was necessary but \%sometimes structure would not properly update with this in loop

load vstruct. mat

\% Extract locations from the point cloud and put into cartesian compoenents Loc $=$ ptCloud . Location ;

$\mathrm{x}=\operatorname{Loc}(:, 1)$;

$\mathrm{y}=\operatorname{Loc}(:, 2)$;

$\mathrm{z}=\operatorname{Loc}(:, 3)$;

\%Find midpoints between the maximum and minimum $\mathrm{x}, \mathrm{y}$, and $\mathrm{z}$ coordinates $\operatorname{midV}=[((\max (\mathrm{x})+\min (\mathrm{x})) / 2), \quad((\max (\mathrm{y})+\min (\mathrm{y})) / 2), \quad((\max (\mathrm{z})+\min (\mathrm{z})) / 2)]$; \%Perform an affine fit (root mean square) to find the plane of best fit $[\mathrm{n}, \mathrm{V}, \mathrm{p}]=\operatorname{affine}_{-} \mathrm{fit}_{(\mathrm{Loc})}$;

mean $\mathrm{V}=\mathrm{p}$;

$\% \%$ 3D surf of point cloud

$\%$ \#\#\# Uncomment to show a 3D surf of the pointcloud

$\%$

$\% \mathrm{~d}=-\mathrm{p} * \mathrm{n}$

$\% \quad[\mathrm{xx}, \mathrm{yy}]=\mathrm{ndgrid}(\min (\mathrm{x}):((\max (\mathrm{x})-\min (\mathrm{x})) / 10):(\max (\mathrm{x})), \min (\mathrm{y}):(\ldots$

$\%(\max (\mathrm{y})-\min (\mathrm{y})) / 10): \max (\mathrm{y}))$;

$\% \quad[\mathrm{xx}, \mathrm{yy}]=\mathrm{ndgrid}((\min (\mathrm{x})-\operatorname{mean}(\mathrm{x})):(\operatorname{mean}(\mathrm{x}) / 10):(\max (\mathrm{x})+\operatorname{mean}(\mathrm{x})), \ldots$

$\%(\min (\mathrm{y})-\operatorname{mean}(\mathrm{y})):(\operatorname{mean}(\mathrm{y}) / 10):(\max (\mathrm{y})+\operatorname{mean}(\mathrm{y})))$;

$\% \quad \mathrm{zp}=(-\mathrm{n}(1) * \mathrm{xx}-\mathrm{n}(2) * \mathrm{yy}-\mathrm{d}) / \mathrm{n}(3) ;$

$\%$ figure

$\% \quad \operatorname{surf}(\mathrm{xx}, \mathrm{yy}, \mathrm{zp})$

$\%$ hold on

$\% \quad$ scatter $3(\mathrm{x}, \mathrm{y}, \mathrm{z})$

$\%$ axis equal

$\%$ New Plane Generation

\%Generate a plane of best fit from the values foud using the affine fit 
syms xvar yvar zvar \%Sym variables used to define plane $\mathrm{P}=[\mathrm{xvar}, \mathrm{yvar}, \mathrm{zvar}]$; \%Create array of sym variables for plane \%perform dot product of normals from RMS plane of best fit and a \%point on the plane planefunction $=\operatorname{dot}(n, P-p)$;

zplane $=$ solve (planefunction, zvar); \%Create the plane of best fit $\%$ Mesh plane and plot to see

fmesh (zplane, $[\min (\operatorname{Loc}(:, 1)) \max (\operatorname{Loc}(:, 1)) \min (\operatorname{Loc}(:, 2)) \max (\operatorname{Loc}(:, 2))])$ axis equal

hold on

\% Plot original catersian coordinates on plaen for comparison scatter $3(\operatorname{Loc}(:, 1), \operatorname{Loc}(:, 2), \operatorname{Loc}(:, 3))$

$\%$ Project onto plane

\%Perform projection of 3D points onto 2D plane of best fit. proj=Loc $*$ null $\left(n^{\prime}\right)$; \%create $x, y$ array of projected points

figure

scatter $(\operatorname{proj}(:, 1), \operatorname{proj}(:, 2)) \%$ plot projected points to compare shape to 3D ax is equal

hold on

\%Find mean of projected $x$ points - Depreciated in Use meanprojx $=(\operatorname{mean}(\operatorname{proj}(:, 1)))$;

\%Find mean of projected y points - Depreciated in Use meanprojy $=($ mean $(\operatorname{proj}(:, 2)))$;

scatter (meanprojx, meanprojy, 'r') \%Scatter the mean point onto plot $\%$ Find the middle of proj $x$ points $\operatorname{midprojx}=(\max (\operatorname{proj}(:, 1))+\min (\operatorname{proj}(:, 1))) / 2$; $\operatorname{midprojy}=(\max (\operatorname{proj}(:, 2))+\min (\operatorname{proj}(:, 2))) / 2 ; \%$ Find the middle of y points scatter (midprojx, midprojy, 'g') \%Scatter mid point onto plot axisv=axis; \%Save current axis for later use

$\%$ Calculate Distance Moved

$\%$ This set of code finds how far the points had to move in order to be on $\%$ the plane of best fit

$\mathrm{nr}=[1 ; 0 ; 0]$;

rrotvec $=\operatorname{vrrotvec}\left(\mathrm{n}^{\prime}, \mathrm{nr}^{\prime}\right)$;

mrotvec $=$ vrrotvec $2 \mathrm{mat}(\operatorname{rrotvec})$;

Locr $=$ Loc $*$ mrotvec '; 
Locrm $=$ mean $($ Locr $)$;

Locrmc=bsxfun (@minus, Locr, Locrm );

$[$ nra, Vra,pra $]=$ affine fit $_{(\text {Locrmc })}$;

projr $=$ Locrmc $*$ null (nra');

distmoved=Locrmc $(:, 1)$; \%array of how far each point moved

dmmean=mean (distmoved); \%mean distance moved by all points

dmstd=std(distmoved); \%standard deviation of distanced moved by all points

$\%$ Find Principle Components

$\%$ Find Principle compoennts of the 2D projection. This has since

$\%$ depreciated from use for processing as it was decided that rather than

$\%$ use the principle components of the shapes, the minimum and maximum

$\%$ through the centroid (found later in code) would be used for comparison

$\%$ but has been kept in code for comparison purposes

coeff=pca $($ proj $)$; \%Find PCA coefficients

$\mathrm{x} 1=\operatorname{coeff}(:, 1)$;

$\mathrm{x} 2=\operatorname{coeff}(:, 2)$;

$\%$ Get slope of the first component

slope $1=(x 1(2)-$ meanprojy $) /(x 1(1)-$ meanprojx $)$;

yint $1=$ meanprojy-slope $1 *$ meanprojx $\%$ Get $y$ intercept of first component

slope $2=(x 2(2)-$ meanprojy $) /(x 2(1)-$ meanprojx $) ; \%$ Get slope of the compoenent

yint $2=$ meanprojy - slope $2 *$ meanprojx; $\%$ Get y intercept of second component

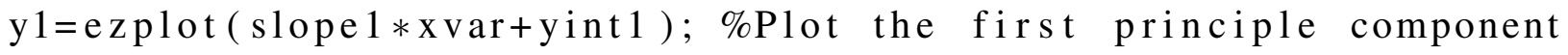

$\mathrm{y} 2=\mathrm{ezplot}(\mathrm{slope} 2 * \mathrm{xvar}+\mathrm{yint} 2) ; \% \mathrm{Plot}$ the second principle component

axis(axisv) \%Reset axis to earlier axis for easier viewing

$\%$ Find Length of intersection

\%Find the minimum and maximum distance from the principle components: This \%part of code is depreciated in use as the minimum and maximum through the \%centroid, found later in the code, are instead used for comparison but the \%code has been left as a point of comparison to the other values $\%$ create an $x$ linelength that spans the entire shape xilength $=$ linspace $(\min (\operatorname{proj}(:, 1)), \max (\operatorname{proj}(:, 1)) * 1.5)$;

yi $1=$ slope $1 * x$ ilength $+y i n t 1$; \% Create a line array corresponding to PC1 
yi $2=\operatorname{slope} 2 * x$ ilength $+y$ int $2 ; \%$ Create a line array corresponding to PC2

$\%$ Find PC1 length inside of shape polygon

[xint 1, yint 1$]=$ polyxpoly $(x i l e n g t h, y i 1, \operatorname{proj}(:, 1), \operatorname{proj}(:, 2))$;

$\%$ Find PC2 length inside of shape polygon

[xint 2, yint2]= polyxpoly $(x i l e n g t h, y i 2, \operatorname{proj}(:, 1), \operatorname{proj}(:, 2))$;

xint 1 max $=\max (x i n t 1)$; \%Find the largest $X$ value of PC1 inside the polygon xint $1 \mathrm{~min}=\min (\mathrm{xint} 1)$; \%Find the smallest $X$ value of PC1 inside the polygon yint 1 max $=\max (y$ int 1 ); \%Find the largest Y value of PC1 inside the polygon yint $1 \mathrm{~min}=\min (\mathrm{yint} 1$ ); \%Find the smallest Y value of PC1 inside the polygon $\%$ Find length of interior points $\mathrm{d} 1=\operatorname{sqrt}\left((\mathrm{xint} 1 \max -\mathrm{xint} 1 \min )^{\wedge} 2+(\operatorname{yint} 1 \max -\mathrm{yint} 1 \mathrm{~min})^{\wedge} 2\right)$; $\mathrm{xint} 1 \mathrm{mat}=[\mathrm{xint} 1 \mathrm{max} ; \mathrm{xint} 1 \mathrm{~min}] ; \%$ Create $\mathrm{X}$ vector to Plot yint $1 \mathrm{mat}=[$ yint $1 \mathrm{max}$; yint $1 \mathrm{~min}]$; \%Create Y Vector to plot $\%$ Plot intersection of 1 st principle component plot (xint1mat, yint1mat,'--, ', linewidth', 2 )

xint $2 \max =\max (x i n t 2)$; \%Find the largest $X$ value of PC2 inside the polygon xint 2 min=min( $x$ int 2 ); \%Find the smallest $X$ value of PC2 inside the polygon yint 2 max $=\max (y$ int2); \%Find the largest Y value of PC2 inside the polygon yint $2 \mathrm{~min}=\min (\mathrm{yint} 2$ ); \%Find the smallest Y value of PC2 inside the polygon $\%$ Find length of interior points $\mathrm{d} 2=\operatorname{sqrt}\left((x \text { int } 2 \max -\mathrm{xint} 2 \min )^{\wedge} 2+(\operatorname{yint} 2 \max -\mathrm{yint} 2 \min )^{\wedge} 2\right)$ $\mathrm{x}$ int $2 \mathrm{mat}=[\mathrm{xint} 2 \mathrm{max}$; $\mathrm{xint} 2 \mathrm{~min}] ; \%$ Create $\mathrm{X}$ vector to Plo yint 2 mat $=[$ yint 2 min; yint $2 \max ]$; \%Create Y Vector to plot $\%$ Plot intersection of 2 nd principle component plot (xint2mat, yint2mat, '--','linewidth',2)

title ('Projected Convex Hull on Plane of Best Fit') xlabel('Distance in $\mathrm{x}$-direction (m)') ylabel('Distance in $y$-direction (m)')

legend('Point Cloud',' Mean Point', 'Center point', 'Prime Component 1',... 'Prime Component 2', 'Overlap Vector 1', 'Overlap Vector 2')

$\% \%$ Convex Hull method

$\%$ Use of convex hull method to find the centroid of the 2D baffle shape \%Convert the projection $x$ and $y$ values to double format 
projd=double ( proj);

$x d=\operatorname{projd}(:, 1)$;

$y d=\operatorname{projd}(:, 2)$;

chull=convhull(xd,yd); \%Create convex hull from projection

$x c h u l l=x d(c h u l l) ; \%$ Create $x$ vector containing only points in the convex hull

ychull=yd(chull); \% Create y vector containing only points in the convex hull

chullp $=[\mathrm{xchull}$, ychull $]$; \%Create 2D convex hull array

xchullmean=mean ( $x$ chull); \%Find mean of convex hull in $x$

ychullmean=mean (ychull); \%Find mean of convex hull in $y$

coeff=pca(chullp); \%Create PCA coefficients of the convex hull

figure

\%Create a plot of the convex hull and the mean points

scatter (xd ( chull), yd ( chull))

axis equal

axisv=axis ;

hold on

scatter (xchullmean, ychullmean, 'r')

\% The following section generates slops and plots from the PCA components

$\%$ for the convex hull. The method is the same as earlier PCA line

$\%$ generation (Code line starting 109 for line by line comments)

$\mathrm{x} 1=\operatorname{coeff}(:, 1)$;

$\mathrm{x} 2=\operatorname{coeff}(:, 2)$;

slope $1=(x 1(2)-$ ychullmean $) /(x 1(1)-x c h u l l m e a n)$;

yint $1=$ ychullmean - slope $1 * x$ chullmean ;

slope $2=(x 2(2)-y c h u l l m e a n) /(x 2(1)-x$ chullmean $)$;

yint $2=$ ychullmean - slope $2 * x$ chullmean ;

$\mathrm{y} 1=\mathrm{ezplot}($ slope $1 * \mathrm{xvar}+\mathrm{yint} 1)$;

$\mathrm{y} 2=\mathrm{ezplot}(\operatorname{slope} 2 * \mathrm{xvar}+\mathrm{yint} 2)$;

axis ( axisv)

title ('Projected Convex Hull on Plane of Best Fit')

xlabel('Distance in $x$-direction (m)')

ylabel('Distance in y-direction (m)')

legend('Point Cloud', 'Mean Point', 'Prime Component 1', ...

'Prime Component 2') 
$\%$ Principle Component Method 3

\% Use principle components to determine and minimum and maximum distance \% While this method is calculated, the method in the later section,

$\%$ Min and Max distances, was used in final results. However, in the first $\%$ part of the section the centroid of the geometric shape is found

\% Create an array of points over the $x$ and $y$ length of the convex hull and $\%$ then find which points fall within the polygon. This points can be used $\%$ as a representation of the area of the polygon to find the centroid $\mathrm{xpchull=linspace}(\min (\mathrm{xchull}), \max (\mathrm{xchull}))$; ypchull=linspace $(\min ($ ychull $), \max ($ ychull $))$;

[xdchull, ydchull]=meshgrid (xpchull, ypchull);

inchull=inpolygon (xdchull, ydchull, xchull, ychull); \%Find grid points inside xdinchull=xdchull (inchull); \%X grid points that are in ydinchull=ydchull (inchull); \%Y grid points that are in chullpdin=[xdinchull, ydinchull]; \%array of points that are in

xdinchullmean=mean ( $x$ dinchull); \%Mean of interior X Points - X centroid ydinchullmean=mean (ydinchull); \%mean of interior y points - y centroid coeff=pca(chullpdin); \%Create PCs of the interior grid

figure

plot (xchull, ychull, 'linewidth, ,2);

scatter (xdinchull, ydinchull, 'marker', '. ');

axis equal

hold on

scatter (xdinchullmean, ydinchullmean, 'r', 'linewidth',4)

\%Perform PC analysis to find minimum and maximum of the grid with respect $\%$ to the principle components. Min/Max values used were calculated in later $\%$ section. For Line-by-Line explanation, the same calculations are done \%with explanation at code line 126. These are equivilent calculations \%using a different set of variables 
$\mathrm{x} 1=\mathrm{coeff}(:, 1)$;

$\mathrm{x} 2=\mathrm{coeff}(:, 2)$;

slope $1=(x 1(2)-y d i n c h u l l m e a n) /(x 1(1)-x d i n c h u l l m e a n)$;

yint $1=y d$ inchullmean - slope $1 * x$ dinchullmean ;

yint 1 s a ve $=y$ int 1 ;

slope $2=(x 2(2)-y d i n c h u l l m e a n) /(x 2(1)-x$ dinchullmean $) ;$

yint $2=y d$ inchullmean - slope $2 * x$ dinchullmean ;

$\mathrm{y} 1=\mathrm{ezplot}($ slope $1 * \mathrm{xvar}+\mathrm{yint} 1)$;

$\mathrm{y} 2=\mathrm{ezplot}(\operatorname{slope} 2 * \mathrm{xvar}+\mathrm{yint} 2)$;

axis ( axisv)

title ('Projected Convex Hull on Plane of Best Fit')

xlabel('Distance in $\mathrm{x}$-direction (m)')

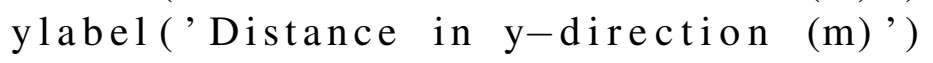

legend ('Evenly Distributed Point Cloud',' Mean Point, , ...

'Prime Component 1', 'Prime Component 2')

xilength $=1$ inspace $(\min (\operatorname{proj}(:, 1)), \max (\operatorname{proj}(:, 1)) * 1.5)$;

yi $1=\operatorname{slope} 1 *$ xilength + yint 1 ;

yi $2=$ slope $2 * x$ ilength + yint 2 ;

[xint 1, yint 1$]=$ polyxpoly $(x$ ilength $, y i 1, \operatorname{proj}(:, 1), \operatorname{proj}(:, 2))$;

$[$ xint 2, yint2 ] = polyxpoly $(x i l e n g t h, y i 2, \operatorname{proj}(:, 1), \operatorname{proj}(:, 2))$;

xint $1 \max =\max (x$ in 1$)$;

xint $1 \min =\min (x$ int 1$)$;

yint 1 max $=\max ($ yint 1$)$;

yint $1 \mathrm{~min}=\min (\mathrm{y}$ int 1$)$;

$\mathrm{d} 1=\operatorname{sqrt}\left((\mathrm{xint} 1 \max -\mathrm{xint} 1 \min )^{\wedge} 2+(\operatorname{yint} 1 \max -\mathrm{yint} 1 \min )^{\wedge} 2\right)$

xint $1 \mathrm{mat}=[\mathrm{xint} 1 \mathrm{max}$; $\mathrm{xint} 1 \mathrm{~min}]$;

yint $1 \mathrm{mat}=[\mathrm{yint} 1 \mathrm{max}$; yint $1 \mathrm{~min}]$;

plot (xint 1 mat, yint 1 mat ,' - - ', 'linewidth ',2 )

$\mathrm{xint} 2 \mathrm{max}=\max (\mathrm{x}$ int 2$)$;

$\mathrm{x}$ int $2 \min =\min (\mathrm{x}$ int 2$)$;

yint $2 \max =\max (\mathrm{y}$ int 2$)$;

yint $2 \min =\min ($ yint 2$)$;

$\mathrm{d} 2=\operatorname{sqrt}\left((\operatorname{xint} 2 \max -x \operatorname{int} 2 \min )^{\wedge} 2+(\operatorname{yint} 2 \max -y i n t 2 \min )^{\wedge} 2\right)$

$\mathrm{x}$ int $2 \mathrm{mat}=[\mathrm{xint} 2 \mathrm{max}$; $\mathrm{x}$ int $2 \mathrm{~min}]$;

yint $2 \mathrm{mat}=[\mathrm{yint} 2 \mathrm{~min} ; \mathrm{yint} 2 \mathrm{max}]$; 
plot (xint2mat, yint2mat, '--,', linewidth ',2)

$\%$ Find Rotational Information - Currently Depreciated

\%This section finds the amount of rotation for ponting the 3D mesh along

\%the $x$-axis

$\%\{$

$\mathrm{a}=[1 ; 0 ; 0]$;

$\mathrm{nrota}=\mathrm{n}-\mathrm{a}$;

nrota $=$ nrota $/$ norm $($ nrota $)$;

$\operatorname{deg} x=\operatorname{acosd}\left(\operatorname{nrota}(1) / \operatorname{sqrt}\left(\operatorname{nrota}(1)^{\wedge} 2+\operatorname{nrota}(2)^{\wedge} 2+\operatorname{nrota}(3)^{\wedge} 2\right)\right)-90 ;$

$\operatorname{deg} y=\operatorname{acosd}\left(\operatorname{nrota}(2) / \operatorname{sqrt}\left(\operatorname{nrota}(1)^{\wedge} 2+\operatorname{nrota}(2)^{\wedge} 2+\operatorname{nrota}(3)^{\wedge} 2\right)\right)$;

$\operatorname{deg} z=\operatorname{acosd}\left(\operatorname{nrota}(3) / \operatorname{sqrt}\left(\operatorname{nrota}(1)^{\wedge} 2+\operatorname{nrota}(2)^{\wedge} 2+\operatorname{nrota}(3)^{\wedge} 2\right)\right)$;

$\operatorname{deg} d=[\operatorname{deg} x, \operatorname{deg} y, \operatorname{deg} z]$;

$\operatorname{deg} d=\operatorname{round}(\operatorname{deg} d)$;

$\%\}$

$\%$ Max and Min Distances

\%This sections finds the minimum and maximum distances through the centroid xd; \% projected x values in double format

yd; \% projected y values in double format

xdinchullmean; $\% x$ value of mean by convex hull method

ydinchullmean; $\%$ y value of mean by convec hull method

$x d o=x d-x d i n c h u l l m e a n ; \% t r a n s l a t i o n$ of $x d$ mean onto origin

ydo=yd-ydinchullmean; \%translation of yd mean onto origin

\% Plot figure to make sure it still looks right, just translated

figure

hold on

plot ( xdo, ydo)

axis equal

axispl=axis ;

\%Find minimum and maximum values of the translated convex hull and set them $\%$ to be slightly further out to gaurantee overlap on all sides of the \%polygon

$x$ domax $=\max (x d o)$;

$x \operatorname{domin}=\min (\mathrm{xdo})$; 


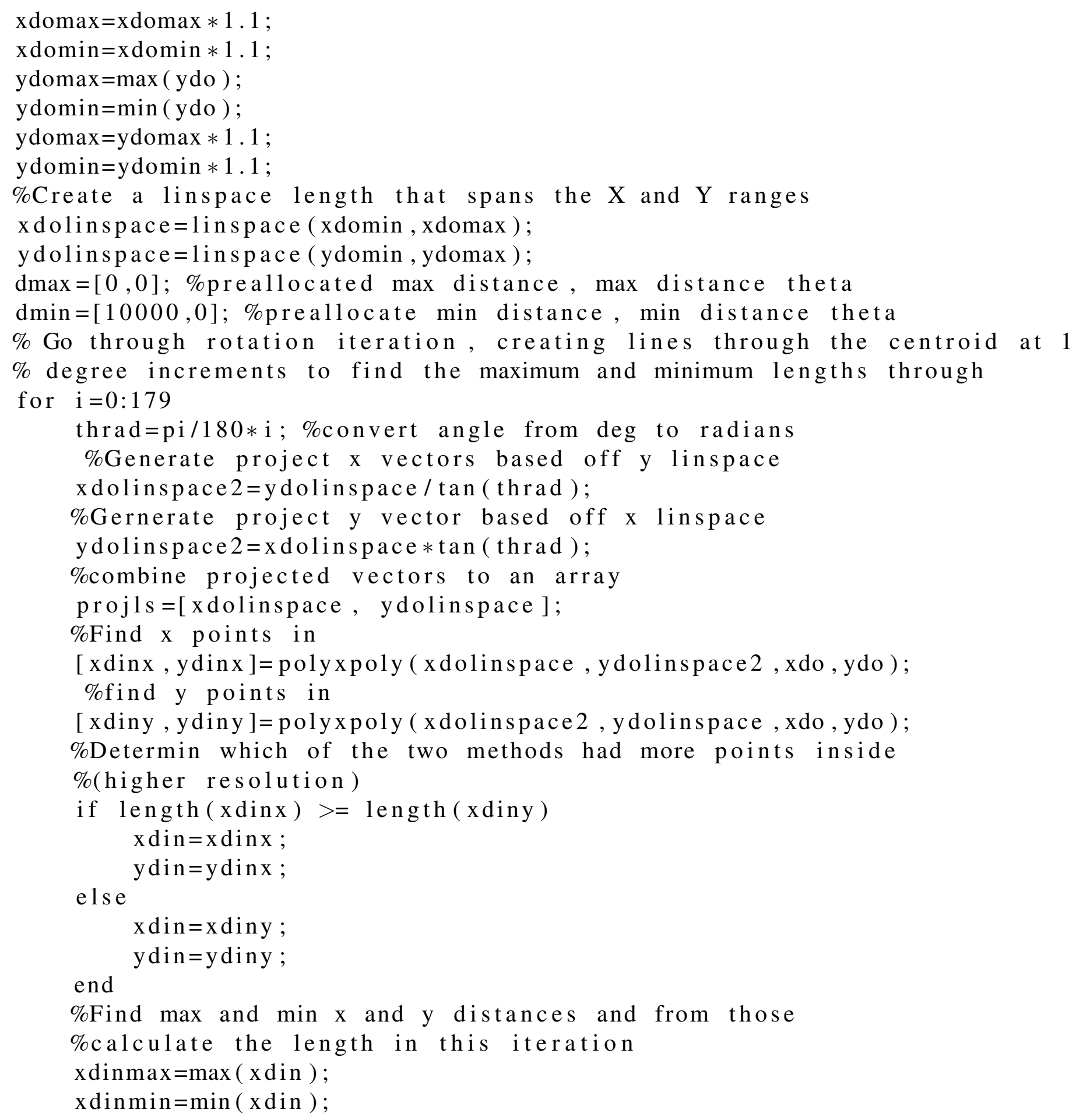




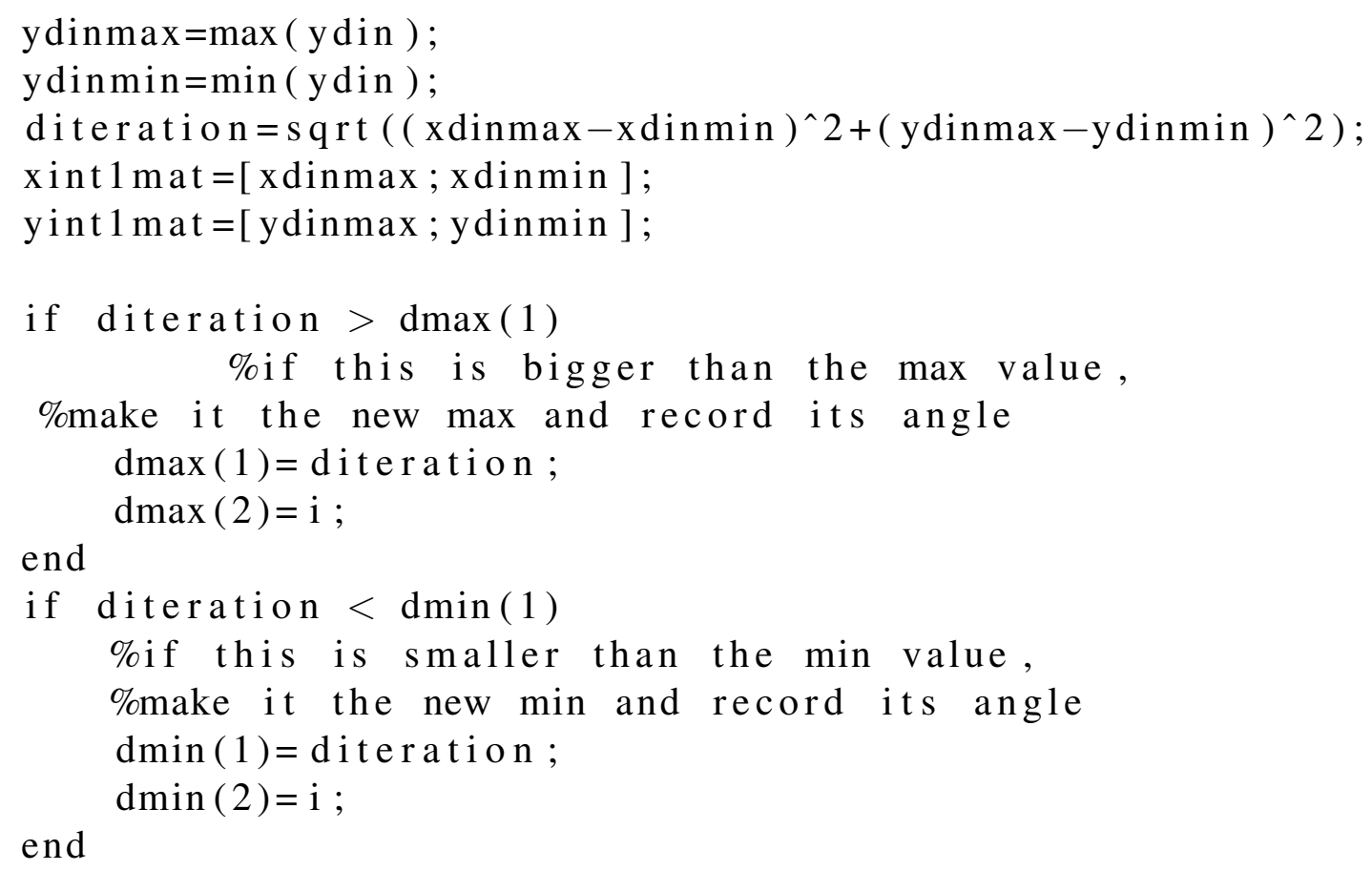

end

dmax; \%Display the final max value found

dmin; \%Display the final min value found

$\%$ Plot min and $\max$ vectors

d maxth=dmax $(2)$;

ydolinspace $=x d o l i n s p a c e * \tan (\operatorname{dmaxth} *$ pi $/ 180)$;

hold on

plot (xdolinspace, ydolinspace ,' -- ', 'linewidth ',2 )

d minth $=$ dmin $(2)$;

ydolinspace $=x d o l i n s p a c e * \tan (\operatorname{dminth} *$ pi $/ 180)$;

hold on

plot (xdolinspace, ydolinspace ,'--, ,' linewidth ',2 )

axis ( axispl);

$\% \%$ Input Geometric information in value structure

fieldstructname=str; \%Set the field name for saving as the file name 
\%Save the geometric information in the stucture valstruct. (fieldstructname). geom=struct ('dmax', dmax, 'dmin', dmin, ...

'distmoved', distmoved, 'dmmean', dmmean, 'dmstd', dmstd);

$\% \%$ Generate Beamwidth information from beampattern at frequencies

for $\mathrm{i}$ i $=1: 10$

[bwmax, bwmin, bwsmax, bwsmin, rdbmax, rdbmin, Fm, valmat]... $=$ process - pattern ( $\operatorname{string} 2$, i i $)$;

$\%$ Create Structure Information

freqfield=sprintf(' freq\%d', ii )

valstruct. (fieldstructname). (freqfield)=struct ('rdbmin', rdbmin, ' $\operatorname{rdbmax},, \ldots$ rdbmax, 'bwsmax', bwsmax, 'bwsmin', bwsmin, 'interpvalues ', valmat);

moviename $=$ strcat $($ str , ' freqnum, , num $2 \operatorname{str}($ ii $))$;

mvsavename $=$ strcat ('Figures /', moviename);

save (mvsavename, 'Fm');

$\%$ Save movie file seperately - Movie file is a 180 frame movie of the $\%$ progression of the $2 \mathrm{D}$ beamwidths that can't be saved in the structure

end

\%Save the structure for reloading next pass save('vstruct.mat', 'valstruct')

end

\section{Appendix A.2: Beampattern processing Code}

function [bwmax, bwmin, bwsmax, bwsmin, rdbmax, rdbmin, Fm, valmat]=.. process_pattern (mat_file_name, freqnum)

$\%$ This is a function for taking a beampattern file comprised of gain as a

$\%$ function of azimuth and elevation, and then finding the $-3 \mathrm{~dB}$ and $-6 \mathrm{~dB}$

$\%$ beamwidths, both including and excluding sidelobes

\%Verify the file we are looking for exists if ( exist ('mat_file_name', 'var'))

fprintf(' Must enter mat file name containing beam pattern data $\backslash$ ') ; 
return;

end

\%Load the beampattern file, including the variables of:

$\%$ G: Gain, K: Frequency, naz: Number of Azimuth angles, nel: Number of \%elevation angles

load (mat_file_name, 'G', 'K', 'naz', ' nel' ) ;

$\%$ Pull out the gain matrix for the frequency we are working with

$\mathrm{G} 1=\mathrm{G}(:,:$, freqnum $)$;

$\mathrm{x} 1=[-180: 1: 180]$; \%Create a set of $\mathrm{X}$ coordinates in degrees

y1 $=[-90: 1: 90]$; \% Create a set of y coordinates in degrees

$\%$ For each angle of azimuth and elevation create a corresponding $\mathrm{X}$ and $\mathrm{Y}$ $\%$ value

for $\mathrm{ii}=1: \mathrm{naz}$

$\mathrm{Y}(:, \mathrm{ii})=\mathrm{y} 1$;

end

for $\mathrm{ii}=1:$ nel

$\mathrm{X}(\mathrm{ii},:)=\mathrm{x} 1$;

end

$\%$ Plot for Mesh

$\%$ Uncomment this section to plot mesh to look for inconsistencies $\%\{$

figure ;

$\operatorname{mesh}(\mathrm{X}, \mathrm{Y}, \mathrm{G} 1)$;

$\%\}$

$\% \%$ Reshape the beampattern

\% This section reshapes the beampattern in order to convert it from $\%$ spherical coordinate to cartesian coordinates for easier work later

$X_{-}$reshaped $=$reshape $(X, 1, n a z *$ nel $)$;

$\mathrm{Y}_{-}$reshaped $=\operatorname{reshape}(\mathrm{Y}, 1, \mathrm{naz} * \mathrm{nel})$;

$\mathrm{G}_{-}$reshaped $=$reshape $(\mathrm{G} 1,1, \mathrm{naz} * \mathrm{nel})$;

$\mathrm{Az}=\mathrm{X}_{-}$reshaped $*$ pi/180;

De $=Y_{-}$reshaped $*$ pi $/ 180$;

$[\mathrm{CX}, \mathrm{CY}, \mathrm{CZ}]=\operatorname{sph} 2 \mathrm{cart}\left(\mathrm{Az}, \mathrm{De}, \mathrm{G}_{-}\right.$reshaped $) ;$ 
$\%$ Plot for Reshaped Beam Pattern

$\%$ Uncomment to view the reshaped beam pattern

$\%\{$

figure ;

hold on ;

plot3 (CX, CY, CZ);

axis $\left(\left[\begin{array}{llllll}-1 & 1 & -1 & 1 & -1 & 1\end{array}\right]\right.$,' equal');

$\%\}$

$\% \%$

$\%$ Find the Beam magnitude: This should be 1 , but may not be if there were \%problems with the simulation

beam_mag $=$ beam_max $(\mathrm{CX}, \mathrm{CY}, \mathrm{CZ})$;

$\%$ Find the location of the MRA

[ beam_max_val, beam_max_point] = $\max ($ beam_mag $)$;

\%Uncomment to see initial location in $x, y, z$ of MRA

CX(beam_max_point);

CY(beam_max_point);

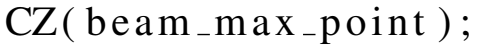

$\%-$ Do first rotation around $\mathrm{Z}$ axis to get MRA on $\mathrm{X}-\mathrm{Y}$ plane --

$\%$ theta $=\operatorname{atan}(C Y($ beam_max_point $) / C X($ beam_max_point $)) ;$

$\%$ theta $=-\operatorname{atan} 2\left(\mathrm{CY}\left(\right.\right.$ beam_max moint $\left._{-}\right), \mathrm{CX}\left(\right.$ beam_max moint $\left.\left._{-}\right)\right)$;

theta $=-\operatorname{atan} 2\left(\mathrm{CZ}\left(\right.\right.$ beam_max point $\left._{-}\right), \mathrm{CX}\left(\right.$ beam_max boint $\left.\left._{-}\right)\right)$;

$\%$ RZ = calculate RZ $_{(\text {theta })}$

$\mathrm{RY}=\mathrm{calculate}_{-} \mathrm{RY}($ theta $)$

$[\operatorname{dum}$ nrows $]=\operatorname{size}(\mathrm{CX})$;

rot_mat $=$ zeros(nrows, 3); \%Create a placeholder for the rotated matrix rot_mat $_{-}(:, 1)=\mathrm{CX}$;

rot_mat $(:, 2)=C Y$;

rot_mat $(:, 3)=\mathrm{CZ}$;

$\%$ rotated $=$ rot_mat $* \mathrm{RZ}$

rotated $=$ rot $_{-}$mat $* \mathrm{RY}$; 
$\%$-now find max again and rotate it arounc $\mathrm{Y}$ to the $\mathrm{Z}$ axis --

beam_mag = beam_max $($ rotated $(:, 1)$, rotated $(:, 2)$, rotated $(:, 3)) ; \%$ Find magnitudes $\%$ Find location and value of magnitudes

[ beam_max_val beam_max_point] = $\max ($ beam_mag $)$;

rotated (beam_max_point , 1), rotated (beam_max_point , 2 ), . .

rotated (beam_max_point, 3 ) \%displayer location of max magnitude

$\%$ phi $=\operatorname{atan}\left(\right.$ rotated $($ beam_max_point, 3$) /$ rotated $\left(\right.$ beam_max $\_$point, 1$\left.)\right) ;$

$\%$ phi $=-\operatorname{atan} 2\left(\right.$ rotated $(\text { beam_max } \text { point }, 3)_{-}$, rotated (beam_max_point, 1$\left.)\right)$;

$\%$ get rotation

phi $=\operatorname{atan} 2\left(\right.$ rotated $($ beam_max_point, 2$)$, rotated $\left(\right.$ beam_max $\_$point, 1$\left.)\right) ;$

$\%$ RY = calculate_RY ( phi $)$

$\mathrm{RZ}=$ calculate_RZ(phi); \%calculate rotation

$\%$ rotated_final $=$ rotated $* \mathrm{RY}$;

rotated_final $=$ rotated $* \mathrm{RZ}$; \%perform rotation

$\%$ Do one final check of magnitude and rotation to make sure we have the beam \%pointing where we want it to point

beam_mag $=$ beam_max $($ rotated_final $(:, 1)$, rotated_final $(:, 2), \ldots$

rotated_final $(:, 3))$;

[ beam_max_val beam_max_point] $=\max ($ beam_mag $)$;

rotated_final (beam_max_point, 1), rotated_final (beam_max_point ,2),.. rotated_final (beam_max_point, 3 )

$\%$ Save the rotated matrix in case of later issues or other needs save ('rot_final.mat',' rotated_final')

$\%$ Plot for Final Rotated Beam Pattern

$\%$ Uncomment this section to plot the final rotated beam pattern $\%\{$

figure ;

hold on;

plot3 ( rotated_final $(:, 1)$, rotated_final $(:, 2)$, rotated_final $(:, 3)$, , ); 
axis $\left(\left[\begin{array}{llllll}-1 & 1 & -1 & 1 & -1 & 1\end{array}\right]\right.$, 'equal' $)$;

$\%$ \}

$\%$ Find Beamwidth

\%Look at beam every $X$ degrees, create a slice of the beampattern, and then \%examine the slice of the beampattern to find the beamwidth

$\%$ set angle to rotate $\mathrm{X}$ degrees

angled $=0.5$;

angle $=\operatorname{deg} 2 \operatorname{rad}($ angled $)$;

rot_mat $=$ calculate RX $_{-}($angle $) ;$

current_pattern = rotated_final; \%Set first pattern to view

delta $=0.02$

lda $=180 /$ angled ;

valmat $=$ zeros $(361,1 \mathrm{da})$;

pg=figure ;

for $\mathrm{ii}=1: 180 /$ angled

\% grab a slice close to the XY plane clear slice;

[ slice kk] = get_XY_slice (current_pattern, delta);

if $\mathrm{i} i \mathrm{i}==1$ save ('slice.mat',', slice')

end

\%convert the slice to polar coordinates

[theta, mag $]=$ polar convert $_{-} \operatorname{slice}(:, 1)$, slice $(:, 2)$, slice $\left.(:, 3)\right)$;

$\operatorname{mag}_{-} \mathrm{db}=20 * \log 10(\mathrm{mag}) ;$

$\%$ figure ;

$\%$ polarplot (theta, mag_db,'. ') ;

$\% \operatorname{rlim}\left(\left[\begin{array}{ll}-30 & 0\end{array}\right]\right)$; 
\%now sort the slice in polar coordinates

[ theta ${ }_{-}$sorted, jdex] = sort (theta);

mag_db_sorted $=\operatorname{mag}_{-} \mathrm{db}(\mathrm{jdex}(:))$;

$[m, d e x]=\max \left(\operatorname{mag}_{-} \mathrm{db}_{-}\right.$sorted $) ;$

for $11=\operatorname{dex}: k \mathrm{k}$

if $\mathrm{mag}_{-} \mathrm{db}_{-}$sorted (11) $<=-3.0$

size $1=11$;

size $1 \mathrm{~m} 1=11-1$;

break;

end

end

for $11=\operatorname{dex}:-1: 0$

if $\mathrm{mag}_{-} \mathrm{db}_{-}$sorted (11) $<=-3.0$

size $2=11$;

$\operatorname{size} 2 \mathrm{~m} 1=11+1$

break;

end

end

\%linearly interpolate the location of the two $-3 \mathrm{~dB}$ points using \%circular coordinates - depreciated

right_mult $=\left(-3-\mathrm{mag}_{-} \mathrm{d}_{-}\right.$sorted $\left._{-}(\operatorname{size} 1 \mathrm{~m} 1)\right) / \ldots$

$\left(\operatorname{mag}_{-} d b_{-}\right.$sorted $(\operatorname{size} 1)-\mathrm{mag}_{-} \mathrm{db}_{-}$sorted $\left.(\operatorname{size} 1 \mathrm{~m} 1)\right)$;

right_point $=\left(\right.$ theta sorted $_{-}($size 1$)-$ theta sorted $\left._{-}(\operatorname{size} 1 \mathrm{~m} 1)\right) \ldots$

*right_mult + theta_sorted ( $\operatorname{size} 1 \mathrm{~m} 1)$;

left_mult $=\left(-3-\mathrm{mag}_{-} \mathrm{db}_{-} \mathrm{sorted}_{-}(\operatorname{size} 2 \mathrm{~m} 1)\right) / \ldots$

$\left(\mathrm{mag}_{-} \mathrm{db}_{-}\right.$sorted $(\operatorname{size} 2)-\mathrm{mag}_{-} \mathrm{db}_{-}$sorted $\left.(\operatorname{size} 2 \mathrm{~m} 1)\right)$;

left point $_{-}($theta sorted $(\operatorname{size} 2)-$ theta - sorted $(\operatorname{size} 2 \mathrm{~m} 1)) \ldots$

$*$ left_mult + theta_sorted (size $2 \mathrm{~m} 1)$;

beamwidth $=$ abs $($ right_point -1 eft_point $) /$ pi $* 180$ 
$\%$ Do Scattered Interpolate (comment out when not performing scattered $\%\{$ \%interpolation for entire set)

$$
\text { sy=s sice }(:, 2)
$$

[sys, Is ] = sort (abs ( sy));

$\operatorname{sxs}=\operatorname{sice}(:, 1)$;

sxs $=$ sx s ( Is );

szs=s lice $(:, 3)$;

$\mathrm{szs}=\mathrm{szs}$ ( Is );

sys $=$ sys $(1: 1000)$;

sXs $=\operatorname{sXs}(1: 1000)$;

$\mathrm{szs}=\mathrm{szs}(1: 1000)$; $\%\}$

[thetainterp, phiinterp, rhointerp] = cart2sph(sxs, sys, szs $)$;

$\%$ do linear Interpolate

$\%$ (Comment out when doing scattered Interpolation)

[ thetainterp, phiinterp, rhointerp] $=\operatorname{cart} 2 \operatorname{sph}(\operatorname{slice}(:, 1), \ldots$ slice $(:, 2)$, slice $(:, 3))$;

$\%$ Convert interpolated points

[theta0, phi0, rhointerp]=interpsph (thetainterp, phiinterp, rhointerp);

[xpl, ypl, zpl]=sph2cart (theta0, phi0, rhointerp);

$\mathrm{rdb}=20 * \log 10($ rhointerp); \%Convert gains to dB power

valmat $(:$, ii $)=$ rhointerp; \%create a $2 \mathrm{D}$ vector of the power values

$\%$ pg=figure ;

$\%$ Clear figure, plot current 2D beampattern, save frame for movie clf

hold on

plot ( rdb);

axis ( [ $\left.\left.\begin{array}{llll}0 & 361 & -40 & 0\end{array}\right]\right)$

Fm( i i , 1)=getframe ;

$\%$ close (pg) 
$\%$ pg $2=$ figure ;

\%Clear figure, plot current 3D slice, save for movie clf

plot3 ( slice $(:, 1)$, slice $(:, 2)$, slice $(:, 3)$, . ' ');

hold on

plot3 (xpl, ypl, zpl, '. $\left.\mathrm{r}^{\prime}\right)$;

ax is equal

view $(0,0)$

Fm( i i , 2)= getframe;

$\%$ close $($ pg2)

\%Look for the beam: Split beam pattern in half to look at the "low" values $\%$ (i.e. degree values less than 0) and high values (i.e. degree values $\%$ greater than 0) with 0 (point number 181 in the vector) being the MRA.

\%The distance from the MRA on both sides of the beamwidth are calculated to \%where they cross the $-3 \mathrm{~dB}$ threshold and then the beamdwidth is calucalted \%by adding both sides together.

rdblow $=$ rdb $(1: 181)$;

rdb3low $=$ rdblow $>=-3$;

rdb3lowdiff=abs ( diff $($ rdb3low $))$;

rdb3 lowdiffl=rdb3 lowdiff $==1$;

rdb3lowdiffnum=length (rdblow ( rdb3lowdiffl ));

if rdb3lowdiffnum == 1 \%o Side lobes

rdblows=rdblow $($ rdb3low $)$;

szlow = size ( rdblows );

lowdegadded $=((-3-\operatorname{rdb}(181-\operatorname{szlow}(1)+1)) /(\operatorname{rdb}(181-\operatorname{szlow}(1))-\ldots$

$\operatorname{rdb}(181-\operatorname{szlow}(1)+1)))$;

else \%Side lobes present, find just mainlobe

szlow $=0$;

$\mathrm{ik}=0$;

while ik $==0$

szlow $=$ s zlow +1 ;

$\mathrm{ik}=\mathrm{rdb} 31$ owdiffl $(181-\mathrm{szlow})$;

end

lowdegadded $=((-3-\operatorname{rdb}(181-\operatorname{szlow}(1)+1)) /(\operatorname{rdb}(181-\operatorname{szlow}(1))-\ldots$ 


$$
\operatorname{rdb}(181-\operatorname{szlow}(1)+1)))
$$

end

rdbhigh=rdb (181:361);

rdb3high=rdbhigh $>=-3$;

rdb3highdiff=abs ( diff (rdb3high));

rdb3highdiffl=rdb3highdiff $==1$;

rdb3highdiffnum=length (rdbhigh ( rdb3highdiffl));

if rdb3highdiffnum == 1 \%no Side lobes

rdbhighs=rdbhigh ( rdb3high);

szhigh=size ( rdbhighs);

highdegadded $=((-3-$ rdbhigh $(\operatorname{szhigh}(1))) /(\operatorname{rdbhigh}(\operatorname{szhigh}(1)+1)-\ldots$ rdbhigh ( szhigh (1))));

else \%Side lobes present, find just mainlobe szhigh $=0$;

$\mathrm{i} \mathrm{k}=0$;

while ik $==0$

szhigh=szhigh +1 ;

ik=rdb3highdiffl( szhigh);

end

highdegadded= $=((-3-$ rdbhigh $(\operatorname{szhigh}(1))) /(\operatorname{rdbhigh}(\operatorname{szhigh}(1)+1)-\ldots$ rdbhigh (szhigh (1))));

end

bwscatinterp=szlow (1)+szhigh (1)+lowdegadded+highdegadded -2 ;

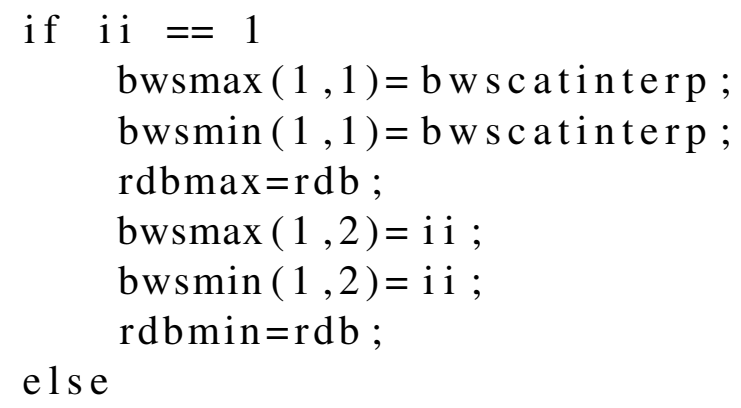




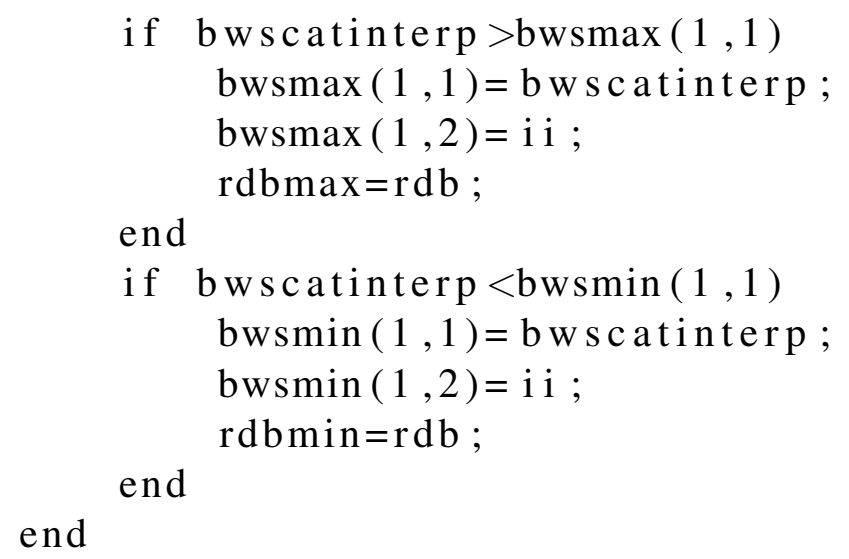

$\% \%$

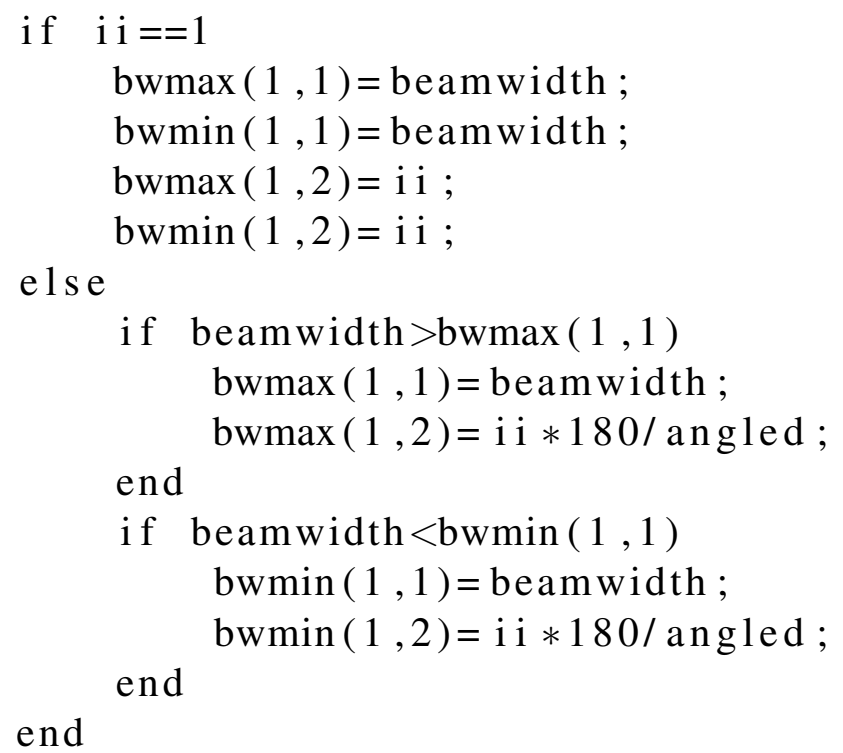

$\%$ CompMatrix ( ii , 1$)=$ beamwidth ;

$\%$ CompMatrix (ii, 2$)=$ bwscatinterp ;

$\%$ CompMatrix ( ii , 3) = beamwidth / bwscatinterp ;

temp $=$ current pattern;

current_pattern $=$ temp $*$ rot_mat;

$\%$ sprintf('Run \%d of \%d finished', ii, 180/angled) 
end

close (pg)

$\% \%$ Go back and find the min and max slice from polar coords, interpolate, \%and output beam patterns - Depreciated

\%This section is depreciated and not in use, uncomment if there is a $\%$ desire to use the polar coordinates

\%instead to calculate beamwidth

$\%\{$

angledmax $=\operatorname{bwmax}(1,2)$; \%Get Max BW Angle

angledmin $=\operatorname{bwmin}(1,2)$; \%Get Min BW Angle

anglemax $=\operatorname{deg} 2 \mathrm{rad}(\operatorname{angledmax})$;

anglemin $=\operatorname{deg} 2 \mathrm{rad}($ angledmin $)$;

rot_mat_max = calculate_RX (anglemax); \%Get the rotation matrix for max

rot_mat_min = calculate RX $_{-}($anglemin $) ; \%$ Get the rotation matrix for min

$\max _{-}$pattern $=$rotated_final $*$ rot_mat_max;

$\min _{-}$pattern $=$rotated_final $*$ rot_mat_min $_{-}$

\% grab a slice close to the XY plane

clear slice ;

[ slicemax kk] = get $X_{-} Y_{-}$slice $\left(\max _{-}\right.$pattern, delta $)$;

[ slicemin kk] = get $X_{-} Y_{-}$slice $\left(\min _{-}\right.$pattern, delta);

$\%$ Look at slices and interpolate

for $\mathrm{i}$ i $=1: 2$

if i i $==1$

slice $=$ slice $\max$;

elseif ii $==2$

slice =slicemin ;

end

sy= slice $(:, 2)$;

[sys, Is ] = sort $(\operatorname{abs}(\mathrm{sy}))$;

$\operatorname{sxs}=\operatorname{slice}(:, 1)$;

$\mathrm{sxs}=\operatorname{sxs}($ Is );

$\operatorname{szs}=\operatorname{slice}(:, 3)$;

$\mathrm{szs}=\mathrm{szs}($ Is ); 


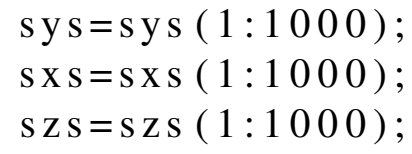

[thetainterp, phiinterp, rhointerp] = cart2sph(sxs, sys, szs $)$;

[theta0, phi0, rhointerp]=interpsph(thetainterp, phiinterp, rhointerp); $\mathrm{rdb}=20 * \log 10($ rhointerp $)$;

$\%$ This part commented out for generating movie frames, uncomment section if you want to create a move from the slices to view the progresion through the beam angles $\%\{$

hold on

$\mathrm{pg}=\mathrm{figure}$;

$\mathrm{plot}(\mathrm{rdb})$;

axis ( [ $\left.\left.\begin{array}{llll}0 & 361 & -40 & 0\end{array}\right]\right)$

Fm( i i ) = getframe;

close (pg)

$\%\}$

$\%\}$

$\%$ Movie: Create and show Movie

\%Uncomment this section to, after running the program, run the movie $\%$ created from the slices of the beampattern

$\%$ movie

\%movie (Fm)

$\%$ cla

\%movie (M) 
function $[\mathrm{mag}]=$ beam_max $(\mathrm{CX}, \mathrm{CY}, \mathrm{CZ})$

$\%$ Find the maximum of the beam from it's carteisian coordinates $\left[\begin{array}{ll}r & c\end{array}\right]=\operatorname{size}(\mathrm{CX})$;

for $\mathrm{j} j=1: \mathrm{r}$

for $\mathrm{ii}=1: \mathrm{c}$

$\operatorname{mag}(\mathrm{jj}, \mathrm{i} i)=\operatorname{sqrt}\left(\mathrm{CX}(\mathrm{jj}, \mathrm{i} i) \cdot{ }^{\wedge} 2+\mathrm{CY}(\mathrm{jj}, \mathrm{i} i) \cdot{ }^{\wedge} 2+\mathrm{CZ}(\mathrm{jj}, \mathrm{i} i) \cdot{ }^{\wedge} 2\right)$

end

end

function [theta, mag] = polar_convert $(\mathrm{X}, \mathrm{Y}, \mathrm{Z})$

$\%$ Convert the beam to poly coordinates from cartesian coordinates $[\mathrm{r}, \mathrm{c}]=\operatorname{size}(\mathrm{X})$;

for $\mathrm{j} j=1: \mathrm{r}$

$\operatorname{mag}(\mathrm{jj})=\operatorname{sqrt}\left(\mathrm{X}(\mathrm{jj}) \cdot{ }^{\wedge} 2+\mathrm{Y}(\mathrm{jj}) \cdot{ }^{\wedge} 2+\mathrm{Z}(\mathrm{jj}) \cdot{ }^{\wedge} 2\right)$;

theta $(\mathrm{jj})=\operatorname{atan} 2(\mathrm{Z}(\mathrm{j} \mathrm{j}), X(\mathrm{jj}))$;

end

function $[R]=$ calculate RX $_{-}$(theta)

$\%$ Calculate a rotation matrix around the $\mathrm{X}$-axis $\mathrm{R}=\left[\begin{array}{lll}1 & 0 & 0\end{array}\right.$

$0 \cos ($ theta $)-\sin ($ theta $)$

$0 \sin ($ theta $) \quad \cos ($ theta $)]$;

function $[R]=$ calculate ${ }_{-}$RY (theta)

$\%$ Calculate a rotation matrix around the $\mathrm{Y}$-axis

$\mathrm{R}=[\cos ($ theta $) 0$ sin (theta)

$0 \quad 1 \quad 0$

$-\sin ($ theta) $0 \cos ($ theta $)]$

function $[R]=$ calculate $R_{-} R($ theta $)$ 
$\%$ Calculate a rotation matrix around the $\mathrm{Z}$-axis

$$
\mathrm{R}=[\cos (\text { theta })-\sin (\text { theta }) \quad 0
$$

$\sin ($ theta $) \quad \cos ($ theta $) \quad 0$

$0 \quad 0 \quad 1]$;

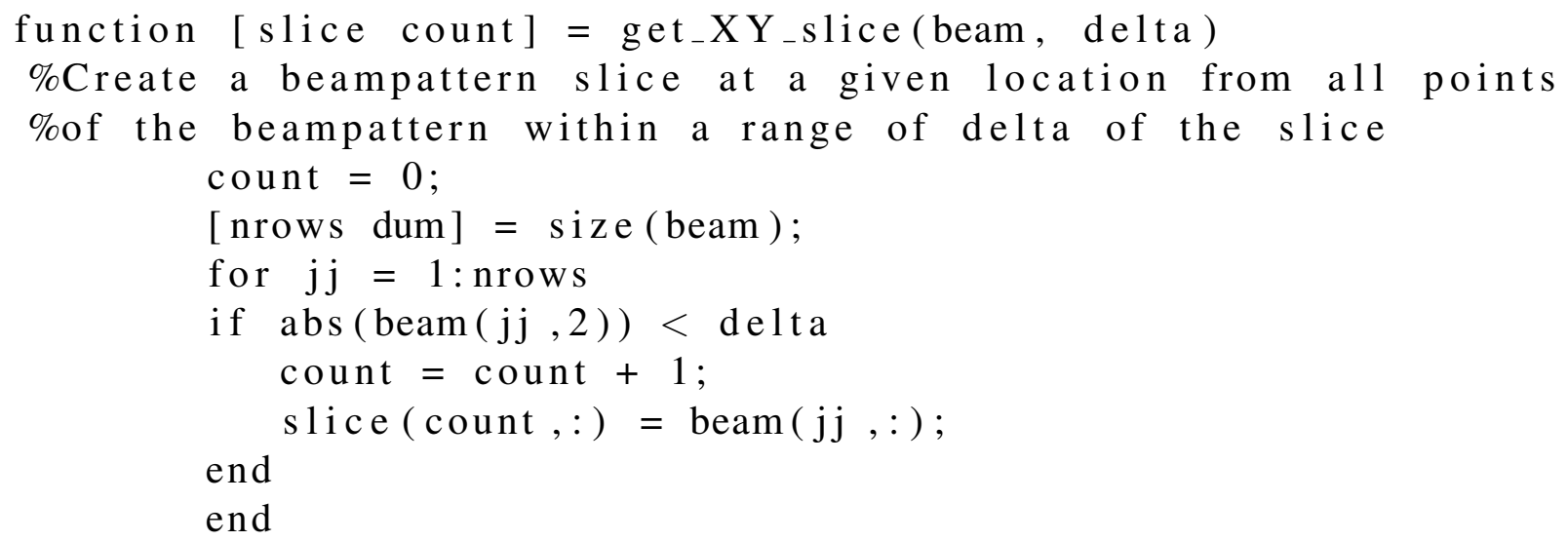

\section{Appendix A.3: Beampattern Post Processing Code}

\%This progarm takes the values previously calculated - Geometry and \%beampattern slices - and then finds additonal information in terms of $-3 \mathrm{~dB}$ \%beamwidth including sidelobes and $-6 \mathrm{~dB}$ beamwidth both including and \%excluding sidelobes

$\%$ Load in variables load vstruct.mat \%Load in the structure of information load strdone.mat \%Load in the list of files which need this work done

$\% \%$ Process the code

lstrd=length (strd); \%Find the number of items that need to be done

for $\mathrm{i} i=1: 1 \mathrm{strd}$

namestr $=\operatorname{strd}\{\mathrm{i} i\}$; \%rab the item being done this iteration for $\mathrm{jj}=1: 10 \%$ Perform the following over all 10 recorded frequencies $\mathrm{sn}=\mathrm{num} 2 \mathrm{str}(\mathrm{jj})$;

\%Create a field name for the current frequency freqstr=strcat (' freq', sn ); 
$\%$ Get the interpolated values for this data set intv=valstruct. ( namestr). ( freqstr). interpvalues; bw3maxi $=0$; \%Preallocate Max BW between runs bw 3 mini $=1000$; \% Preallocate Min BW Between Runs bw6maxi $=0 ; \%$ Preallocate Max BW between runs bw6mini $=1000$; \% Preallocate Min BW Between Runs bw6maxo=0; \%Preallocate Max BW between runs bw6mino $=1000 ; \%$ Preallocate Min BW Between Runs mlwidthmin $=1000$; \% Preallocate mainlobe width Between Runs mlwidthmax $=0$; \% Preallocate Main Lobe Width between runs null1valhighmlmax $=0 ; \%$ Preallocate Null Value between runs null1vallowmlmax $=0$; \% Preallocate Null Value between runs null1valhighmlmin $=0 ; \%$ Preallocate Null Value between runs null1vallowmlmin $=0 ; \%$ Preallocate Null Value between runs

sl1 maxhigh $=0$;

s11 maxlow $=0$;

for $\mathrm{kk}=1: 180$

$\%$ Do this over 180 degrees (Assuming 1 degree slices, $\%$ change as needed)

intvtemp=intv (:, kk); \%Get data set for this run $\mathrm{rdb}=20 * \log 10($ intvtemp $)$;

$\%$ First find the $-3 \mathrm{db}$ and $-6 \mathrm{~dB}$ downpoints including sidelobes $\%$ Find lower point values rdblow=rdb (1:181); \%Get lower half of data set intvtemplow=intvtemp (1:181);\% Get lower half of data set rdb3low $=$ rdblow $>=-3 ; \%$ Threshold to $-3 \mathrm{~dB}$ rdb6low $=$ rdblow $>=-6 ; \%$ Threshold to $-6 \mathrm{~dB}$ rdb3lowdiff=diff ( rdb3low); \%Create Logical to threshold rdb6lowdiff =diff ( rdb6low); \%Create logical to threshold in $310 \mathrm{w}=0$; \% Reallocate for each run in $610 w=0 ; \%$ Reallocate for each run out6low $=0 ; \%$ Reallocate for each run run $=0 ; \%$ Reallocate for each run val $=0 ; \%$ Reallocate for each run $\%$ Find first spot where $-3 \mathrm{dBBW}$ goes over threshold from \%periphery (counting sidelobes) 


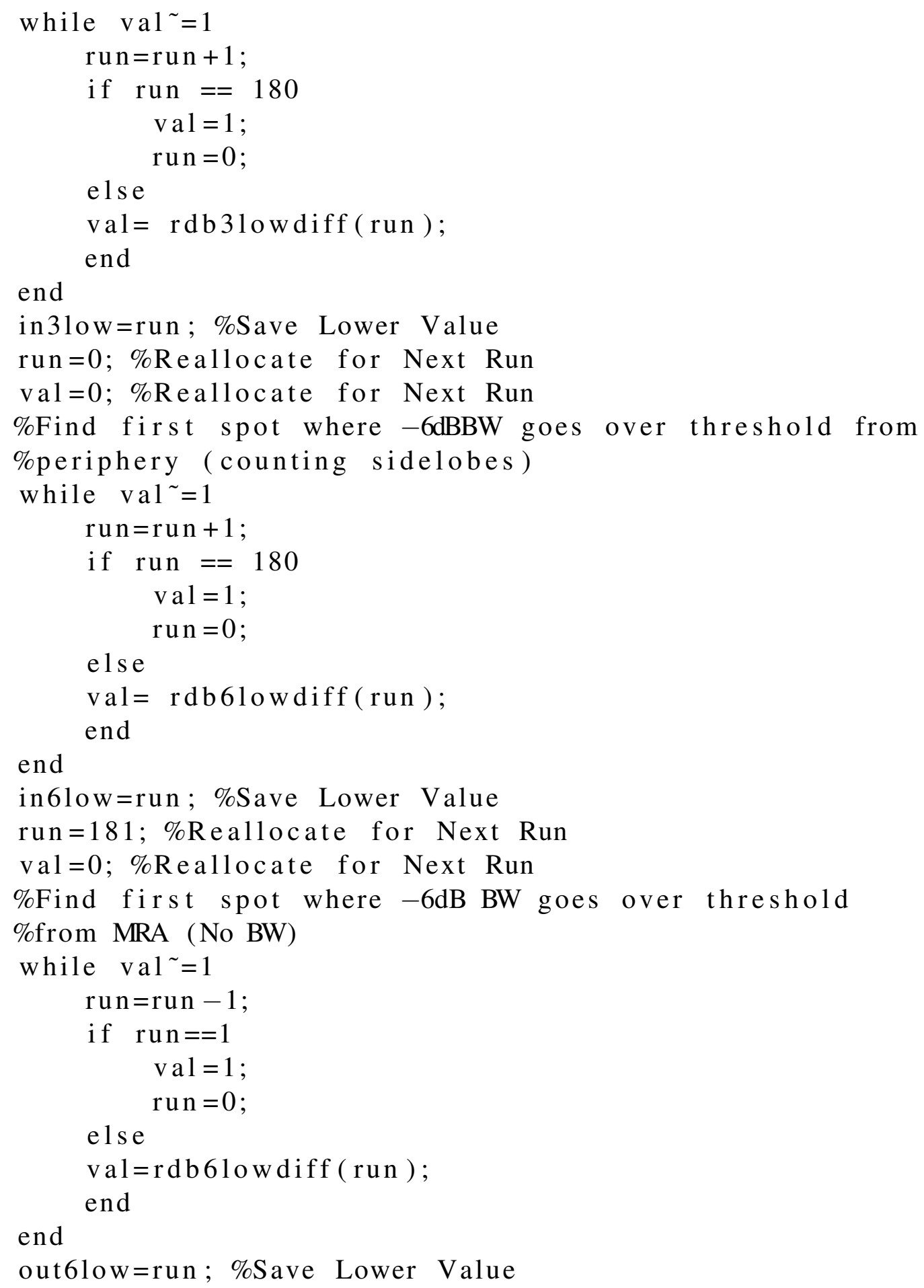


\%Look for max sidelobe values

[MaximaLow, MaximaLocLow]=findpeaks (intvtemplow);

\%There is no bottom peak (Values contstantly decreasing)

$\%$ set to edge

if isempty (MaximaLow)

sl1 maxlowvaltemp=intvtemplow (1);

sl1 maxlowloctemp $=1$;

else

$\%$ Set max value of side lobe

sl1 maxlowvaltemp=MaximaLow ( length (MaximaLow ));

$\%$ Set position of side lobe

sl1 maxlowloctemp=MaximaLocLow ( length (MaximaLocLow )); end

\%Invert to find min sidelobes

invintvtemplow $=1.01 * \max ($ intvtemplow $)$ - intvtemplow ;

$\%$ Find min sidelobes

[MinimaLow, MinimaLocLow]=findpeaks (invintvtemplow);

if isempty (MinimaLow) \%There were no nulls, set to edge

null1vallowtemp=intvtemplow (1);

mlwidthlow te $\mathrm{mp}=1$;

else

$\%$ Set the first null value

null1 vallow te mp=MinimaLow ( length (MinimaLow ) );

mlwidthlowtemp=MinimaLocLow $($ length $($ MinimaLocLow $))$;

end

$\%$ Find Higher Point Values

\%This section functions identically to the above section,

\%except all of the values and calculations are done for the

\%upper point values.

intvtemphigh=intvtemp (181:361); \%Take High values

rdbhigh=rdb(181:361); \%Get high dB values

rdb3high=rdbhigh $>=-3$; \% Threshold values

rdb6high=rdbhigh $>=-6 ; \%$ Threshold values

rdb3highdiff=diff(rdb3high); \%Create difference logical matrix rdb6highdiff=diff(rdb6high); \%Create difference logical matrix in 3 high $=0$; \% Preallocate Value for each run in6high $=0$; \% Preallocate Value for each run 


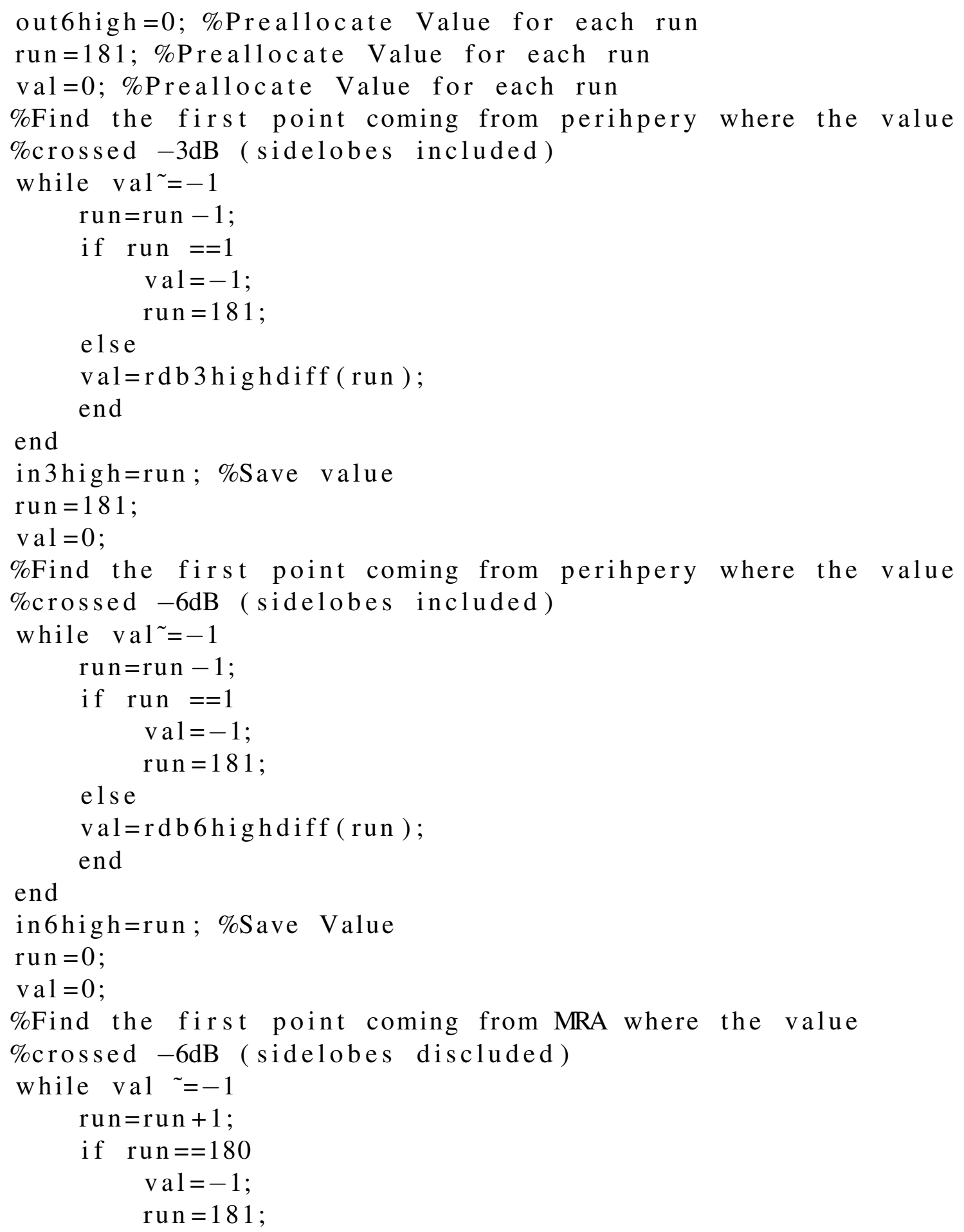




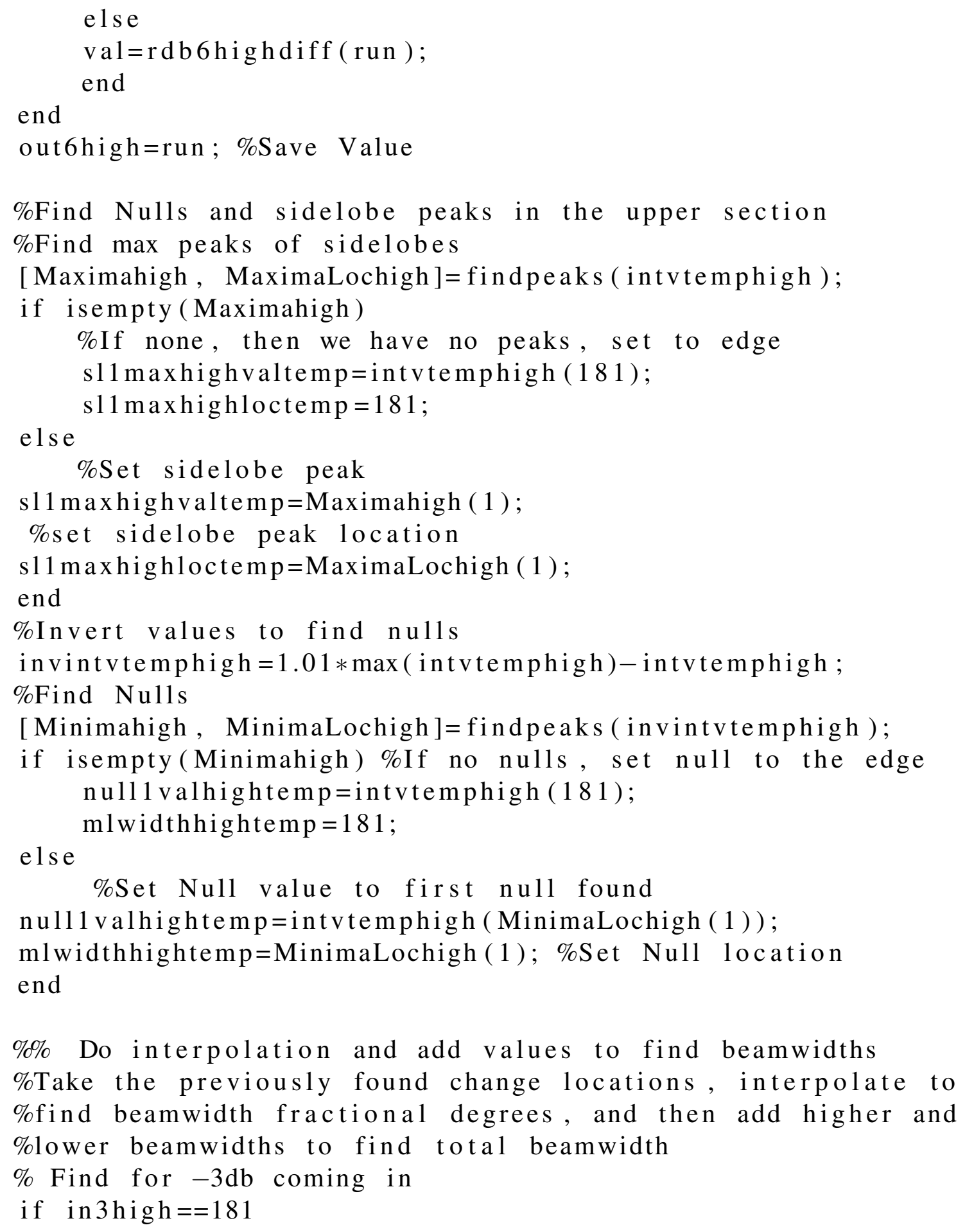




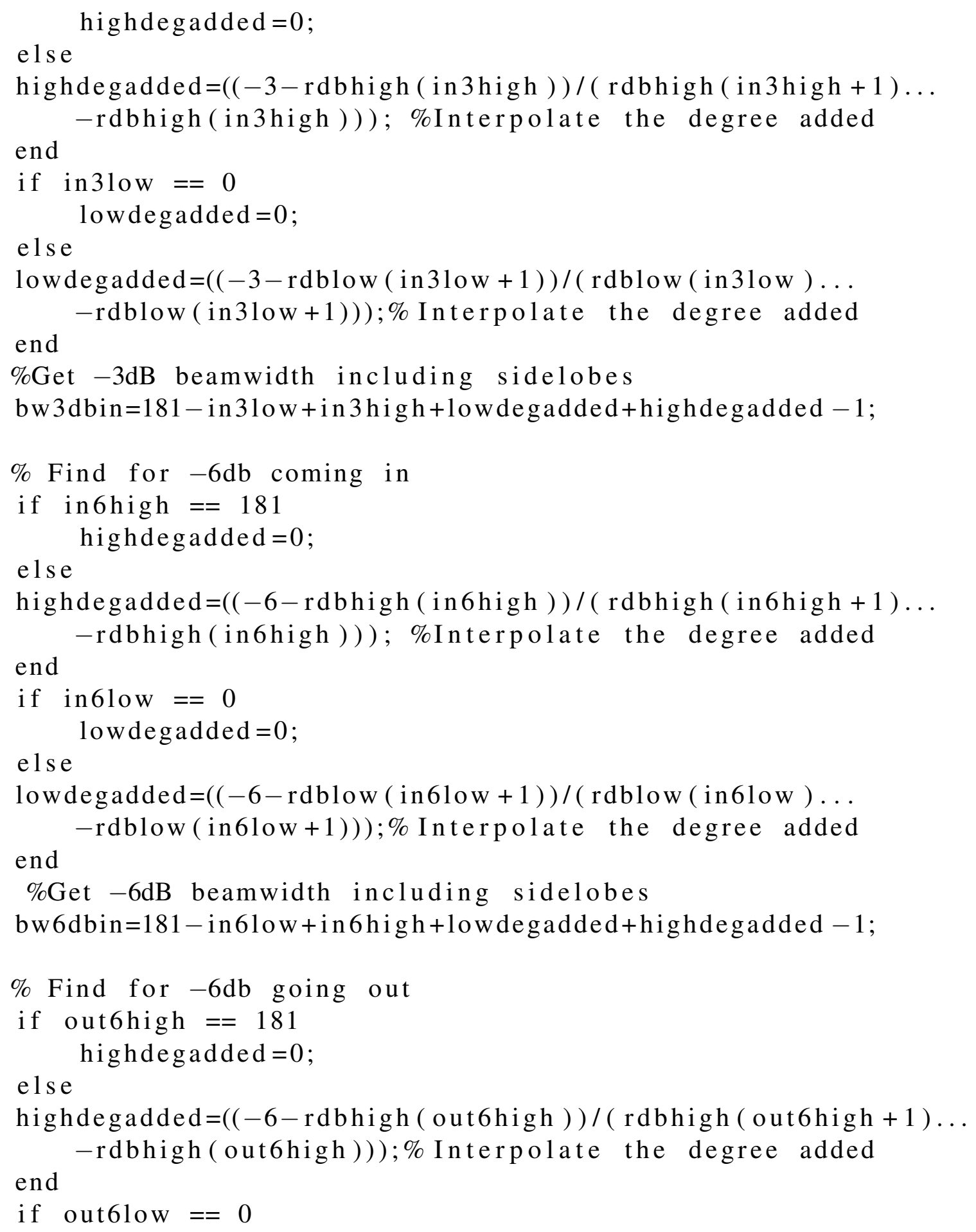


low degadded $=0$;

else

lowdegadded $=((-6-$ rdblow $($ out6low +1$)) /($ rdblow $($ out6low $) \ldots$

- rdblow $($ out6low +1$))) ; \%$ Interpolate the degree added end

\%Get $-6 \mathrm{~dB}$ beamwidth discluding sidelobes

bw 6 dbout $=181-$ out6low +out 6 high +lowdegadded +highdegadded -1 ;

\% Conditionals to see if we have new $\min / \max$ values

if bw 3 dbin $>$ bw 3 maxi \%Check to see if this is the new max bw 3 maxi=bw $3 \mathrm{dbin}$;

end

if bw3dbin <bw3mini \%Check to see if this is the new min bw 3 mini=bw 3 dbin ;

end

if bw6dbin $>$ bw6maxi \%Check to see if this is the new max bw6maxi=bw6 dbin;

end

if bw6dbin < bw6mini \%Check to see if this is the new min bw6mini=bw6 dbin ;

end

if bw6dbout $>$ bw6maxo \%Check to see if this is the new max bw6maxo=bw6dbout;

end

if bw6dbout $<$ bw6mino \%Check to see if this is the new min bw 6 mino=bw6 dbout;

end

$\%$ compute main lobe width and determine if max or min main lobe mlbwtemp $=$ mlwidthhighte $\mathrm{mp}-1+181-$ mlwidthlowte $\mathrm{mp}$;

if mlbwtemp $>$ mlwidthmax

mlwidthmax $=$ mlbwtemp ;

null1valhighmlmax =null1valhightemp ;

null1vallowmlmax $=$ null1 vallow te mp ;

end

if mlbwtemp $<$ mlwidthmin

mlwidthmin=mlbwtemp ; 
null1valhighmlmin=null1valhightemp ;

null1 vallowmlmin=null1 vallow temp ;

end

$\%$ Determine whether or not this is the largest side lobe

if sl1maxhighvaltemp >sl1maxhigh

sl1 maxhigh=sl1 maxhighvaltemp ;

end

if sl1maxlowvaltemp $>$ sl1maxlow

sl1 maxlow =sl1 maxlowvaltemp ;

end

$\%$ Move on to the next slice

end

$\%$ Find out if there were side lobes and save the $3 \mathrm{db}$ and $6 \mathrm{db}$

$\% \min / \max$ beamwidths

$\%$ Get $-3 \mathrm{~dB}$ max beamwidth with no sidelobes

bwsmax =valstruct . ( namestr $)$. ( freqstr ). bwsmax;

bw $3 \operatorname{maxo}=$ bwsmax $(1)$;

$\%$ Get $-3 \mathrm{~dB}$ min beamwidth with no sidelobes

bwsmin $=$ valstruct . ( namestr $)$. ( freqstr ). bwsmin;

bw $3 \operatorname{mino}=$ bwsmin $(1)$;

rdbmin $=$ valstruct $.($ namestr $) .($ freqstr $) . r d b \min$;

rdbmax $=$ valstruct $.($ namestr $) .($ freqstr $)$. rdbmax;

$\%$ Get interp values

valmat $=$ valstruct $.($ namestr $) .($ freqstr $)$. interpvalues ;

$\%$ See if they are the same or different including and

\%exlcuding sidelobes

if bw $3 \operatorname{maxo}==b w 3 \operatorname{maxi} \& \&$ bw $3 \operatorname{mino}==b w 3 \operatorname{mini}$

s13 $=0 ; \%$ No $3 \mathrm{~dB}$ sidelobes

els e

s13 $=1 ; \% 3 \mathrm{~dB}$ sidelobes

end

$\%$ See if they are the same or different including and \%exlcuding sidelobes 


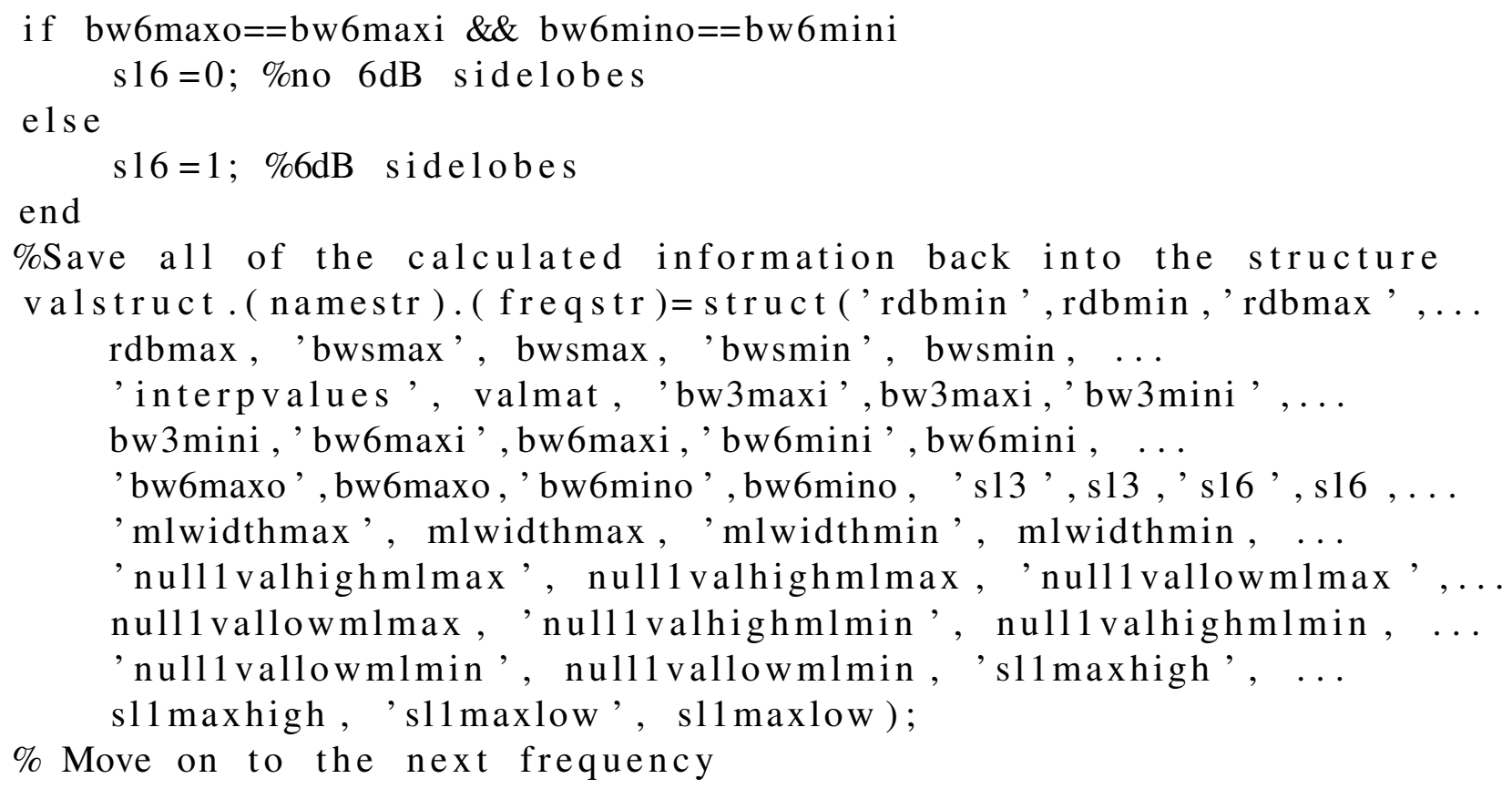


Appendix B: Raw Data Table 


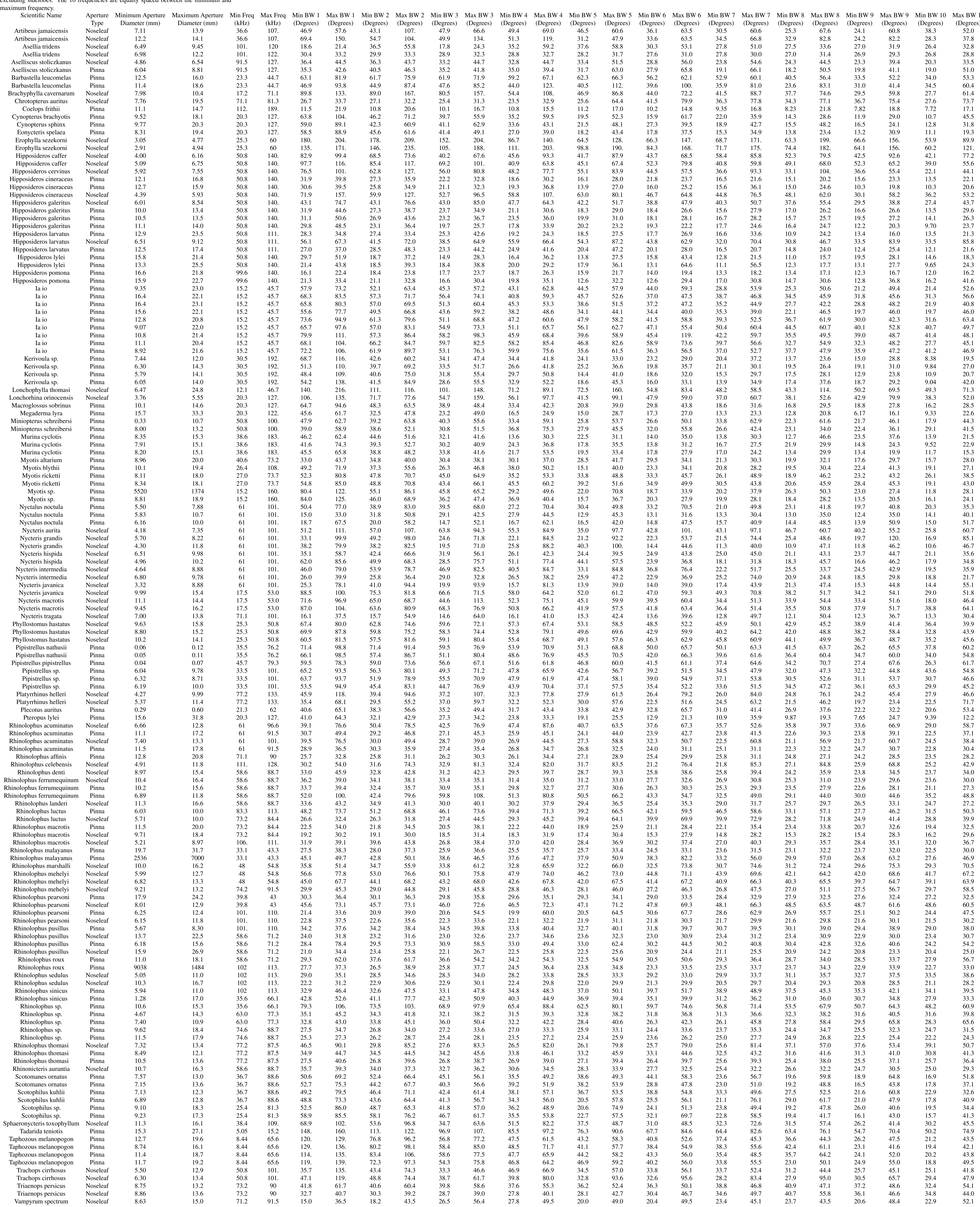


